\title{
Two-Electron Oxidative Atom Transfer at a Homoleptic, Tetravalent Uranium Complex
}

Natalie T. Rice,${ }^{\dagger}$ Karl McCabe,${ }^{\ddagger}$ John Bacsa,${ }^{\dagger}$ Laurent Maron, ${ }^{\ddagger}$ and Henry S. La Pierre ${ }^{\dagger, \# *}$

† School of Chemistry and Biochemistry, Georgia Institute of Technology, Atlanta, Georgia 30332-0400, United States.

‡ Laboratoire de Physique et Chimie des Nano-objets, Institut National des Sciences Appliquees, 31077 Toulouse, Cedex 4, France.

\# Nuclear and Radiological Engineering and Medical Physics Program, School of Mechanical

Engineering, Georgia Institute of Technology, Atlanta, Georgia 30332-0400, United States.

\section{Table of Contents}

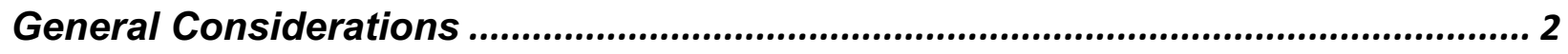

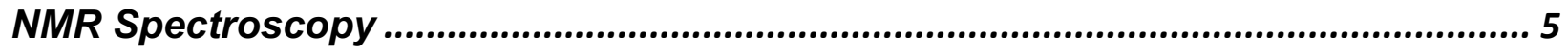

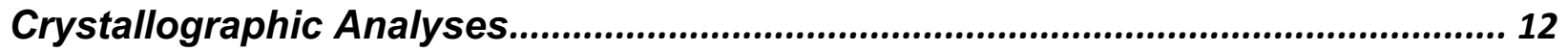

UV/vis/NIR electronic absorption spectra .................................................................. 28

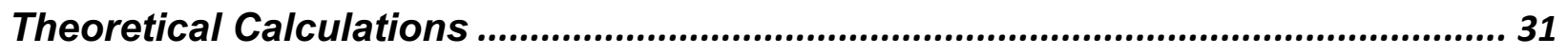

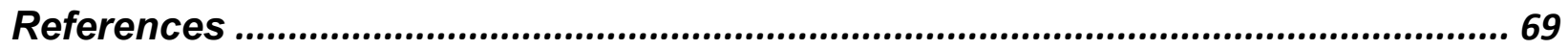




\section{General Considerations}

Unless otherwise noted, all reagents were obtained from commercial suppliers and the syntheses and manipulations were conducted under argon with exclusion of oxygen and water using Schlenk techniques or in an inert atmosphere box (Vigor) under a dinitrogen $\left(<0.1 \mathrm{ppm} \mathrm{O}_{2} / \mathrm{H}_{2} \mathrm{O}\right)$ atmosphere. The glovebox is equipped with two $-35{ }^{\circ} \mathrm{C}$ freezers. All glassware and cannulae were stored in an oven overnight $(>8 \mathrm{~h})$ at a temperature of ca. $160{ }^{\circ} \mathrm{C}$. Celite and molecular sieves were dried under vacuum at a temperature $>250{ }^{\circ} \mathrm{C}$ for a minimum of 24 h. $\mathrm{C}_{6} \mathrm{D}_{6}$ was stored over $3 \AA$ molecular sieves and then vacuum-transferred from purple sodium/benzophenone prior to use. Diethyl ether, $n$-pentane, $n$-hexane, benzene, toluene, tetrahydrofuran, and 1,2-dimethoxyethane were purged with UHP-grade argon (Airgas) and passed through columns containing Q5 and molecular sieves in a solvent purification system (JC Meyer Solvent Systems). All solvents in the glovebox were stored in bottles over $3 \AA$ molecular sieves. Methanol was dried by refluxing over magnesium turnings activated with iodine for $12 \mathrm{~h}$ and then distilled and stored over $3 \AA$ molecular sieves.

The starting materials $\mathrm{Ul}_{4}$ (dioxane $)_{2}$, [(piperidinyl $)_{3} \mathrm{PNK}$ ], and potassium benzyl were prepared according to literature procedures. ${ }^{1-3}$ Potassium $t$-butoxide was sublimed prior to use. NMR spectra were obtained on a Bruker Advance III $400 \mathrm{MHz}$ spectrometer at $298 \mathrm{~K}$, unless otherwise noted. ${ }^{1} \mathrm{H},{ }^{13} \mathrm{C}$, and ${ }^{31} \mathrm{P}$ NMR chemical shifts are reported in $\delta$, parts per million. ${ }^{1} \mathrm{H}$ NMR are referenced to the residual ${ }^{1} \mathrm{H}$ resonances of the solvent. ${ }^{13} \mathrm{C}$ NMR are referenced to the ${ }^{13} \mathrm{C}$ resonance of the deuterated solvent. ${ }^{4}$ Peak position is listed, followed by peak multiplicity, integration value, and proton assignment, where applicable. Multiplicity and shape are indicated by the following abbreviations: s (singlet); $\mathrm{d}$ (doublet); t (triplet); q (quartet); dd (doublet of doublets); td (triplet of doublets); $\mathrm{m}$ (multiplet); br (broad). Infrared (IR) samples were taken on a Bruker ALPHA FTIR spectrometer from 400 to $4000 \mathrm{~cm}^{-1}$. IR samples were prepared as Nujol mulls sandwiched between two $\mathrm{KBr}$ plates. The peaks are listed in wavenumber $\left[\mathrm{cm}^{-1}\right]$ and intensity using the following abbreviations: vw (very weak); w (weak); m (medium); s (strong); vs (very strong); br (broad). UV/visible/NIR spectroscopy was performed in Teflon-valve sealed quartz cuvettes with a $1 \mathrm{~cm}$ path length on a Hitachi UH4150 UV-visNIR scanning spectrophotometer between 2400-200 nm. Elemental analyses were determined at University of California, Berkeley, Microanalytical Facility (Berkeley, CA). 


\section{Synthetic Methods}

[U(pip) $\left.\left.{ }_{3} \mathbf{P N}\right)_{4}\right],(1-\mathbf{U}(\mathbf{P N}))$. Inside a glovebox, $4 \mathrm{~mL}$ diethyl ether was added to a $20 \mathrm{~mL}$ scintillation vial charged with $\mathrm{Ul}_{4}$ (dioxane $)_{2}(0.130 \mathrm{~g}, 0.141 \mathrm{mmol})$ and a glass stir bar. $\mathrm{K}\left[\mathrm{NP}\left(\mathrm{pip}_{3}\right](0.2 \mathrm{~g}, 0.579 \mathrm{mmol})\right.$ was added to the scintillation vial as a solid. An immediate color change to a pink suspension was observed. The reaction mixture was stirred overnight and then was filtered through a fine porosity frit with minimal additional diethyl ether. The remaining solid on the frit was washed with $15 \mathrm{~mL}$ toluene while crushing with a spatula. The toluene filtrate was concentrated in vacuo to afford the title compound as a pink crystalline solid in $94 \%$ yield $(0.157 \mathrm{~g}) .{ }^{1} \mathrm{H}$ NMR $\left(400.13 \mathrm{MHz}, \mathrm{C}_{6} \mathrm{D}_{6}\right): \delta 3.47(\mathrm{~s}, 48$ $\mathrm{H}), 1.03$ (s, $72 \mathrm{H}) .{ }^{31} \mathrm{P}$ NMR (161.98 MHz, $\left.\mathrm{C}_{6} \mathrm{D}_{6}\right): \delta 340.27$ (s). ${ }^{13} \mathrm{C} N M R(100.61 \mathrm{MHz}$, $\left.\mathrm{C}_{6} \mathrm{D}_{6}\right): \delta 28.04(\mathrm{~s}), 24.94(\mathrm{~s}), 24.66(\mathrm{~s})$. IR of $1-\mathrm{U}(\mathrm{PN})$ could not be obtained as the complex decomposes in nujol. Elemental analysis found(calculated) for: C 50.55(50.48), $\mathrm{H}$ 8.71(8.47), N 15.83(15.70). XRD quality crystals were grown from the diffusion of a solution of $1-\mathrm{U}(\mathrm{PN})$ in hexanes into paratone oil at room temperature.

[U(pip) $\left.{ }_{3} \mathrm{PN}\right)_{4}$ NMes], (2-U(PN)NMes). Inside a glovebox, $3 \mathrm{~mL}$ diethyl ether was added to a $20 \mathrm{~mL}$ scintillation vial charged with $1-\mathrm{U}(\mathrm{PN})(0.106 \mathrm{~g}, 0.0742 \mathrm{mmol})$ and a Teflon stir bar. Mesityl azide $(0.012 \mathrm{~g}, 0.0742 \mathrm{mmol})$ was added to the scintillation vial as a solution in diethyl ether $(1 \mathrm{~mL})$ and the reaction mixture was stirred for $1 \mathrm{hr}$. An immediate color change from a pink suspension to a black solution was observed. The mixture was filtered through a pipet filter packed with Celite and glass filter paper to give a black solution which was concentrated in vacuo and placed into a $-35{ }^{\circ} \mathrm{C}$ freezer. Overnight a black microcrystalline solid formed which was isolated by decantation and the residual volatiles were removed in vacuo to afford the title compound $(82 \%, 0.094 \mathrm{~g}) .{ }^{1} \mathrm{H}$ NMR $(400.13$ $\left.\mathrm{MHz}, \mathrm{C}_{6} \mathrm{D}_{6}\right): \delta 8.46(\mathrm{~s}, 2 \mathrm{H}), 6.00(\mathrm{~s}, 3 \mathrm{H}), 5.46(\mathrm{~s}, 6 \mathrm{H}), 3.46(\mathrm{~s}, 48 \mathrm{H}), 1.60(\mathrm{~s}, 72 \mathrm{H}) .{ }^{31} \mathrm{P}$ NMR (161.98 MHz, $\left.\mathrm{C}_{6} \mathrm{D}_{6}\right): \delta 2.07$ (s). ${ }^{13} \mathrm{C} \mathrm{NMR}\left(100.61 \mathrm{MHz}, \mathrm{C}_{6} \mathrm{D}_{6}\right): \delta 181.71$ (s), 150.13 (s), 109.33 (s), 46.85 (s), 27.69 (d), 25.90 (s), 15.59 (s), 12.17 (s), 10.85 (s). IR: $v$ [cm $\left.{ }^{-1}\right]$ $=1332(\mathrm{w}), 1326(\mathrm{w}), 1272(\mathrm{w}), 1260(\mathrm{w}), 1216(\mathrm{~m}), 1204(\mathrm{~m}), 1155(\mathrm{~m}), 1120(\mathrm{vs}), 1091$ (vs), 1058 (vs), 1023 (s), 949 (s), $852(\mathrm{~m}), 836(\mathrm{w}), 811(\mathrm{w}), 667$ (w). Elemental analysis found(calculated) for: C 52.87(53.10), H 8.42(8.46), N 15.25(15.26). XRD quality crystals were grown from a concentrated solution of $2-\mathrm{U}(\mathrm{PN}) \mathrm{NMes}$ in $n$-pentane at $-35^{\circ} \mathrm{C}$.

[U $\left.\mathrm{U}^{6+}\left(\text { pip) }{ }_{3} \mathrm{PN}\right)_{4} \mathrm{NO}\right],(2-\mathrm{U}(\mathrm{PN}) \mathbf{O})$. Inside a glovebox, $5 \mathrm{~mL}$ THF was added to a $20 \mathrm{~mL}$ scintillation vial charged with 1-U(PN), $(0.160 \mathrm{~g}, 0.112 \mathrm{mmol})$ and a Teflon stir bar. A balloon of $\mathrm{N}_{2} \mathrm{O}$ was affixed to the scintillation vial and the reaction mixture was allowed to stir for $1 \mathrm{~h}$. During this time, the pink suspension became a dark red solution. Volatiles were removed in vacuo and the residue was triturated two times with $2 \mathrm{~mL} n$-pentane. The residue was then taken up in $25 \mathrm{~mL}$ of n-pentane and filtered through a pipet filter packed with Celite and glass filter paper to give a deep red solution which was concentrated in vacuo and placed into a $-35^{\circ} \mathrm{C}$ freezer. Overnight a red microcrystalline solid formed which was isolated by decantation and residual volatiles were removed in vacuo to afford the title compound $(84 \%, 0.137 \mathrm{~g}) .{ }^{1} \mathrm{H}$ NMR $\left(400.13 \mathrm{MHz}, \mathrm{C}_{6} \mathrm{D}_{6}\right): \delta 3.45$ $(\mathrm{s}, 48 \mathrm{H}), 1.62$ (s, $72 \mathrm{H}) .{ }^{31} \mathrm{P}$ NMR (161.98 MHz, $\left.\mathrm{C}_{6} \mathrm{D}_{6}\right): \delta 5.81$ (s). ${ }^{13} \mathrm{C}$ NMR $(100.61 \mathrm{MHz}$, $\left.\mathrm{C}_{6} \mathrm{D}_{6}\right): \delta 46.78(\mathrm{~s}), 27.72\left(\mathrm{~d}, 26.08(\mathrm{~s}) . \mathrm{IR}: v\left[\mathrm{~cm}^{-1}\right]=1354(\mathrm{w}), 1332(\mathrm{~m}), 1326(\mathrm{~m}), 1274\right.$ (w), 1256 (w), $1216(\mathrm{~s}), 1202(\mathrm{~m}), 1159(\mathrm{~m}), 1122$ (vs), 1103 (vs), 1058 (s), 1025 (s), 949 
(s), $854(\mathrm{~m}), 834(\mathrm{w}), 811(\mathrm{w}), 760(\mathrm{w}), 669(\mathrm{vw})$. Elemental analysis found(calculated) for: C 49.91(49.92), H 8.40(8.38), N 15.49(15.52). XRD quality crystals were grown from diffusion of a solution of $2-\mathrm{U}(\mathrm{PN}) \mathrm{O}$ diethyl ether into paratone oil at $-35^{\circ} \mathrm{C}$. 


\section{NMR Spectroscopy}

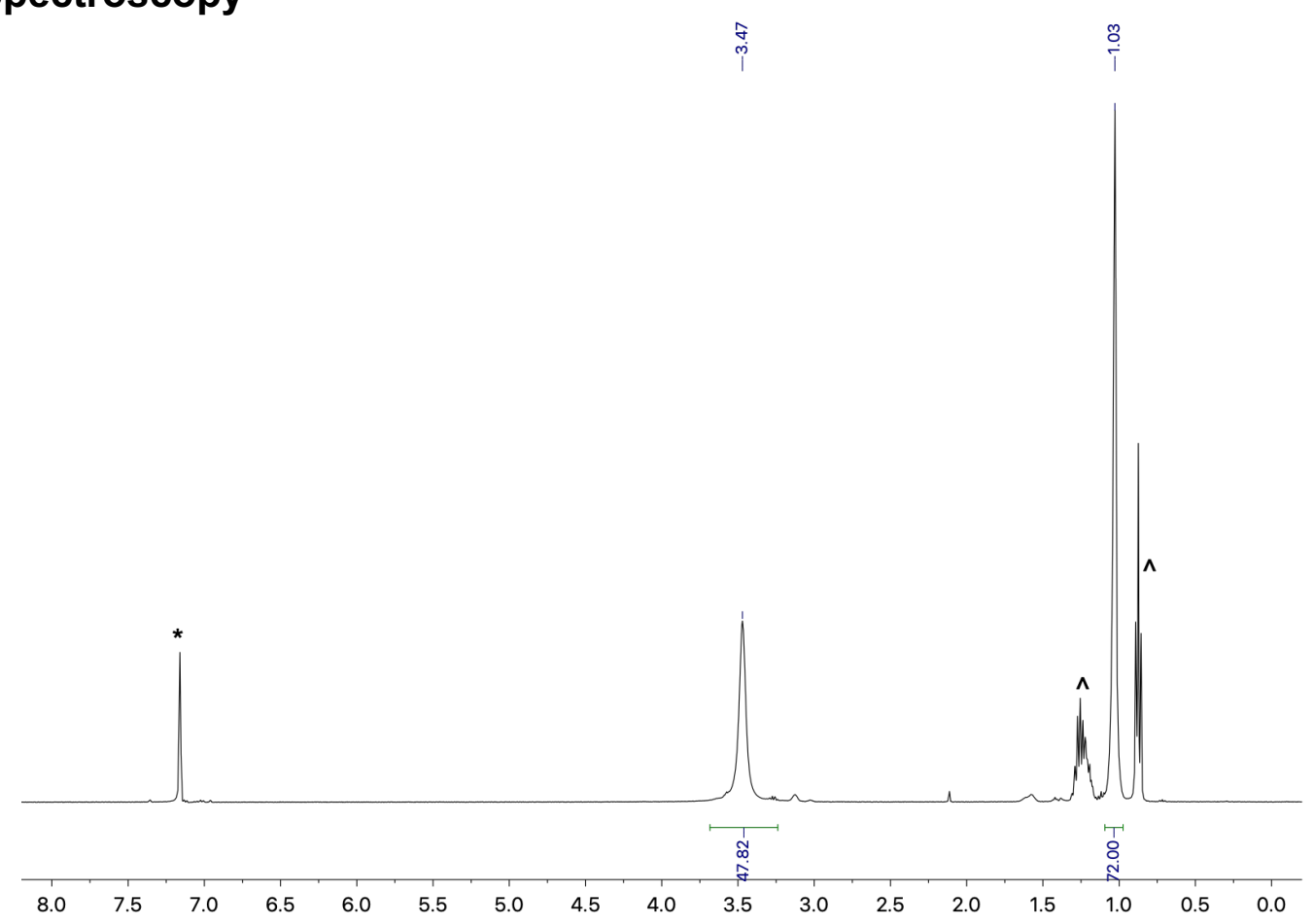

Figure S1. ${ }^{1} \mathrm{H}$ NMR of $1-\mathrm{U}(\mathrm{PN})$ in $\mathrm{C}_{6} \mathrm{D}_{6}$. Peak of $\mathrm{C}_{6} \mathrm{D}_{5} \mathrm{H}$ is noted as *. Residual $n$ pentane is denoted by ${ }^{\wedge}$.

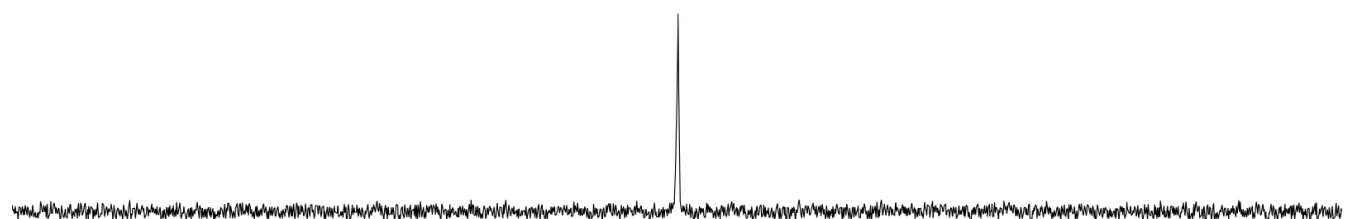

Figure S2. ${ }^{31} \mathrm{P}\left\{{ }^{1} \mathrm{H}\right\} \mathrm{NMR}$ of $1-\mathrm{U}(\mathrm{PN})$ in $\mathrm{C}_{6} \mathrm{D}_{6}$. 


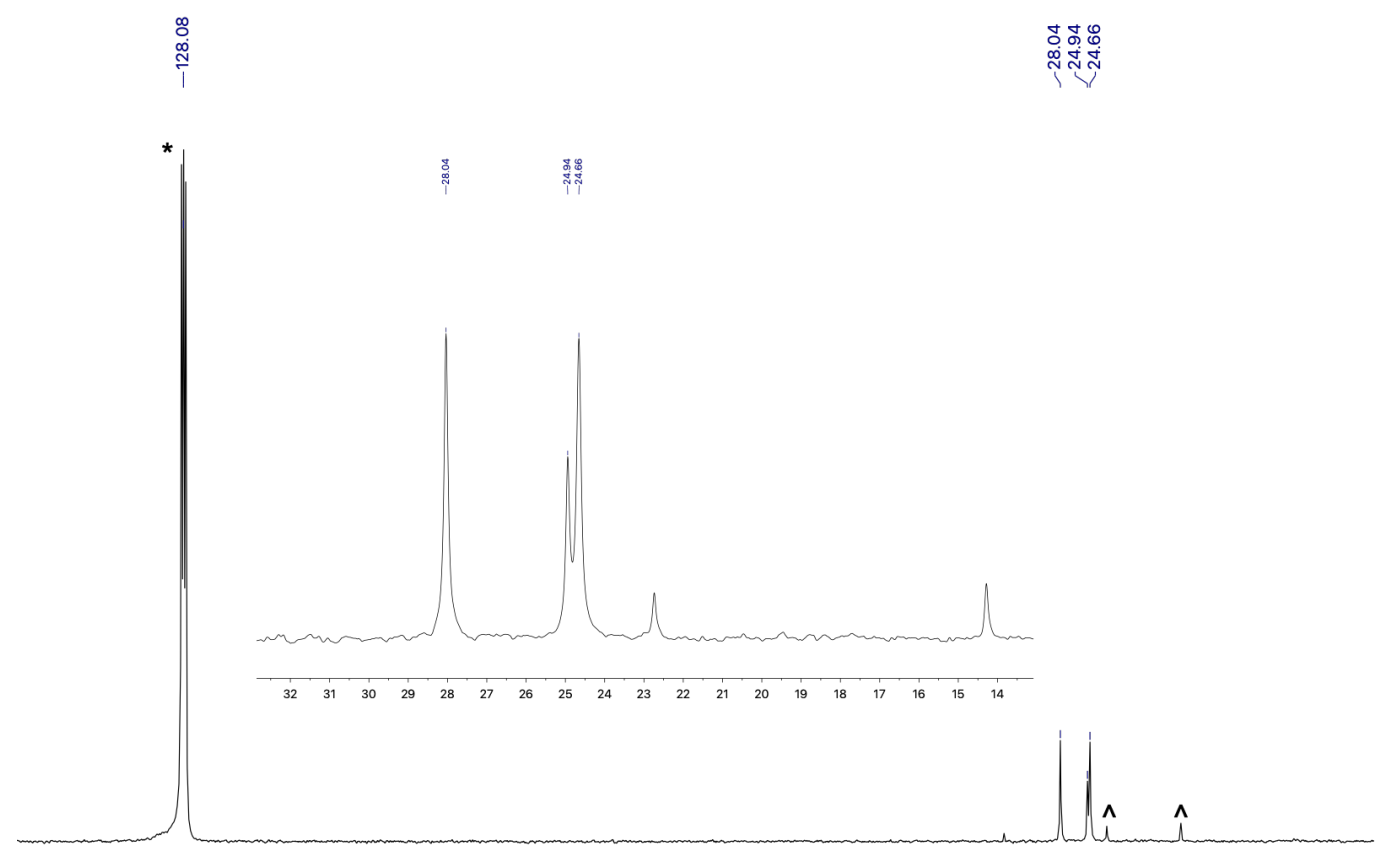

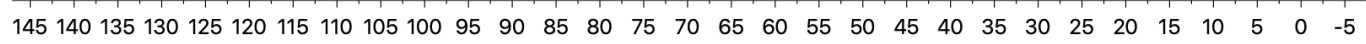

Figure S3. ${ }^{13} \mathrm{C}\left\{{ }^{1} \mathrm{H}\right\}$ NMR of $1-\mathrm{U}(\mathrm{PN})$ in $\mathrm{C}_{6} \mathrm{D}_{6}$. Peak of $\mathrm{C}_{6} \mathrm{D}_{5} \mathrm{H}$ is noted as *. Residual $n$ pentane is denoted by ${ }^{\wedge}$.

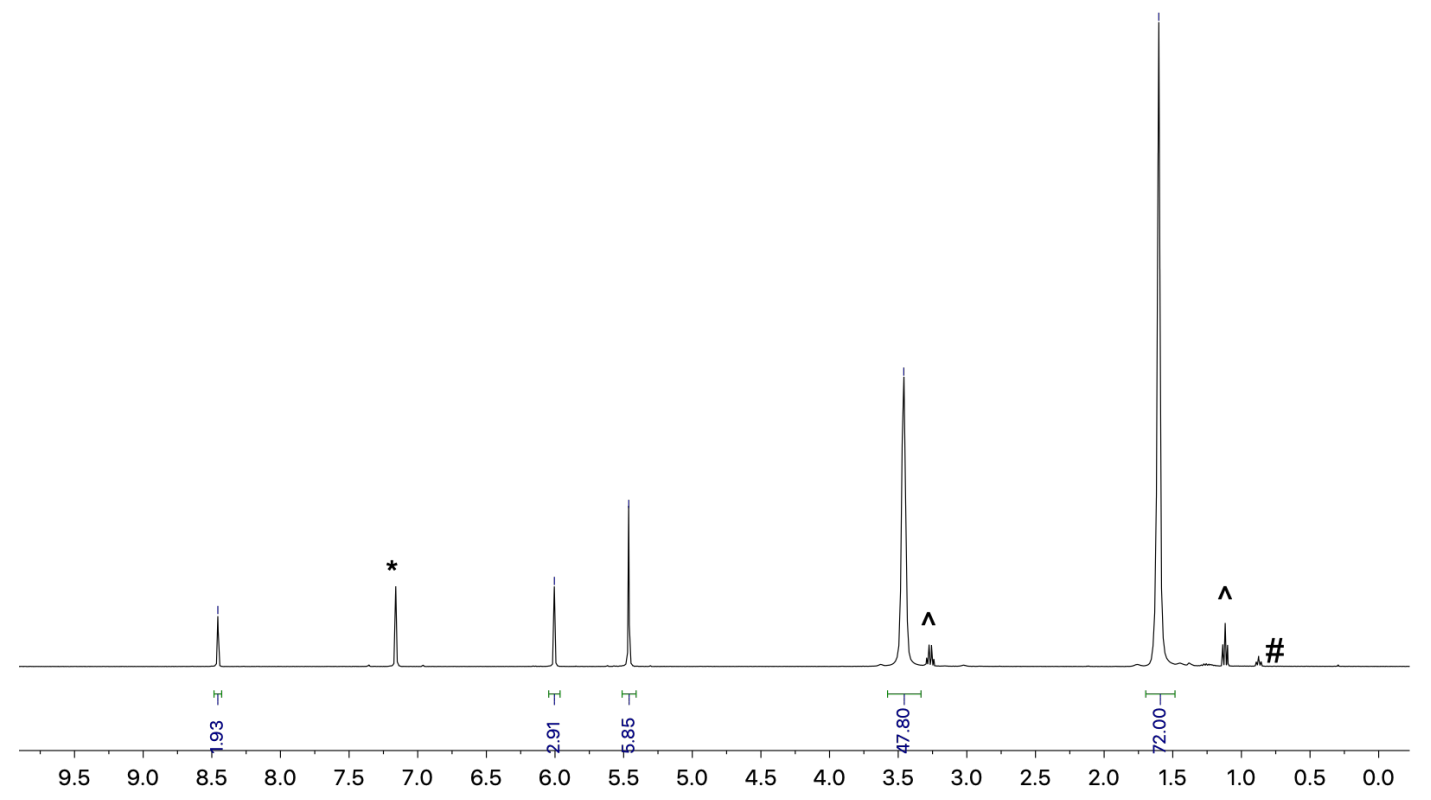

Figure S4. ${ }^{1} \mathrm{H}$ NMR of 2-U(PN)NMes in $\mathrm{C}_{6} \mathrm{D}_{6}$. Peak of $\mathrm{C}_{6} \mathrm{D}_{5} \mathrm{H}$ is noted as *. Residual diethyl ether is denoted by ${ }^{\wedge}$. Residual $n$-pentane is denoted by \#. 


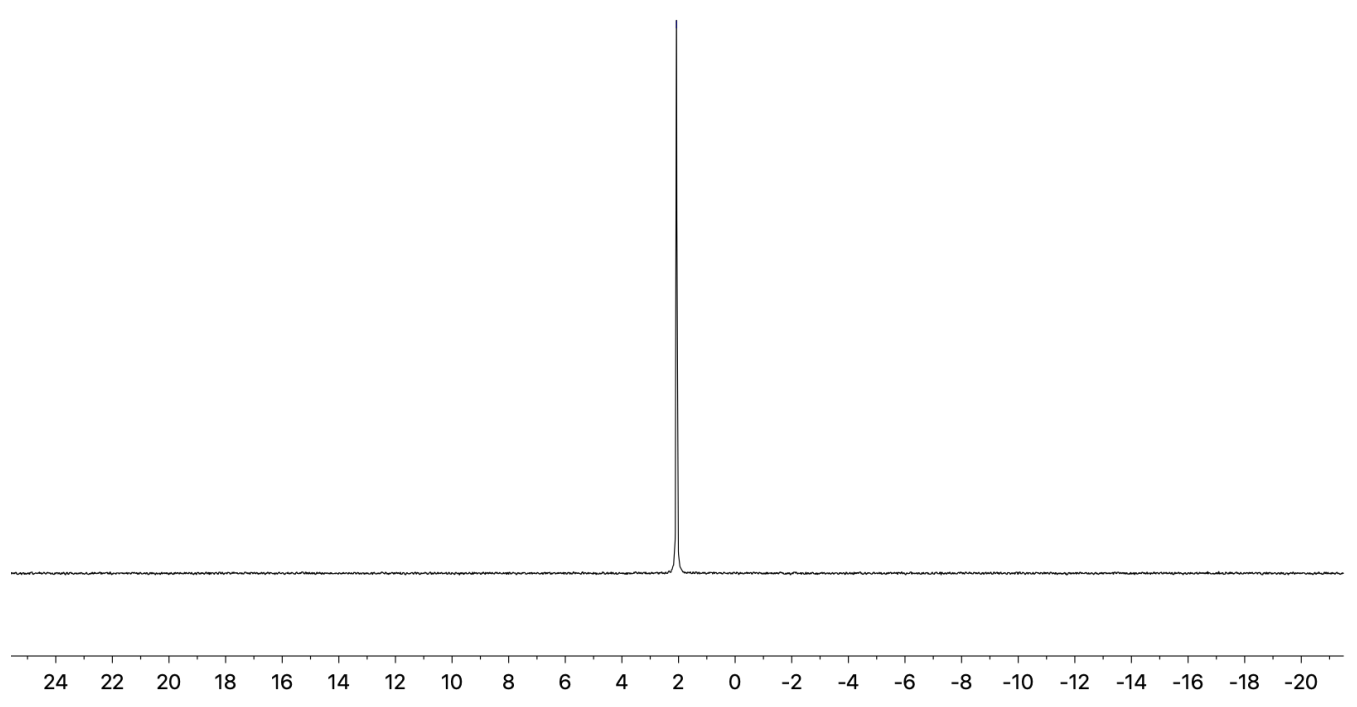

Figure S5. ${ }^{31} \mathrm{P}\left\{{ }^{1} \mathrm{H}\right\}$ NMR of 2-U(PN)NMes in $\mathrm{C}_{6} \mathrm{D}_{6}$.

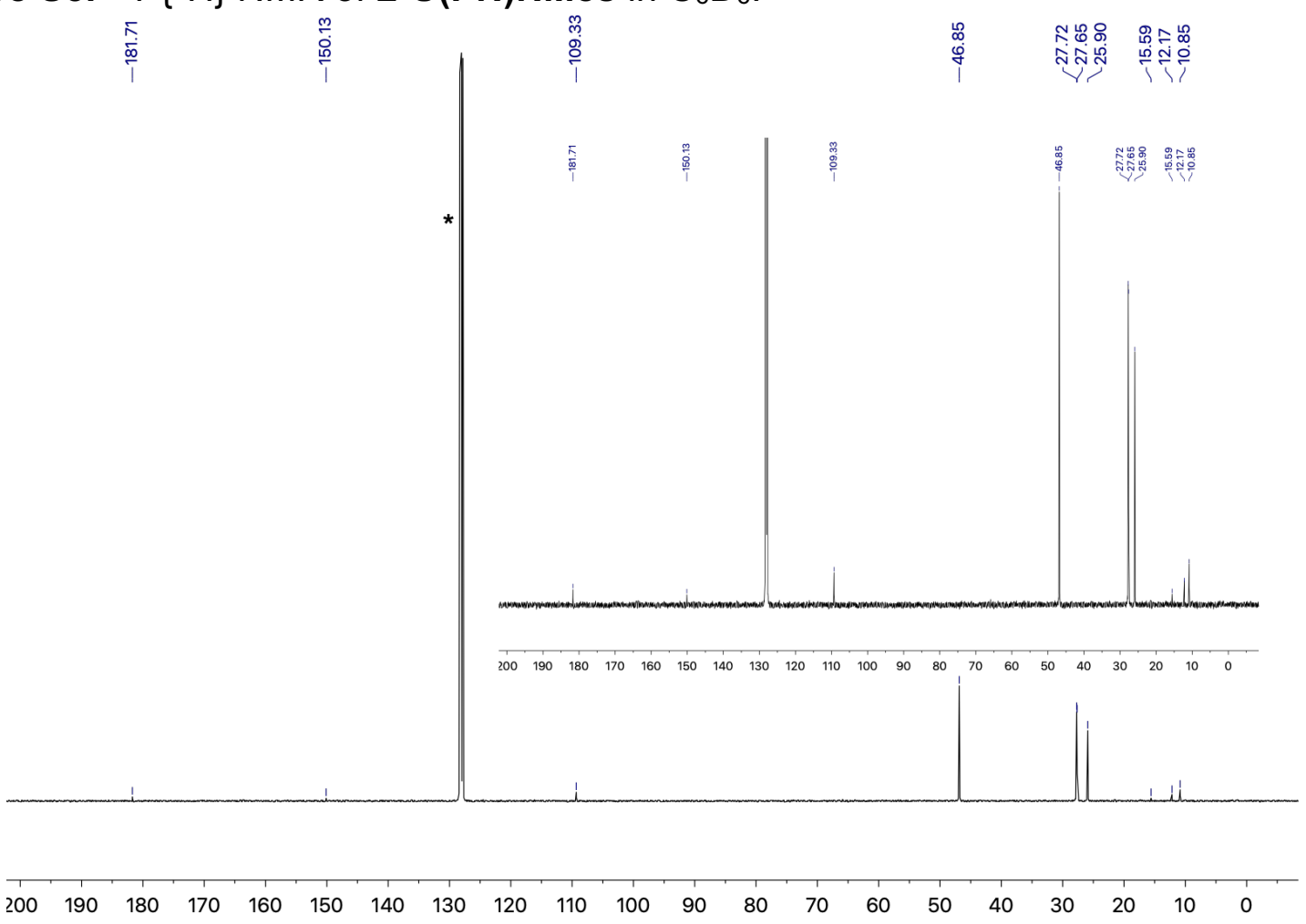

Figure S6. ${ }^{13} \mathrm{C}\left\{{ }^{1} \mathrm{H}\right\}$ NMR of 2-U(PN)NMes in $\mathrm{C}_{6} \mathrm{D}_{6}$. Peak of $\mathrm{C}_{6} \mathrm{D}_{5} \mathrm{H}$ is noted as *. 


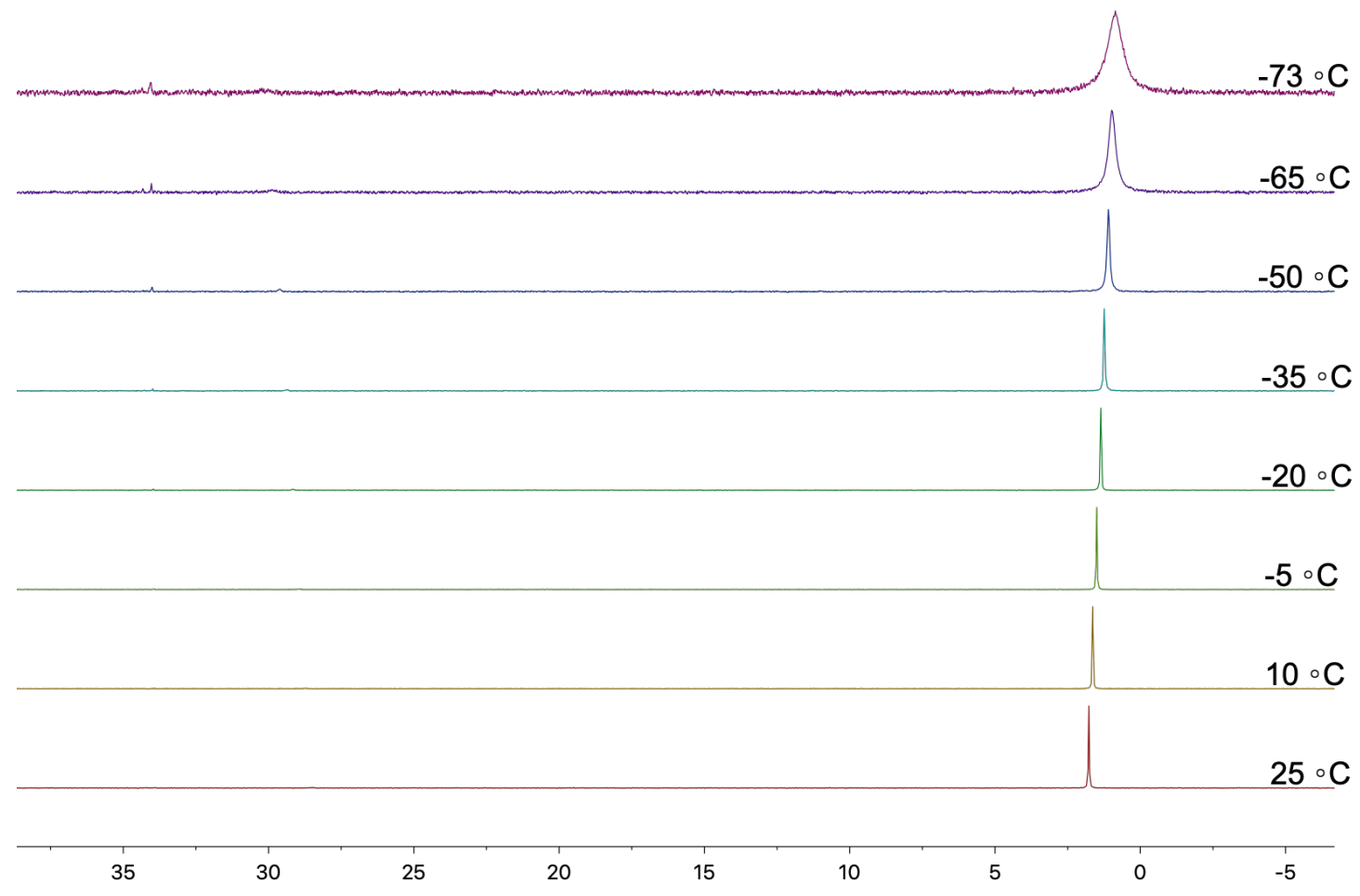

Figure S7. VT- ${ }^{31} \mathrm{P}$ NMR of $2-\mathrm{U}(\mathrm{PN}) \mathrm{NMes}$ in $\mathrm{d}_{8}-\mathrm{THF}$ from $25^{\circ} \mathrm{C}$ to $-73^{\circ} \mathrm{C}$.

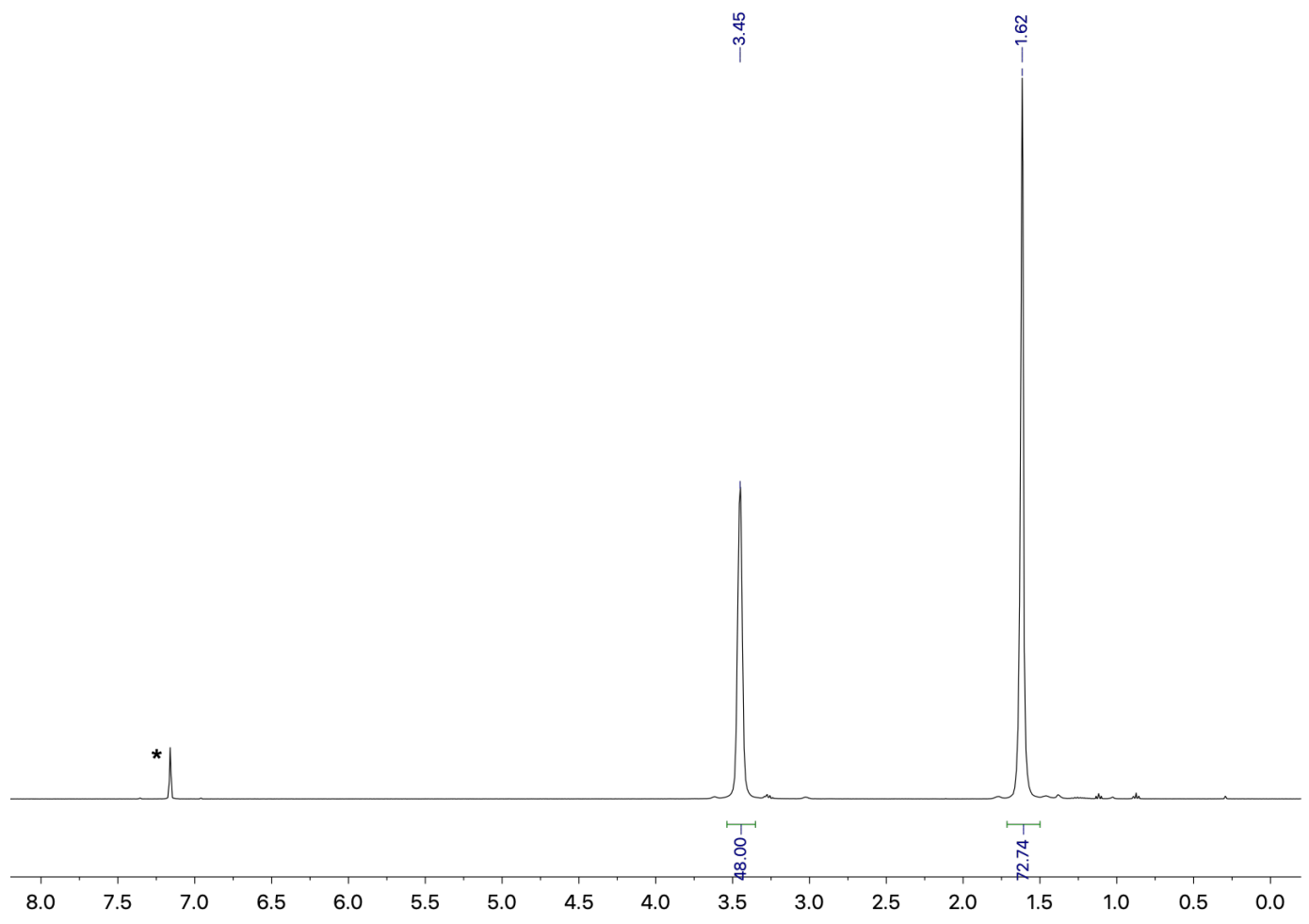

Figure S8. ${ }^{1} \mathrm{H}$ NMR of 2-U(PN)O in $\mathrm{C}_{6} \mathrm{D}_{6}$. Peak of $\mathrm{C}_{6} \mathrm{D}_{5} \mathrm{H}$ is noted as *. 


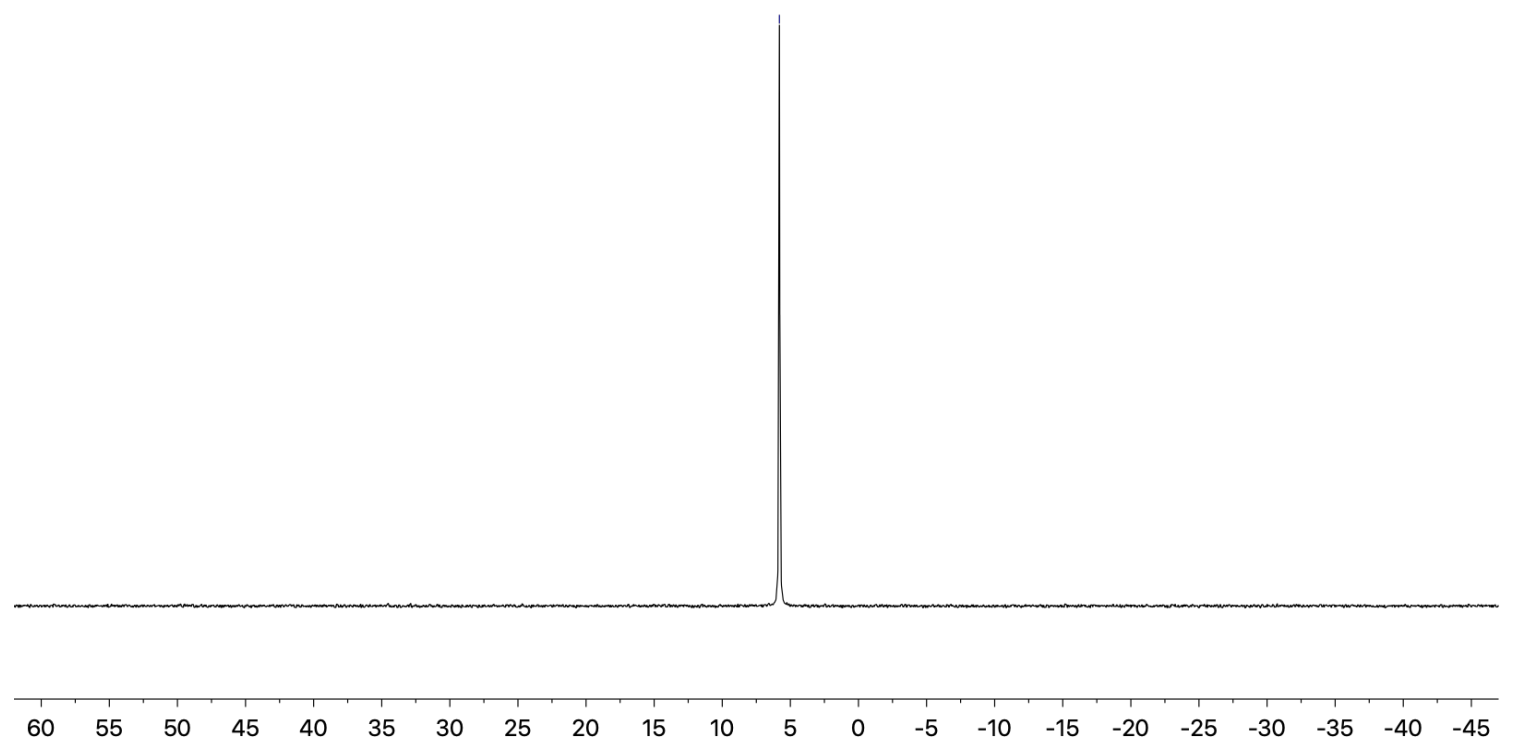

Figure S9. ${ }^{31} \mathrm{P}\left\{{ }^{1} \mathrm{H}\right\}$ NMR of $2-\mathrm{U}(\mathrm{PN}) \mathrm{O}$ in $\mathrm{C}_{6} \mathrm{D}_{6}$.
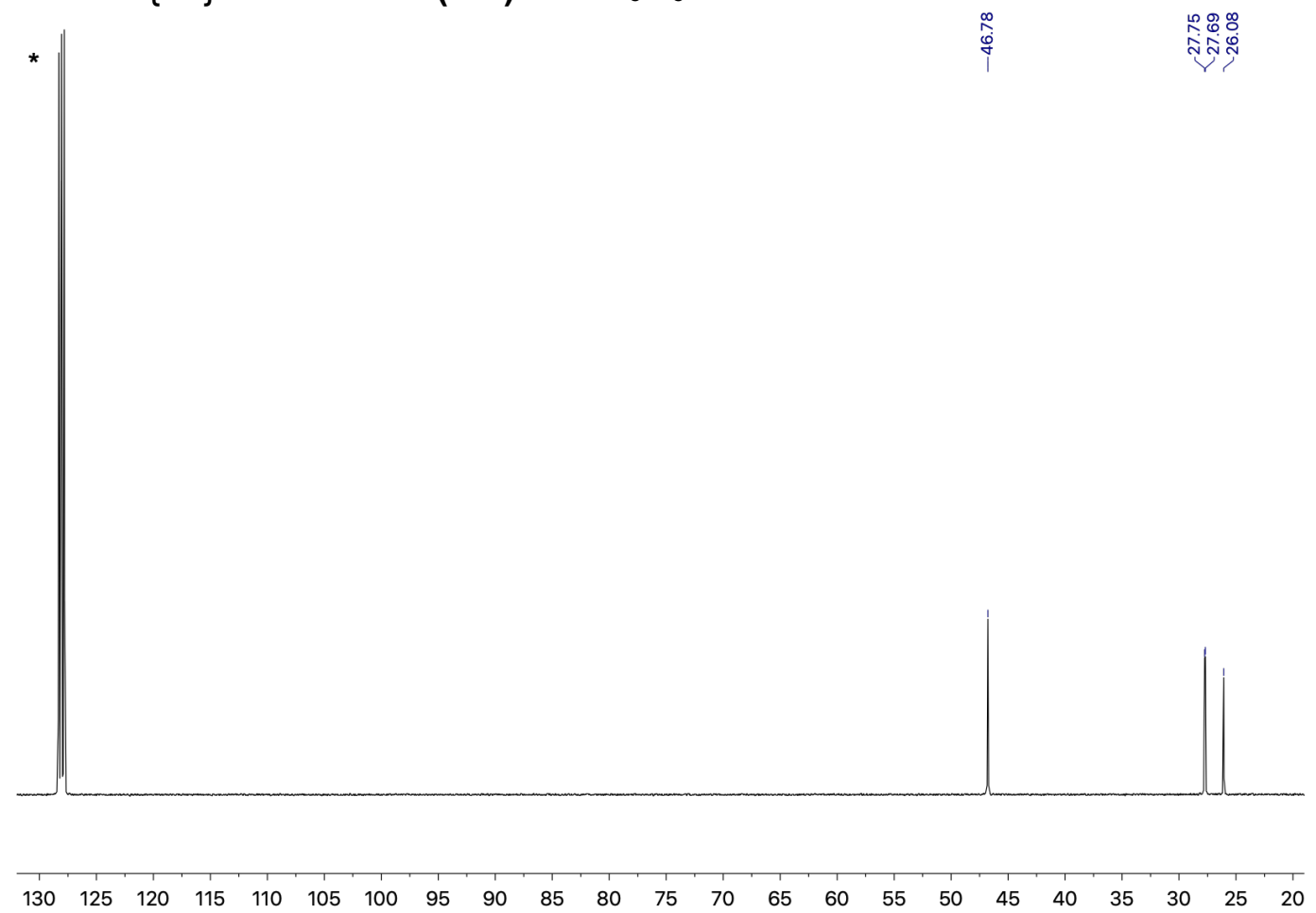

Figure S10. ${ }^{13} \mathrm{C}\left\{{ }^{1} \mathrm{H}\right\}$ NMR of $2-\mathrm{U}(\mathrm{PN}) \mathrm{O}$ in $\mathrm{C}_{6} \mathrm{D}_{6}$. Peak of $\mathrm{C}_{6} \mathrm{D}_{5} \mathrm{H}$ is noted as *. 


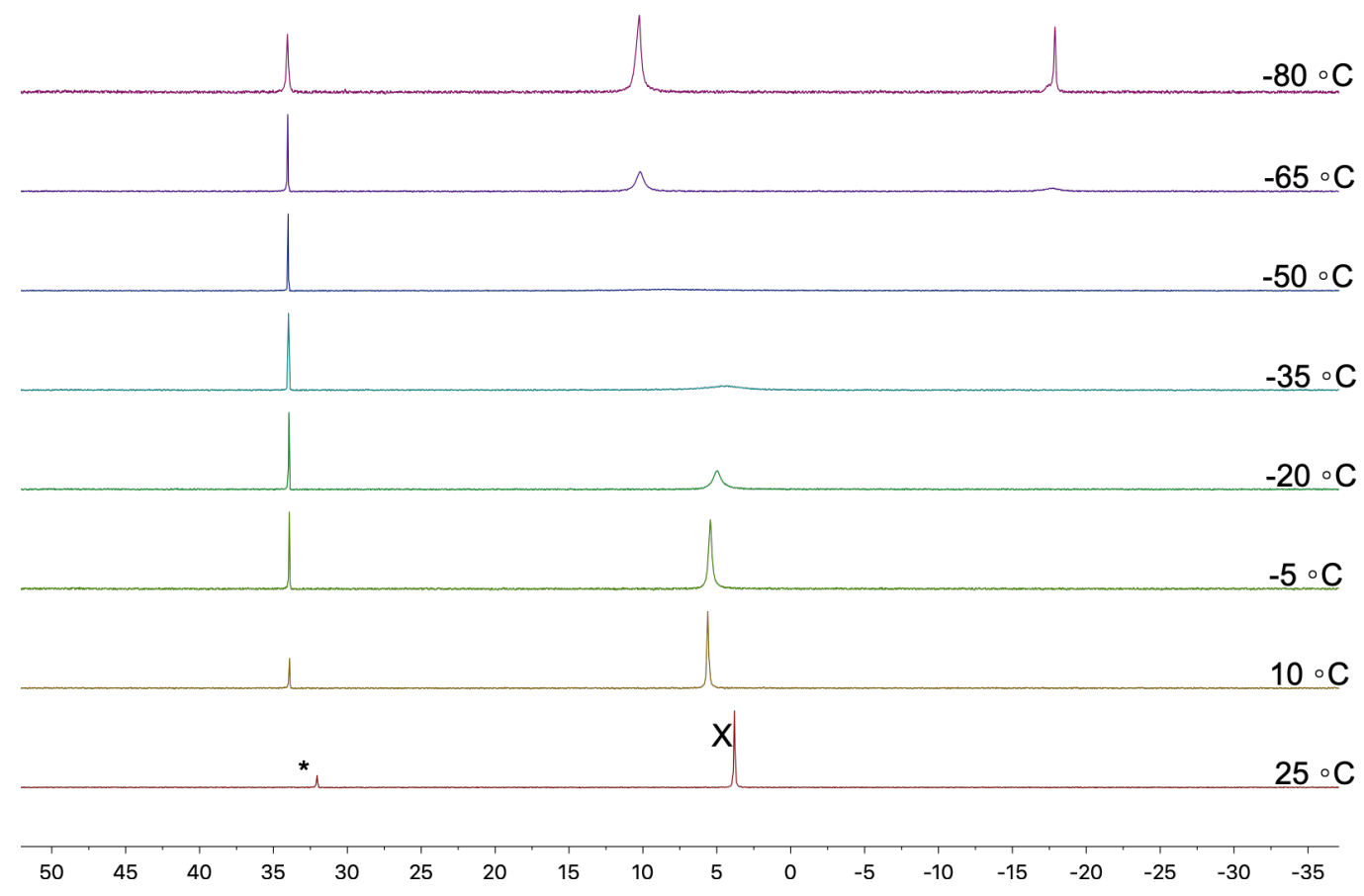

Figure S11. VT- ${ }^{31} \mathrm{P}\left\{{ }^{1} \mathrm{H}\right\}$ NMR of $2-\mathrm{U}(\mathrm{PN}) \mathrm{O}$ in $\mathrm{d}_{8}-\mathrm{THF}$ from $25^{\circ} \mathrm{C}$ to $-80{ }^{\circ} \mathrm{C}$. The symbol, ${ }^{*}$, denotes adventitious protonated ligand, $\left[\mathrm{HNP}(\text { pip })_{3}\right]$, and $\mathrm{X}$ denotes the signal for 2$\mathrm{U}(\mathrm{PN}) \mathrm{O}$ at $25^{\circ} \mathrm{C}$ which splits into two signals around $-65^{\circ} \mathrm{C}$.

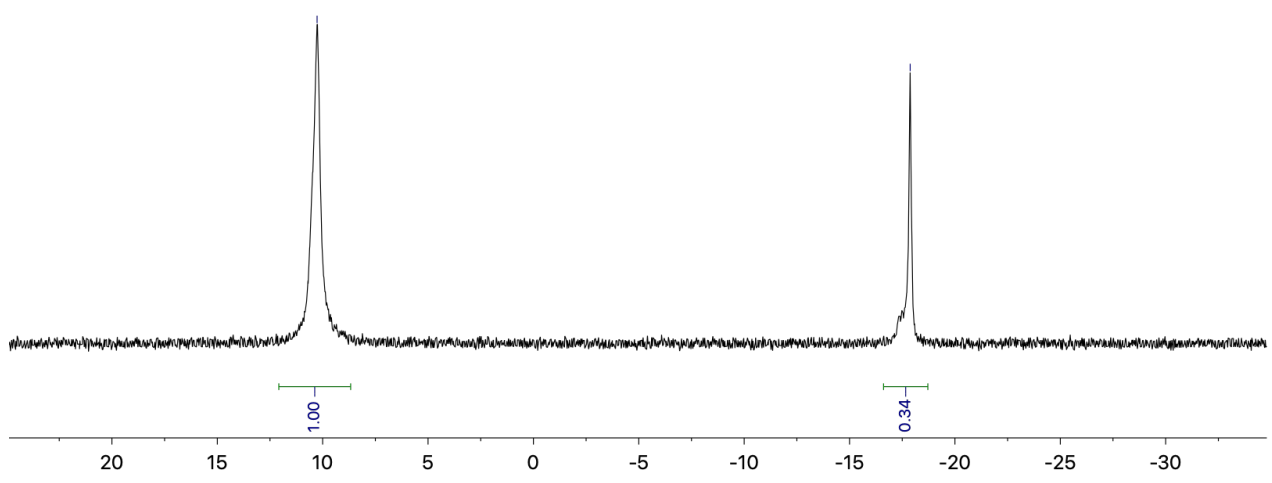

Figure S12. Zoom in of ${ }^{31} \mathrm{P}\{1 \mathrm{H}\} \mathrm{NMR}$ of $2-\mathrm{U}(\mathrm{PN}) \mathrm{O}$ in $\mathrm{d}_{8}-\mathrm{THF}$ at $-80{ }^{\circ} \mathrm{C}$ showing the integration of peaks to be 1:3 denoting a trigonal bipyramidal arrangement of imidophosphorane ligands. 


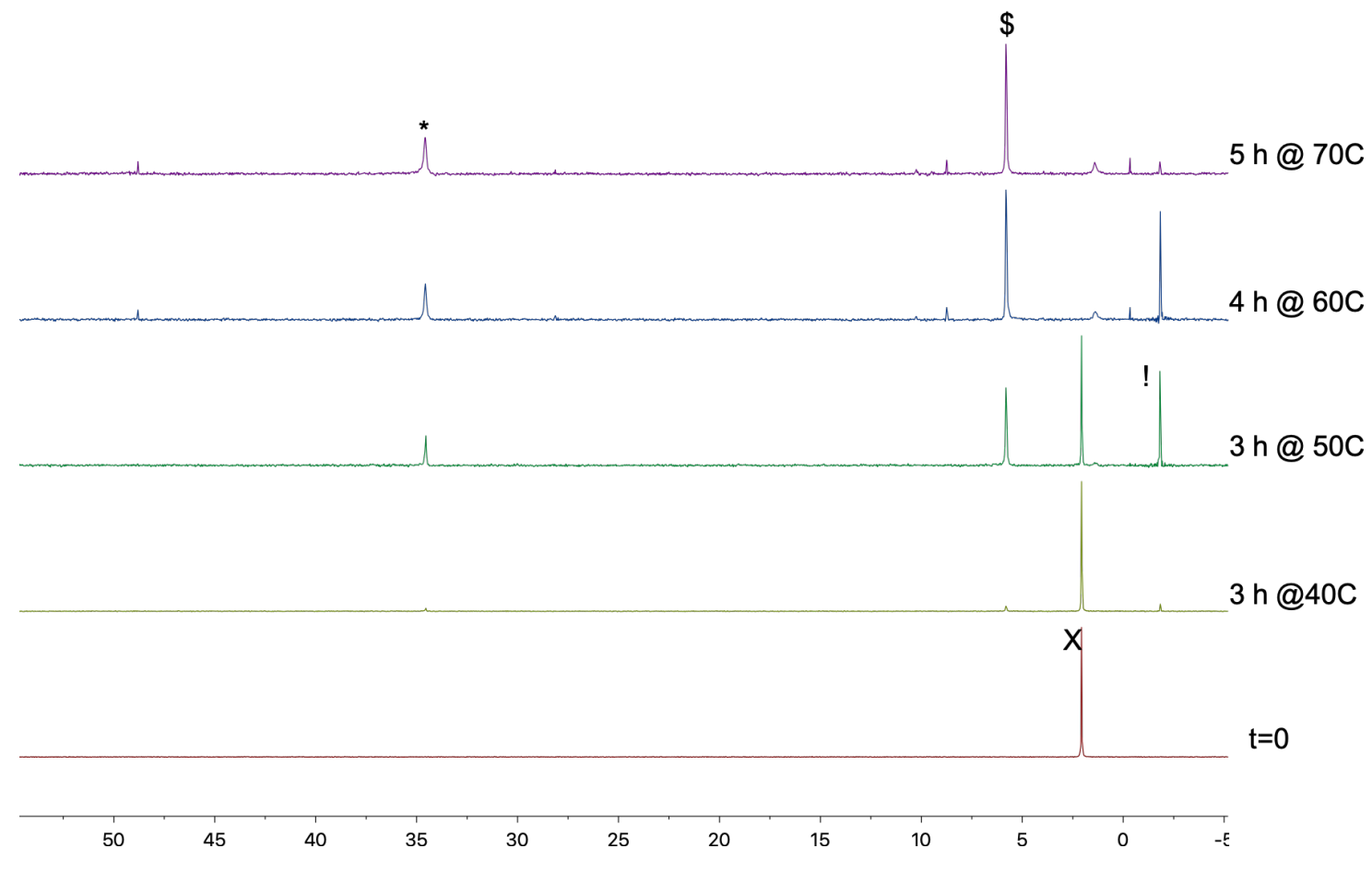

Figure S13. ${ }^{31} \mathrm{P}\left\{{ }^{1} \mathrm{H}\right\}$ NMR of heated reaction of 2-U(PN)NMes with benzophenone in $\mathrm{C}_{6} \mathrm{D}_{6}$ where $\mathrm{X}$ is starting material, $\$$ is the product $\mathbf{2}-\mathrm{U}(\mathbf{P N}) \mathbf{O}$, ! is the proposed intermediate, and * is protonated ligand.

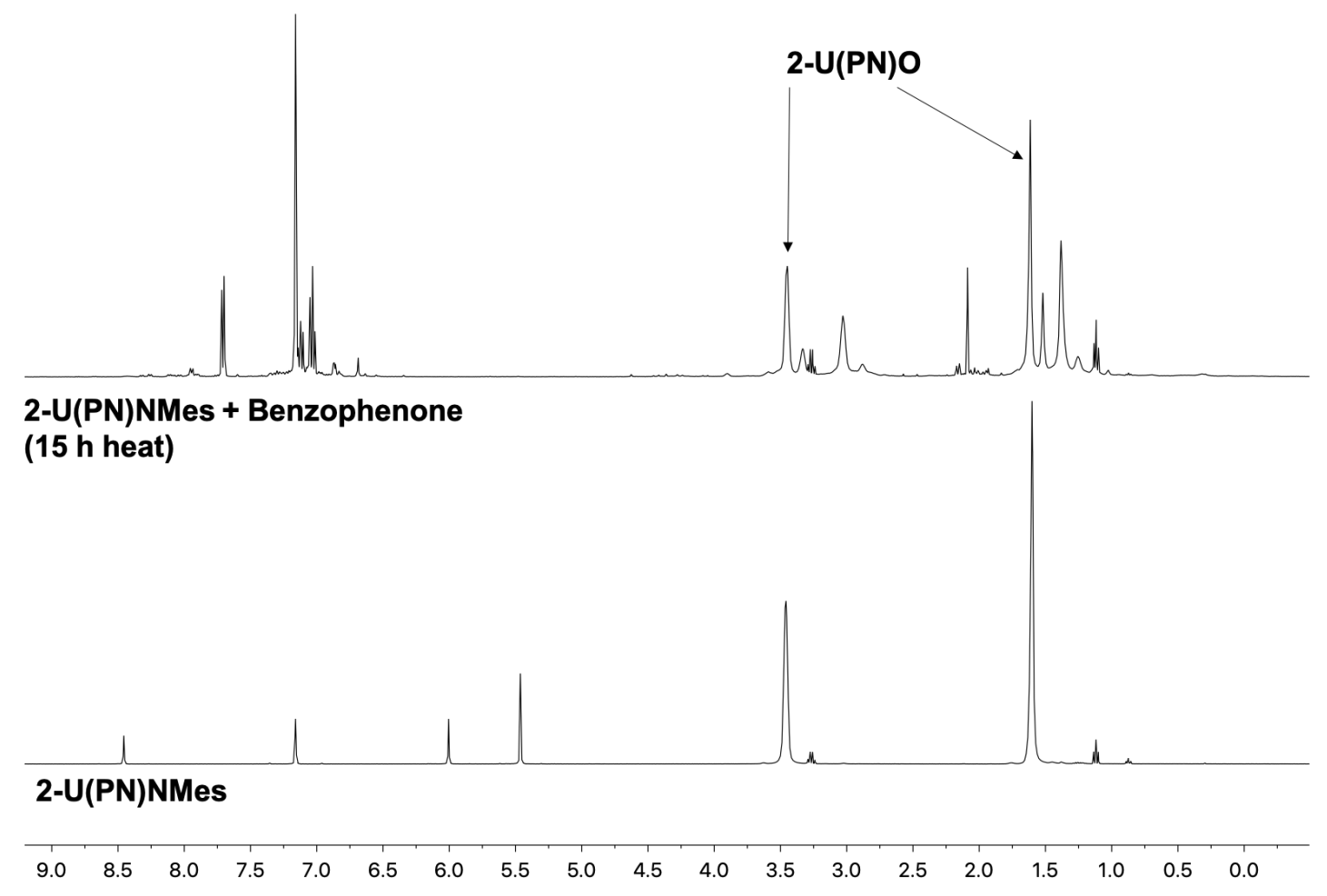

Figure S14. ${ }^{1} \mathrm{H}$ NMR of heated reaction of 2-U(PN)NMes with benzophenone in $\mathrm{C}_{6} \mathrm{D}_{6}$ overlaid with $2-\mathrm{U}(\mathrm{PN}) \mathbf{N M e s}$. Production of $2-\mathrm{U}(\mathrm{PN}) \mathrm{O}$ is marked. 


\section{Crystallographic Analyses}

Crystals suitable for X-ray diffraction were covered in paratone oil in a glove box and transferred to the diffractometer in a $20 \mathrm{~mL}$ capped vial. Crystals were mounted on a loop with paratone oil on a Bruker D8 VENTURE diffractometer. The crystals were cooled and kept at $T=100(2) \mathrm{K}$ during data collections (except for $3-$ following description - which was collected at $T=180(2) \mathrm{K}$ ). The structures were solved with the ShelXT structure solution program using the Intrinsic Phasing solution method and by using Olex2 as the graphical interface. ${ }^{5,6}$ The model was refined with version 2014/7 of XL using Least Squares minimization. ${ }^{7}$

The space group of the crystals of complex $1-\mathbf{U}(\mathbf{P N})$ is $R 3$ with a commensurate modulated supercell. Although the positions and thermal parameters of the $U$ atoms are in good agreement with the diffraction data, the lighter atomic positions were initially in poorer agreement. Disorder along the 3-fold axes smeared the electron densities of the light atoms but a reasonably ordered structure was obtained by refinement of the data as a chiral twin (with large displacement ellipsoids for the lighter atoms). The complex 1U(PN) exhibits whole molecule disorder and twinning. This disorder gave 4-fold superlattice reflections but the supercell did not yield a superior structural model for the complex. It is important to rationalize the strong disorder (which is absent in the other 4coordinate $U$ complexes). The disorder reflects the considerable flexibility of the imidophosphorane ligand but this is not necessarily the source of the disorder. The structure is polar, with one distinct $\mathrm{U}-\mathrm{N}$ bond and three inequivalent $\mathrm{U}-\mathrm{N}$ bonds. The disorder and the four-fold supercell is likely to be due to a small distortion of each of the UL4 groups along the polar axis. This may be due to electronic effects and/or crystal packing. This is probably the reason for the very large displacement parameters of all atoms and this structure is isostructural with the tetrahomoleptic $\mathrm{Ce}^{4+}$ complex, $\left[\mathrm{Ce}\left(\mathrm{NP}(\mathrm{pip})_{3}\right)_{4}\right]$, reported previously.

The cell dimensions and diffraction symmetry of complex 1-U(PN) established the crystal symmetry as trigonal and the Laue symmetry as -3 , and the space group as R3, (No. 146) or R-3 (No. 148). The molecular structure refined best as a chiral structure (with enantiomeric twinning) in the space group R3. The diffraction data was very weak with few reflections and the symmetry operators yield a refinable structural model because of the imposed C3 symmetry on the molecular structure. The space group P1 was tested. The structure will not solve in P1, and the molecular structure could not be freely refined in $\mathrm{P} 1$ because of the absence of imposed crystallographic symmetry. Restraints and constraints would duplicate the symmetry.

The structure, 2-U(PN)O, was solved and the space group $R 3$ (\# 146). All non-hydrogen atoms were refined anisotropically. Hydrogen atom positions were calculated geometrically and refined using the riding model. 2-U(PN)O was refined as a 4component inversion twin. The structure was very disordered. Applying the twin law partially resolved the disorder but the structure refines with very large thermal parameter. We should conclude that the averaged crystal structure does not integrate well into the local structure which must have a much lower local symmetry. The twin law is a 2 -fold 
axis along the direction [110]. The effect of the twin is that it re-orients that molecular structure with the polar axis (along the $\mathrm{U}=\mathrm{O}$ bond) pointing up, so that it will be pointing down. The twin law flips the molecule so that the $U=O$ bond is pointing down (and the $U$ $\mathrm{N}$ pointing up). The twin only partially resolves the disorder, and the molecular structure fails the Hirshfeld test and with low bond precision on C-C bonds

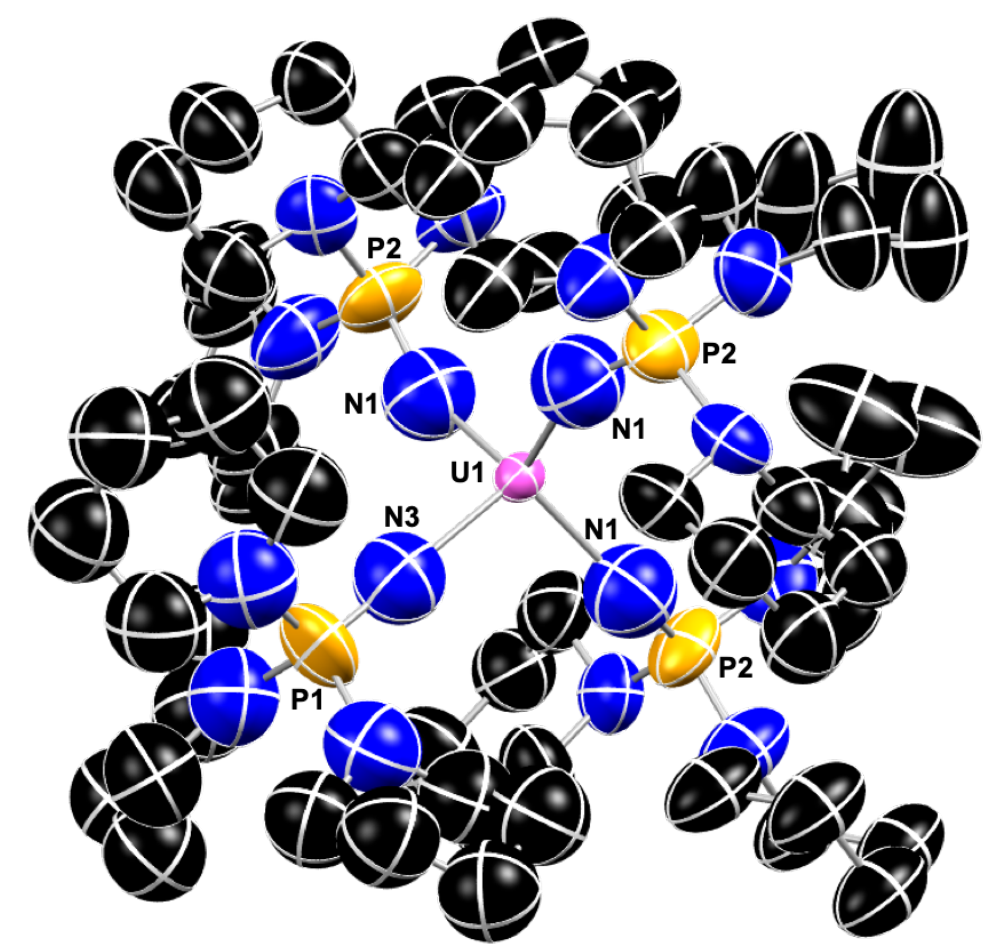

Figure S15. Molecular structure of $1-U(P N)$ with thermal ellipsoids shown at $50 \%$ probability. $\mathrm{H}$ atoms are omitted for clarity. 


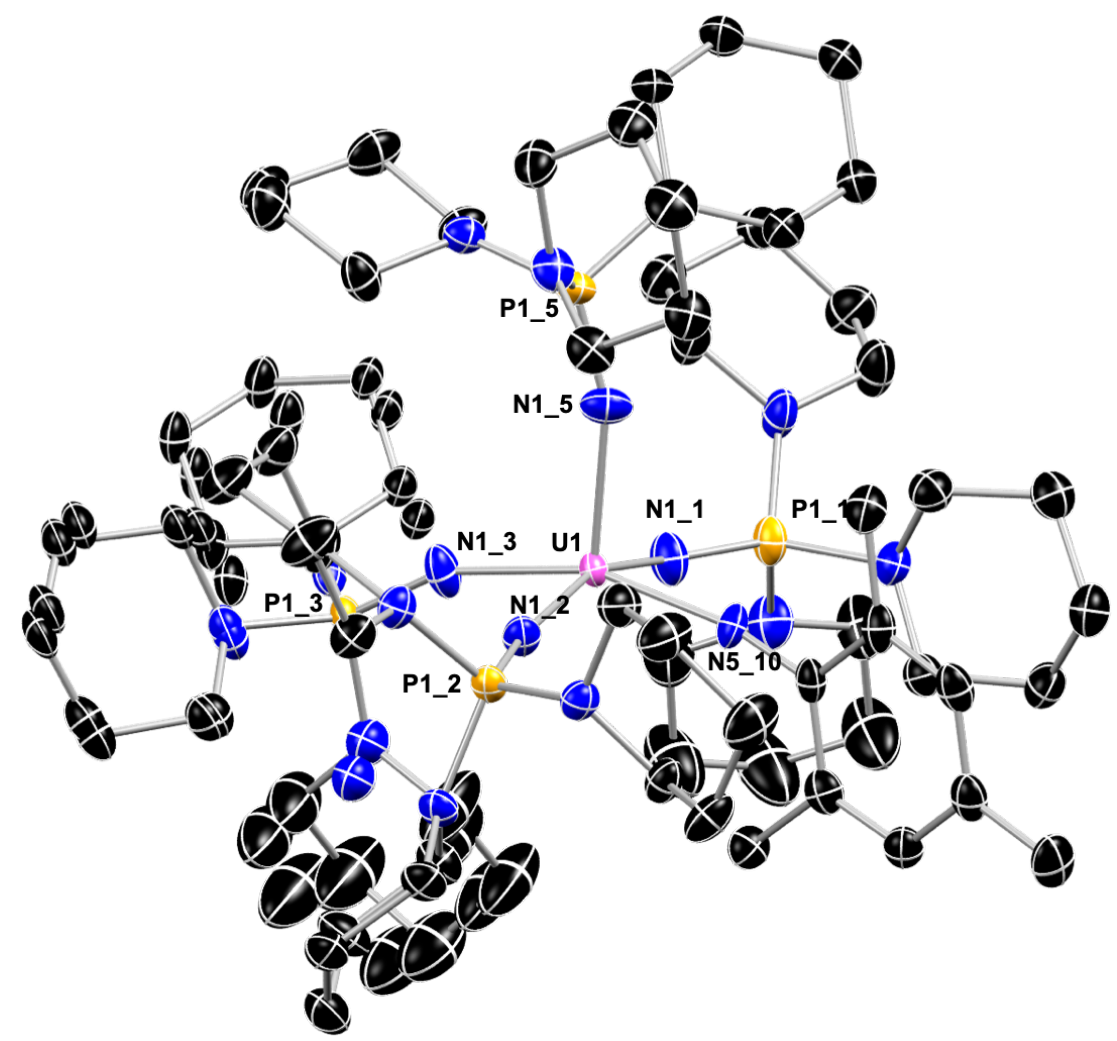

Figure S16. Molecular structure of 2-U(PN)NMes with thermal ellipsoids shown at $50 \%$ probability. $\mathrm{H}$ atoms are omitted for clarity.

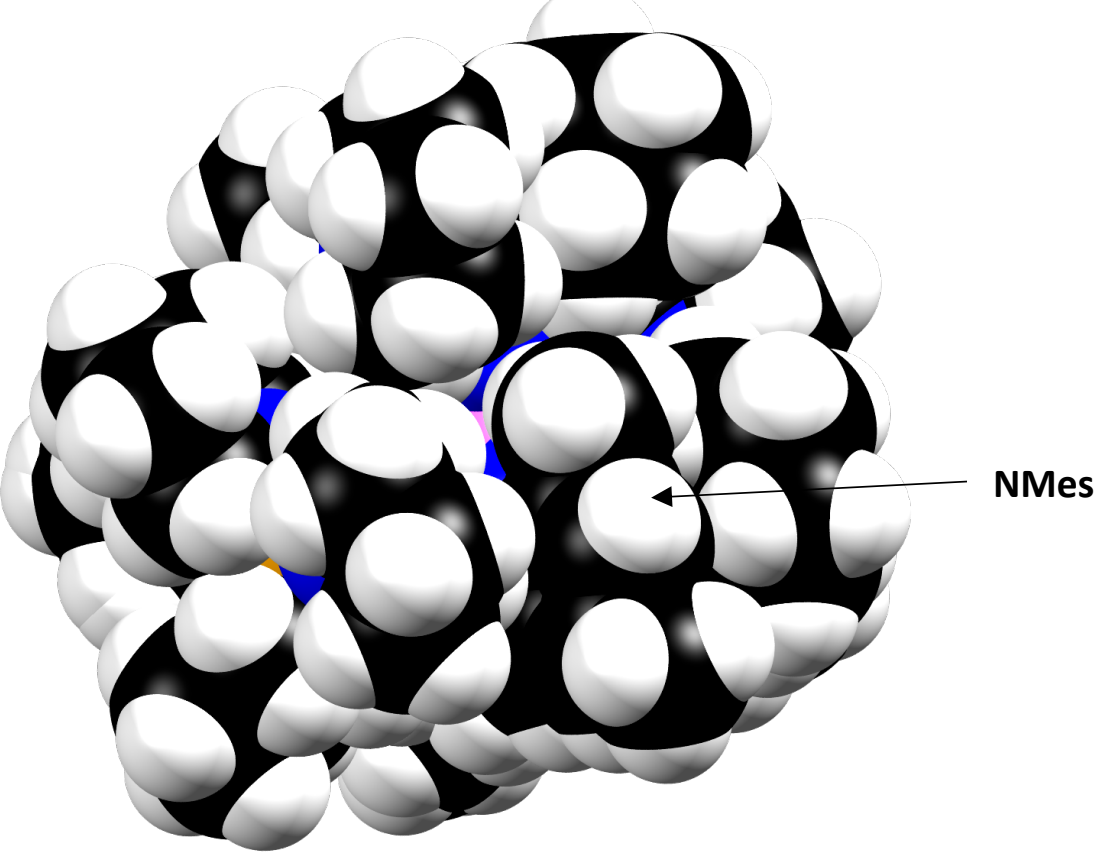

Figure S17. Molecular structure of 2-U(PN)NMes in the same orientation as Figure S16 in space filling model. 


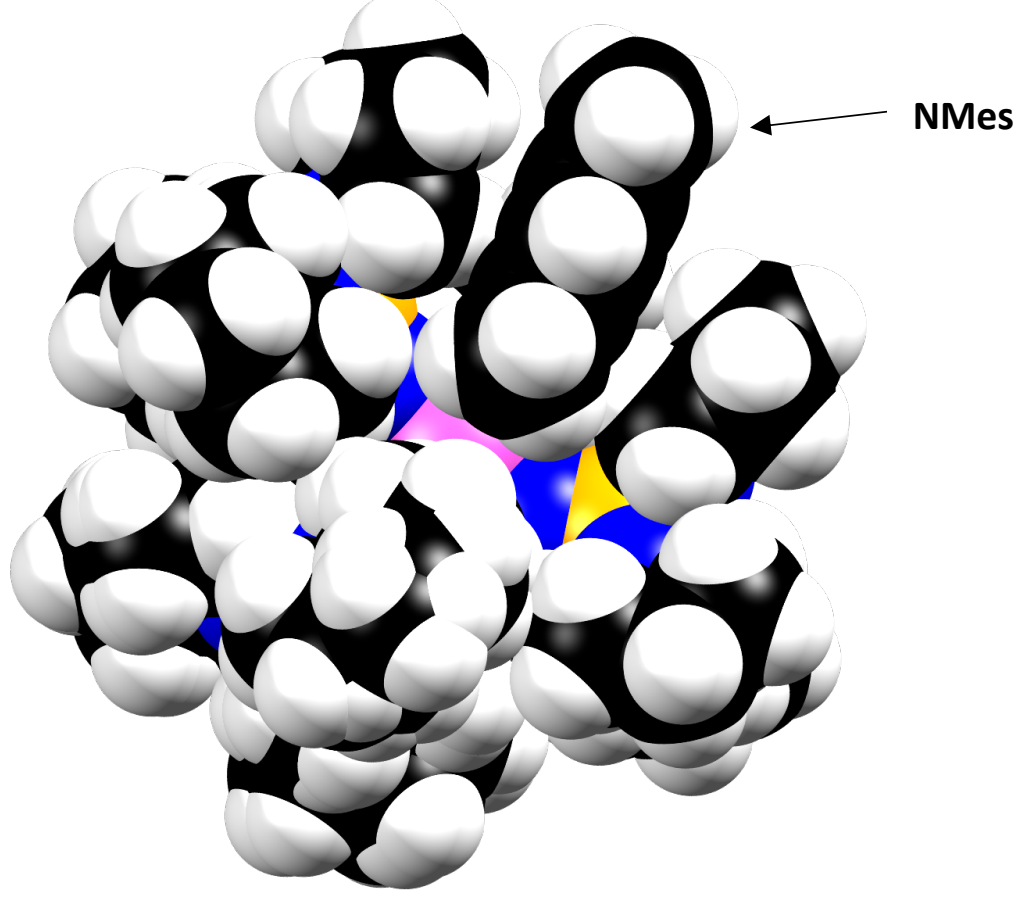

Figure S18. Molecular structure of 2-U(PN)NMes looking up from the base of the pyramid in space filling model.

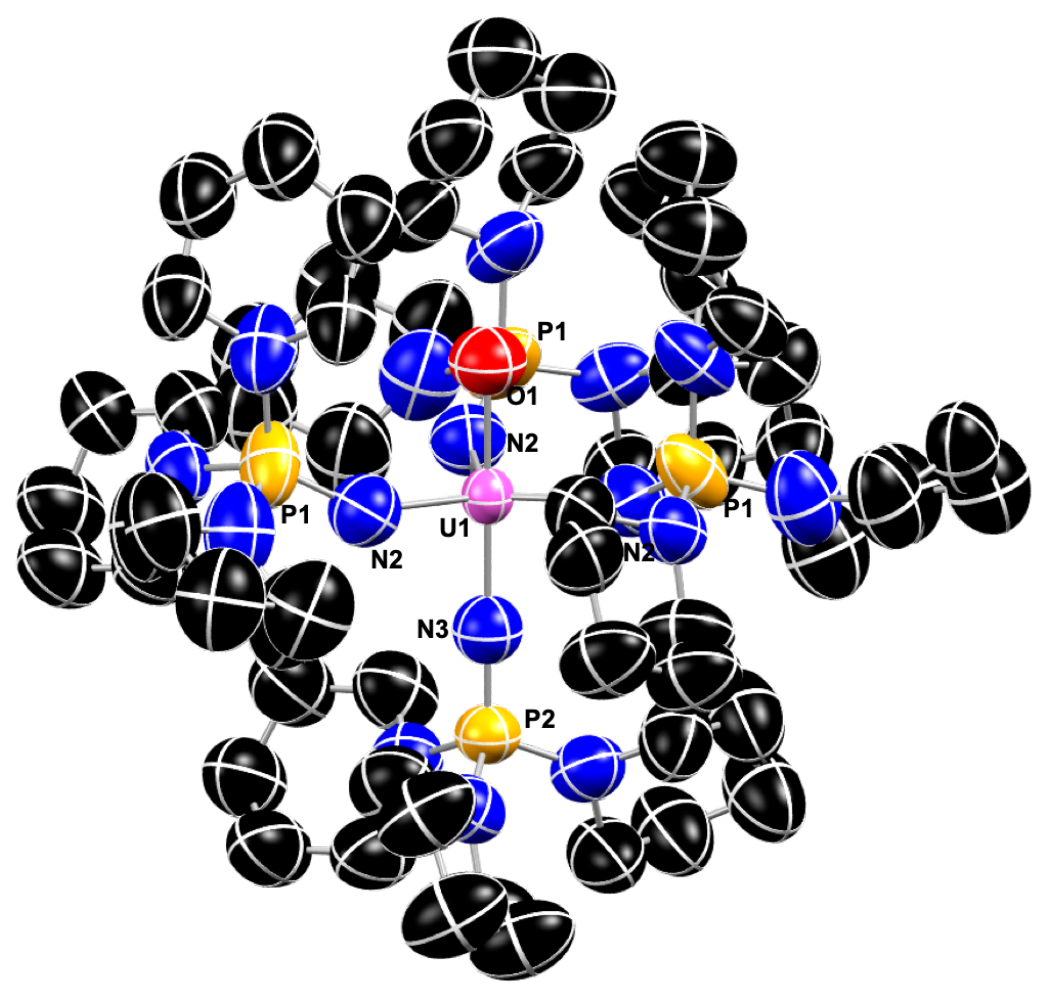

Figure S19. Molecular structure of 2-U(PN)O with thermal ellipsoids shown at $50 \%$ probability. $\mathrm{H}$ atoms are omitted for clarity. 


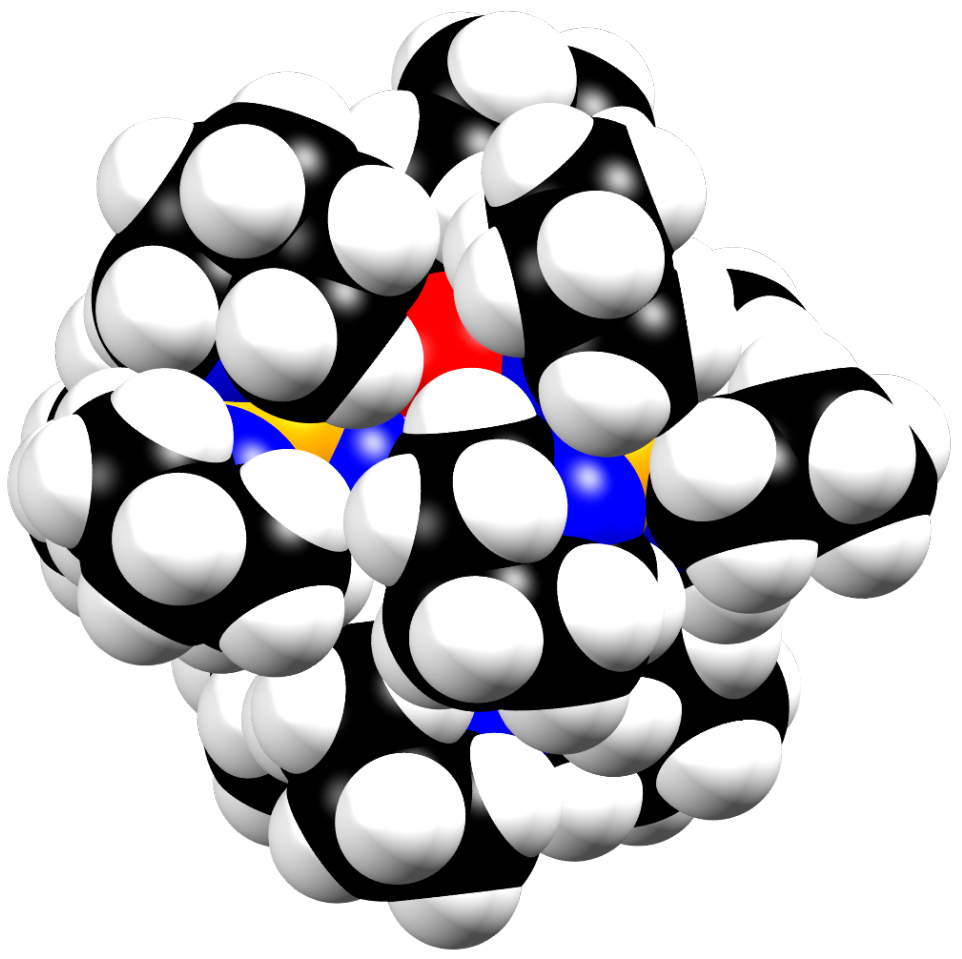

Figure S20. Molecular structure of 2-U(PN)O in the same orientation as Figure $\mathbf{S} 17$ in space filling model.

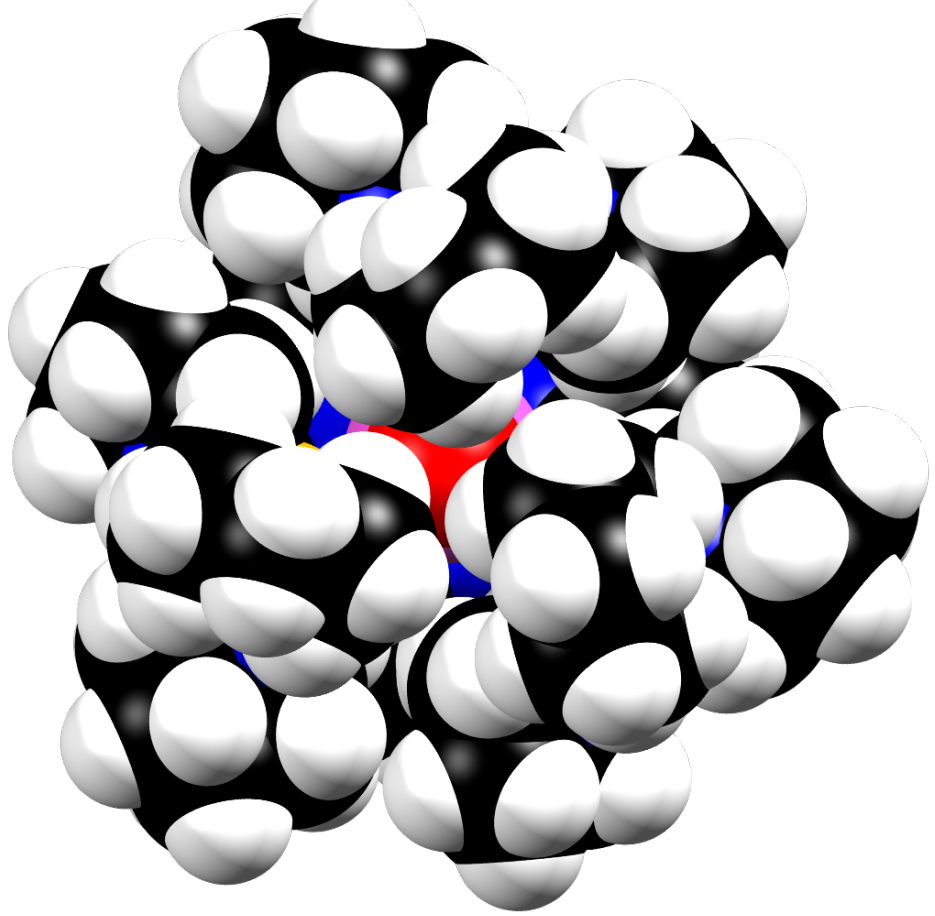

Figure S21. Molecular structure of $2-\mathrm{U}(\mathrm{PN}) \mathrm{O}$ looking down the $\mathrm{C} 3$ axis at $\mathrm{O}$ atom. 
Table S1. Crystallographic Data

\begin{tabular}{|c|c|c|c|}
\hline & $1-U(P N)$ & 2-U(PN)NMes & $2-U(P N) O$ \\
\hline $\begin{array}{l}\text { Empirical Formula } \\
\text { Formula Weiaht }\end{array}$ & $\begin{array}{c}\mathrm{C}_{60} \mathrm{H}_{120} \mathrm{~N}_{16} \mathrm{P}_{4} \mathrm{U} \\
142762\end{array}$ & $\begin{array}{c}\mathrm{C}_{69} \mathrm{H}_{131} \mathrm{UN}_{17} \mathrm{P}_{4} \\
156081\end{array}$ & $\begin{array}{c}\mathrm{C}_{60} \mathrm{H}_{120} \mathrm{~N}_{! 6} \mathrm{P}_{4} \cup \mathrm{U} \\
144362\end{array}$ \\
\hline Temperature (K) & $100(2)$ & $100(2)$ & $103.58(5)$ \\
\hline Crystal System & trigonal & monoclinic & trigonal \\
\hline Space Group & R3 & $\mathrm{P} 22_{1} / \mathrm{c}$ & R3 \\
\hline$a / A$ & $20.6977(9)$ & $14.9753(17)$ & $20.770(3)$ \\
\hline$b / A$ & 20.6977(9) & $22.266(3)$ & $20.770(3)$ \\
\hline$c / \AA$ & 14.1093(7) & $47.714(6)$ & $13.975(2)$ \\
\hline alo & 90 & 90 & 90 \\
\hline$\beta / 0$ & 90 & $97.018(5)$ & 90 \\
\hline r/o & 120 & 90 & 120 \\
\hline Volume/ $\AA^{3}$ & $5234.6(5)$ & 15791(3) & $5220.9(16)$ \\
\hline Z & 3 & 8 & 3 \\
\hline$Z^{\prime}$ & 0.3333 & 2 & 0.3333 \\
\hline$\rho\left(\mathrm{g} / \mathrm{cm}^{3}\right)$ & 1.359 & 1.313 & 1.377 \\
\hline$\mu\left(m m^{-1}\right)$ & 2.465 & 2.186 & 2.474 \\
\hline$F(000)$ & 2232 & 6528 & 2256 \\
\hline Crystal Size $/ \mathrm{mm}^{3}$ & $0.35 \times 0.23 \times 0.17$ & $0.312 \times 0.206 \times 0.192$ & $\begin{array}{c}0.333 \times 0.276 \mathrm{x} \\
0.262\end{array}$ \\
\hline Radiation & $\operatorname{MoK} \alpha(\lambda=0.71073) \mathrm{s}$ & $\operatorname{MoK} \alpha(\lambda=0.71073)$ & $\operatorname{MoK} \alpha(\lambda=0.71073)$ \\
\hline $\begin{array}{l}2 \theta \text { range for data } \\
\text { collection(o) }\end{array}$ & 3.103 to 26.723 & 4.386 to 54.968 & 2.693 to 30.480 \\
\hline Index Ranges & $\begin{array}{c}*-28 \leq h \leq 28,-28 \leq k \leq \\
28,-19 \leq I \leq 19\end{array}$ & $\begin{array}{c}*-16 \leq h \leq 19,-28 \leq k \\
\leq 28,-61 \leq I \leq 61\end{array}$ & $\begin{array}{l}*-26 \leq h \leq 28,-29 \leq 18 \\
k \leq 29,-17 \leq I \leq 18\end{array}$ \\
\hline Reflections Collected & 16181 & 158678 & 27505 \\
\hline Independent Reflections & $\begin{array}{c}4925\left[R_{\text {int }}=0.0328\right. \\
R_{\text {sigma }}=0.0373\end{array}$ & $\begin{array}{c}36167\left[R_{\text {int }}=0.0657\right. \\
R_{\text {sigma }}=0.0503\end{array}$ & $\begin{array}{c}6437\left[R_{\text {int }}=0.0476\right. \\
R_{\text {sigma }}=0.0356\end{array}$ \\
\hline Data/Restraints/Parameters & $16181 / 297 / 245$ & $36167 / 3198 / 1706$ & $6437 / 413 / 251$ \\
\hline Goodness-of-Fit on $F^{2}$ & 1.063 & 1.035 & 1.101 \\
\hline Final $R$ Indexes [I>=2 $\sigma(I)]$ & $\begin{aligned} \mathrm{R}_{1}= & 0.0580, \mathrm{wR} \mathrm{R}_{2}= \\
& 0.1485\end{aligned}$ & $\begin{aligned} \mathrm{R}_{1} & =0.0534 \\
\mathrm{wR}_{2} & =0.1220\end{aligned}$ & $\begin{aligned} \mathrm{R}_{1} & =0.0605 \\
\mathrm{wR} & =0.1498\end{aligned}$ \\
\hline Final R Indexes [all data] & $\begin{aligned} \mathrm{R}_{1}= & 0.0589, \mathrm{wR}_{2}= \\
& 0.1502\end{aligned}$ & $\begin{aligned} \mathrm{R}_{1} & =0.0693 \\
w R_{2} & =0.1312\end{aligned}$ & $\begin{aligned} \mathrm{R}_{1} & =0.0651 \\
w R_{2} & =0.1561\end{aligned}$ \\
\hline $\begin{array}{c}\text { Largest Diff. Peak/Hole/ (e } \\
\left.A^{3}\right)\end{array}$ & $1.829 /-1.375$ & $3.05 /-1.27$ & $1.364 /-0.887$ \\
\hline Completeness to $2 \theta$ & 99.5 & 99.5 & 99.8 \\
\hline
\end{tabular}

Table S2: Bond Lengths in $\AA$ for 1-U(PN).

\begin{tabular}{lll}
\hline Atom & Atom & Length/A \\
\hline U1 & N1 & $2.13(2)$ \\
U1 & N11 & $2.13(2)$ \\
U1 & N12 & $2.13(2)$ \\
U1 & N3 & $2.24(6)$ \\
N1 & P2 & $1.54(2)$ \\
N3 & P1 & $1.49(6)$ \\
P1 & N2_31 & $1.677(15)$ \\
P1 & N2_3 & $1.677(15)$ \\
P1 & N2_32 & $1.677(15)$ \\
P2 & N2_4 & $1.640(13)$ \\
P2 & N2_2 & $1.625(16)$ \\
P2 & N2_1 & $1.689(17)$ \\
N2_4 & C1_4 & $1.464(6)$ \\
N2_4 & C5_4 & $1.464(6)$
\end{tabular}




\begin{tabular}{lll}
\hline Atom & Atom & Length/A \\
\hline C1_4 & C2_4 & $1.526(5)$ \\
C2_4 & C3_4 & $1.526(5)$ \\
C3_4 & C4_4 & $1.524(5)$ \\
C4_4 & C5_4 & $1.523(5)$ \\
N2_2 & C1_2 & $1.464(6)$ \\
N2_2 & C5_2 & $1.464(6)$ \\
C1_2 & C2_2 & $1.525(5)$ \\
C2_2 & C3_2 & $1.526(5)$ \\
C3_2 & C4_2 & $1.524(5)$ \\
C4_2 & C5_2 & $1.525(5)$ \\
N2_1 & C1_1 & $1.464(6)$ \\
N2_1 & C5_1 & $1.464(6)$ \\
C1_1 & C2_1 & $1.524(5)$ \\
C2_1 & C3_1 & $1.525(5)$ \\
C3_1 & C4_1 & $1.525(5)$ \\
C4_1 & C5_1 & $1.524(5)$ \\
N2_3 & C1_3 & $1.464(6)$ \\
N2_3 & C5_3 & $1.464(6)$ \\
C1_3 & C2_3 & $1.523(7)$ \\
C2_3 & C3_3 & $1.527(7)$ \\
C3_3 & C4_3 & $1.525(7)$ \\
C4_3 & C5_3 & $1.524(7)$ \\
1-y,+x-y, +z; ${ }^{2}+y-x,-x,+z$ &
\end{tabular}

Table S3: Bond Angles in ${ }^{\circ}$ for $1-U(P N)$.

\begin{tabular}{llll}
\hline Atom & Atom & Atom & \multicolumn{1}{c}{ Angle $^{\circ}$} \\
\hline N1 & U1 & N11 & $114.7(4)$ \\
N1 & U1 & N12 & $114.7(4)$ \\
N11 & U1 & N12 & $114.7(4)$ \\
N1 & U1 & N3 & $103.5(6)$ \\
N11 & U1 & N3 & $103.5(6)$ \\
N12 & U1 & N3 & $103.5(6)$ \\
P2 & N1 & U1 & $152.5(14)$ \\
P1 & N3 & U1 & 180.0 \\
N3 & P1 & N2_3 & $112.6(6)$ \\
N3 & P1 & N2_32 & $112.6(6)$ \\
N3 & P1 & N2_31 & $112.6(6)$ \\
N2_3 & P1 & N2_3 & $106.2(6)$ \\
N2_31 & P1 & N2_32 & $106.2(6)$ \\
N2_31 & P1 & N2_3 & $106.2(6)$ \\
N1 & P2 & N2_4 & $107.0(10)$ \\
N1 & P2 & N2_2 & $115.2(10)$ \\
N1 & P2 & N2_1 & $119.0(10)$ \\
N2_4 & P2 & N2_1 & $106.8(8)$ \\
N2_2 & P2 & N2_4 & $107.1(8)$ \\
N2_2 & P2 & N2_1 & $100.9(7)$ \\
C1_4 & N2_4 & P2 & $121.8(9)$ \\
C5_4 & N2_4 & P2 & $122.4(10)$ \\
C5_4 & N2_4 & C1_4 & $111.9(11)$ \\
N2_4 & C1_4 & C2_4 & $105.2(11)$ \\
C1_4 & C2_4 & C3_4 & $109.7(8)$ \\
& & &
\end{tabular}




\begin{tabular}{|c|c|c|c|}
\hline Atom & Atom & Atom & Angle ${ }^{\circ}$ \\
\hline$\overline{\text { C4_44 }}$ & C3_4 & C2_4 & $104.6(12)$ \\
\hline C5_4 & C4_4 & C3_4 & $112.2(11)$ \\
\hline N2_4 & C5_4 & C4_4 & 104.1(10) \\
\hline $\mathrm{C} 1^{-} 2$ & $\mathrm{~N} 2-2$ & $\mathrm{P}^{-}$ & 112.6(10) \\
\hline C1_2 & N2_2 & C5_2 & 111.4(10) \\
\hline C5_2 & N2_2 & $\mathrm{P} 2$ & $129.3(10)$ \\
\hline N2_2 & C1_2 & C2_2 & 105.5(10) \\
\hline $\mathrm{C}^{-}{ }^{-} 2$ & $\mathrm{C} 2-2$ & $\mathrm{C} 3{ }^{-} 2$ & $110.0(10)$ \\
\hline C4_2 & C3_2 & $\mathrm{C} 2 \_2$ & $104.8(12)$ \\
\hline C3_2 & C4_2 & C5_2 & $111.8(11)$ \\
\hline N2_2 & C5_2 & C4_2 & $103.5(10)$ \\
\hline $\mathrm{C}^{-1} 1$ & $\mathrm{~N} 2-1$ & $\mathrm{P}^{-}$ & 108.8(10) \\
\hline $\mathrm{C} 1-1$ & N2_-1 & C5_1 & 110.8(10) \\
\hline C5_1 & N2_1 & P2 & 114.1(11) \\
\hline $\mathrm{N} 2{ }^{-} 1$ & $\mathrm{C}^{-1} 1$ & C2 1 & 105.3(11) \\
\hline $\mathrm{C} 1{ }^{-} 1$ & $\mathrm{C} 2-1$ & $\mathrm{C} 3{ }^{-} 1$ & $110.2(10)$ \\
\hline C4_1 & C3_1 & C2_1 & $104.8(12)$ \\
\hline C5_1 & C4_1 & C3_1 & $111.8(11)$ \\
\hline N2_1 & C5_1 & C4_1 & 103.8(10) \\
\hline C1_3 & N2_3 & P1 & $118.7(11)$ \\
\hline C5_3 & N2_3 & P1 & $124.9(12)$ \\
\hline C5_3 & N2_3 & C1_3 & $111.3(11)$ \\
\hline $\mathrm{N} 23$ & $\mathrm{C}^{-} 3$ & $\mathrm{C} 22^{-} 3$ & 105.4(11) \\
\hline $\mathrm{C}_{1}^{-} 3$ & $\mathrm{C} 2{ }^{-} 3$ & $\mathrm{C} 3{ }^{-} 3$ & $110.4(9)$ \\
\hline C4_3 & C3_3 & C2_3 & $104.3(13)$ \\
\hline C5_3 & C4_3 & C3_3 & 111.7(9) \\
\hline N2_3 & C5_3 & C4_3 & $104.2(11)$ \\
\hline \multicolumn{4}{|c|}{${ }^{1}-\mathrm{y},+\mathrm{x}-\mathrm{y},+\mathrm{z} ;{ }^{2}+\overline{\mathrm{y}}-\mathrm{x},-\mathrm{x},+\mathrm{z}$} \\
\hline
\end{tabular}

Table S4: Bond Lengths in $\AA$ for 2-U(PN)NMes.

\begin{tabular}{llllll}
\hline Atom & Atom & Length/A & Atom & Atom & Length/A \\
\hline U1 & N5_10 & $1.991(4)$ & C11_4 & C12_4 & $1.495(3)$ \\
U1 & N1_1 & $2.146(2)$ & C12_4 & C13_4 & $1.524(3)$ \\
U1 & N1_2 & $2.1492(18)$ & C13_4 & C14_4 & $1.528(3)$ \\
U1 & N1_3 & $2.148(4)$ & C14_4 & C15_4 & $1.491(3)$ \\
U1 & N1_4 & $2.136(4)$ & P1_5 & N1_5 & $1.5505(17)$ \\
U1 & N1_5 & $2.1254(19)$ & P1_5 & N2_5 & $1.6798(16)$ \\
U2 & N5_11 & $1.995(4)$ & P1_5 & N3_5 & $1.6729(17)$ \\
U2 & N1_6 & $2.138(2)$ & P1_5 & N4_5 & $1.6682(17)$ \\
U2 & N1_7 & $2.1485(18)$ & N2_5 & C1_5 & $1.473(2)$ \\
U2 & N1_8 & $2.1441(18)$ & N2_5 & C5_5 & $1.473(2)$ \\
U2 & N1_9 & $2.1312(19)$ & N3_5 & C6_5 & $1.469(2)$ \\
N5_10 & C16_10 & $1.370(6)$ & N3_5 & C10_5 & $1.463(2)$ \\
C16_10 & C17_10 & $1.416(7)$ & N4_5 & C11_5 & $1.464(2)$ \\
C16_10 & C21_10 & $1.424(7)$ & N4_5 & C15_5 & $1.465(2)$ \\
C17_10 & C18_10 & $1.379(7)$ & C1_5 & C2_5 & $1.520(3)$ \\
C17_10 & C22_10 & $1.500(7)$ & C2_5 & C3_5 & $1.522(3)$ \\
C18_10 & C19_10 & $1.397(7)$ & C3_5 & C4_5 & $1.526(3)$ \\
C19_10 & C20_10 & $1.397(8)$ & C4_5 & C5_5 & $1.518(3)$ \\
C19_10 & C23_10 & $1.524(7)$ & C6_5 & C7_5 & $1.490(3)$
\end{tabular}




\begin{tabular}{|c|c|c|c|c|c|}
\hline C20_10 & C21_10 & $1.385(7)$ & C7_5 & C8_5 & $1.530(3)$ \\
\hline C21_10 & C24_10 & $1.508(8)$ & C8_5 & C9_5 & $1.519(3)$ \\
\hline N5_11 & C16_11 & $1.367(6)$ & C9_5 & C10_5 & $1.493(3)$ \\
\hline C16_11 & C17_11 & $1.435(7)$ & C11_5 & C12_5 & $1.495(3)$ \\
\hline C16_11 & C21_11 & $1.421(7)$ & C12_5 & C13_5 & $1.523(3)$ \\
\hline C17_11 & C18_11 & $1.395(7)$ & C13_5 & C14_5 & $1.528(3)$ \\
\hline C17_11 & C22_11 & $1.505(7)$ & C14_5 & C15_5 & $1.491(3)$ \\
\hline C18_11 & C19_11 & $1.397(8)$ & P1_6 & N1_6 & $1.5536(17)$ \\
\hline C19_11 & C20_11 & $1.388(8)$ & P1_6 & N2_6 & $1.6768(16)$ \\
\hline C19_11 & C23_11 & $1.515(7)$ & P1_6 & N3_6 & $1.6691(17)$ \\
\hline C20_11 & C21_11 & $1.394(7)$ & P1_6 & N4_6 & $1.6647(17)$ \\
\hline C21_11 & C24_11 & $1.497(7)$ & N2_6 & C1_6 & $1.466(2)$ \\
\hline P1_1 & N1_1 & $1.5501(17)$ & N2_6 & C5_6 & $1.468(2)$ \\
\hline P1_1 & $\mathrm{N} 2{ }_{-} 1$ & $1.6730(16)$ & N3_6 & C6_6 & $1.459(2)$ \\
\hline P1_1 & N3_1 & $1.6675(17)$ & N3_6 & C10_6 & $1.456(2)$ \\
\hline P1_1 & N4_1 & $1.6755(17)$ & N4_6 & C11_6 & $1.455(2)$ \\
\hline N2_1 & C1_1 & $1.464(2)$ & N4_6 & C15_6 & $1.456(2)$ \\
\hline N2_1 & C5_1 & $1.465(2)$ & C1_6 & C2_6 & $1.523(3)$ \\
\hline N3_1 & C6_1 & $1.456(2)$ & C2_6 & C3_6 & $1.521(3)$ \\
\hline N3_1 & C10_1 & $1.456(2)$ & C3_6 & C4_6 & $1.525(3)$ \\
\hline N4_1 & C11_1 & $1.464(2)$ & C4_6 & C5_6 & $1.521(3)$ \\
\hline N4_1 & C15_1 & $1.461(2)$ & C6_6 & C7_6 & $1.492(3)$ \\
\hline C1_1 & C2_1 & $1.523(3)$ & C7_6 & C8_6 & $1.530(3)$ \\
\hline C2_1 & C3_1 & $1.521(3)$ & C8_6 & C9_6 & $1.518(3)$ \\
\hline C3_1 & C4_1 & $1.524(3)$ & C9_6 & C10_6 & $1.493(3)$ \\
\hline C4_1 & C5_1 & $1.522(3)$ & C11_6 & C12_6 & $1.494(3)$ \\
\hline C6_1 & C7_1 & $1.491(3)$ & C12_6 & C13_6 & $1.523(3)$ \\
\hline C7_1 & C8_1 & $1.529(3)$ & C13_6 & C14_6 & $1.528(3)$ \\
\hline C8_1 & C9_1 & $1.518(3)$ & C14_6 & C15_6 & $1.491(3)$ \\
\hline C9_1 & C10_1 & $1.494(3)$ & P1_7 & N1_7 & $1.5493(16)$ \\
\hline C11_1 & C12_1 & $1.494(3)$ & P1_7 & N2_7 & $1.6740(16)$ \\
\hline C12_1 & C13_1 & $1.524(3)$ & P1_7 & N3_7 & $1.6680(17)$ \\
\hline C13_1 & C14_1 & $1.528(3)$ & P1_7 & N4_7 & $1.6780(17)$ \\
\hline C14_1 & C15_1 & $1.489(3)$ & N2_7 & C1_7 & $1.464(2)$ \\
\hline P1_2 & N1_2 & $1.5481(16)$ & N2_7 & C5_7 & $1.472(2)$ \\
\hline P1_2 & N2_2 & $1.6660(16)$ & N3_7 & C6_7 & $1.456(2)$ \\
\hline P1_2 & N3_2 & $1.6748(17)$ & N3_7 & C10_7 & $1.456(2)$ \\
\hline P1_2 & N4_2 & $1.6796(17)$ & N4_7 & C11_7 & $1.468(2)$ \\
\hline N2_2 & C1_2 & $1.460(2)$ & N4_7 & C15_7 & $1.469(2)$ \\
\hline N2_2 & C5_2 & $1.465(2)$ & C1_7 & C2_7 & $1.523(3)$ \\
\hline N3_2 & C6_2 & $1.460(2)$ & C2_7 & C3_7 & $1.521(3)$ \\
\hline N3_2 & C10_2 & $1.462(2)$ & C3_7 & C4_7 & $1.525(3)$ \\
\hline N4_2 & C11_2 & $1.468(2)$ & C4_7 & C5_7 & $1.519(3)$ \\
\hline N4_2 & C15_2 & $1.466(2)$ & C6_7 & C7_7 & $1.492(3)$ \\
\hline C1_2 & C2_z & $1.525(3)$ & C7_7 & C8_7 & $1.530(3)$ \\
\hline C2_2 & C3_2 & $1.520(3)$ & C8_7 & C9_7 & $1.519(3)$ \\
\hline C3_2 & C4_2 & $1.524(3)$ & C9_7 & C10_7 & $1.494(3)$ \\
\hline C4_2 & C5_2 & $1.522(3)$ & C11_7 & C12_7 & $1.492(3)$ \\
\hline C6_2 & C7_2 & $1.492(3)$ & C12_7 & C13_7 & $1.525(3)$ \\
\hline
\end{tabular}




\begin{tabular}{|c|c|c|c|c|c|}
\hline C7_2 & C8_2 & $1.529(3)$ & C13_7 & C14_7 & $1.529(3)$ \\
\hline C8_2 & C9_2 & $1.519(3)$ & C14_7 & C15_7 & $1.487(3)$ \\
\hline C9_2 & C10_2 & $1.495(3)$ & P1_- 8 & N1_ē & $1.5482(17)$ \\
\hline C11_2 & C12_2 & $1.493(3)$ & P1_8 & N2_8 & $1.6692(16)$ \\
\hline C12_2 & C13_2 & $1.524(3)$ & P1_8 & N3_8 & $1.6841(17)$ \\
\hline C13_2 & C14_2 & $1.529(3)$ & P1_8 & N4_8 & $1.6669(17)$ \\
\hline C14_2 & C15_2 & $1.489(3)$ & $\mathrm{N} 2{ }^{-} 8$ & C1_8 & $1.466(2)$ \\
\hline P1_ 3 & N1_3 & $1.5514(17)$ & N2_8 & C5_8 & $1.464(2)$ \\
\hline P1_3 & N2_3 & $1.6784(17)$ & N3_8 & C6_8 & $1.469(2)$ \\
\hline P1_3 & N3_3 & $1.6700(17)$ & N3_8 & C10_8 & $1.468(2)$ \\
\hline P1_3 & N4_3 & $1.6667(17)$ & N4_8 & C11_8 & $1.459(2)$ \\
\hline N2_3 & C1_3 & $1.469(2)$ & N4_8 & C15_8 & $1.454(2)$ \\
\hline $\mathrm{N} 2{ }_{-}^{-} 3$ & C5_3 & $1.468(2)$ & C1_8 & $\mathrm{C} 2{ }_{-} \overline{8}$ & $1.524(3)$ \\
\hline N3_3 & C6_3 & $1.461(2)$ & $\mathrm{C} 2{ }^{-} 8$ & C3_8 & $1.521(3)$ \\
\hline N3_3 & C10_3 & $1.459(2)$ & C3_8 & C4_8 & $1.524(3)$ \\
\hline N4_3 & C11_3 & $1.459(2)$ & C4_8 & C5_8 & $1.522(3)$ \\
\hline N4_3 & C15_3 & $1.457(2)$ & C6_8 & C7_8 & $1.490(3)$ \\
\hline C1_3 & C2_ 3 & $1.522(3)$ & C7_8 & C8_8 & $1.530(3)$ \\
\hline C2_3 & C3_3 & $1.522(3)$ & C8_8 & C9_8 & $1.520(3)$ \\
\hline C3_3 & C4_3 & $1.525(3)$ & C9_8 & C10_8 & $1.492(3)$ \\
\hline C4_3 & C5_3 & $1.520(3)$ & C11̄_8 & C12_8 & $1.496(3)$ \\
\hline C6_3 & C7_3 & $1.491(3)$ & C12_8 & C13_8 & $1.524(3)$ \\
\hline C7_3 & C8_3 & $1.529(3)$ & C13_8 & C14_8 & $1.527(3)$ \\
\hline C8_3 & C9_3 & $1.519(3)$ & C14_8 & C15_8 & $1.491(3)$ \\
\hline C9_3 & C10_3 & $1.493(3)$ & P1_ $\overline{9}$ & N1_ēg & $1.5498(17)$ \\
\hline C11__3 & C12_3 & $1.495(3)$ & P1_9 & N2_9 & $1.6809(16)$ \\
\hline C12 3 & $\mathrm{C} 133$ & $1.523(3)$ & $\mathrm{P} 19$ & N3 9 & $1.6721(17)$ \\
\hline C13_3 & C14_3 & $1.528(3)$ & P1_9 & N4_9 & $1.6668(17)$ \\
\hline C14_3 & C15_3 & $1.491(3)$ & N2_9 & C1_9 & $1.472(2)$ \\
\hline $\mathrm{P} 1 \_\overline{4}$ & N1_ $\overline{4}$ & $1.5526(17)$ & N2_9 & C5_9 & $1.471(2)$ \\
\hline P1_4 & $\mathrm{N} 2{ }_{-}^{-} 4$ & $1.6789(17)$ & N3_9 & C6_9 & $1.469(2)$ \\
\hline P1_4 & N3_4 & $1.6675(17)$ & N3_9 & C10_9 & $1.465(2)$ \\
\hline P1_4 & N4_4 & $1.6661(17)$ & N4_9 & C11_9 & $1.462(2)$ \\
\hline N2_4 & C1_4 & $1.468(2)$ & N4_9 & C15_9 & $1.460(2)$ \\
\hline $\mathrm{N} 2{ }_{-}^{-} 4$ & C5_4 & $1.468(2)$ & C1_9 & C2_ $-\overline{9}$ & $1.521(3)$ \\
\hline N3_4 & C6_4 & $1.458(2)$ & C2_9 & C3_9 & $1.522(3)$ \\
\hline N3_4 & C10_4 & $1.455(2)$ & C3_9 & C4_9 & $1.525(3)$ \\
\hline N4_4 & C11_4 & $1.457(2)$ & C4_9 & C5_9 & $1.519(3)$ \\
\hline N4_4 & C15_4 & $1.456(2)$ & C6_9 & C7_9 & $1.493(3)$ \\
\hline C1_4 & C2 $2-\overline{4}$ & $1.522(3)$ & C7_9 & C8_9 & $1.530(3)$ \\
\hline C2_4 & C3_4 & $1.522(3)$ & C8_9 & C9_9 & $1.518(3)$ \\
\hline C3_4 & C4 44 & $1.525(3)$ & C9_9 & C10_9 & $1.494(3)$ \\
\hline C4_4 & C5_4 & $1.520(3)$ & C11__9 & C12_9 & $1.495(3)$ \\
\hline C6_4 & C7_4 & $1.491(3)$ & C12_9 & C13_9 & $1.524(3)$ \\
\hline C7_4 & C8_4 & $1.530(3)$ & C13_9 & C14_9 & $1.527(3)$ \\
\hline C8_4 & $\mathrm{C}_{-}^{-} 4$ & $1.519(3)$ & C14_9 & C15_9 & $1.490(3)$ \\
\hline C9_4 & C10_4 & $1.493(3)$ & & & \\
\hline
\end{tabular}


Table S5: Bond Angles in ${ }^{\circ}$ for 2-U(PN)NMes.

\begin{tabular}{|c|c|c|c|c|c|c|c|}
\hline Atom & Atom & Atom & Angle $^{\circ}$ & Atom & Atom & Atom & Angle $^{\circ}$ \\
\hline N5_10 & U1 & N1_1 & $87.67(14)$ & N2_4 & C5_4 & C4_4 & $110.61(17)$ \\
\hline N5_10 & U1 & N1_2 & $86.17(14)$ & N3_4 & C6_4 & C7_- 4 & $113.27(18)$ \\
\hline N5_10 & U1 & N1_3 & $157.67(18)$ & C6_4 & C7_4 & C8_4 & $112.1(2)$ \\
\hline N5_10 & U1 & N1_4 & $143.4(2)$ & C9_4 & C8_4 & C7_4 & $110.36(19)$ \\
\hline N5_10 & U1 & N1_5 & $100.32(15)$ & C10_4 & $\mathrm{C}^{-}{ }_{-}^{-} 4$ & C8_4 & $111.8(2)$ \\
\hline N1_1 & U1 & N1_2 & $160.82(7)$ & N3_ 4 & C10_4 & C9_4 & $113.32(18)$ \\
\hline N1_1 & U1 & N1_3 & $86.90(16)$ & N4_4 & C11_4 & $\mathrm{C} 1 \overline{2} \_4$ & $113.28(18)$ \\
\hline N1_3 & U1 & N1_2 & $91.95(14)$ & C11__4 & C12_4 & C13_4 & $111.6(2)$ \\
\hline N1_4 & U1 & N1_1 & $84.73(18)$ & C12_4 & C13_4 & C14_4 & $110.0(2)$ \\
\hline N1_4 & U1 & N1_2 & $89.45(16)$ & C15_4 & C14_4 & C13_4 & $112.0(2)$ \\
\hline N1_5 & U1 & N1_1 & $98.99(11)$ & N4_ $\overline{4}$ & C15_4 & C14_4 & $113.42(19)$ \\
\hline N1_5 & U1 & N1_2 & $99.98(11)$ & N1_5 & P1_ 5 & N2_ 5 & $119.38(10)$ \\
\hline N1_5 & U1 & N1_3 & $101.92(15)$ & N1_5 & P1_5 & N3_5 & $111.73(10)$ \\
\hline N1_5 & U1 & N1_4 & $116.22(18)$ & N1_5 & P1_5 & N4_5 & $112.09(10)$ \\
\hline N5_11 & U2 & N1_6 & $88.81(14)$ & N3_5 & P1_5 & N2_5 & $102.18(8)$ \\
\hline N5_11 & U2 & N1_7 & $145.20(14)$ & N4_5 & P1_5 & N2_5 & $101.57(9)$ \\
\hline N5_11 & U2 & N1_8 & $87.10(14)$ & N4_5 & P1_5 & N3_5 & $108.86(8)$ \\
\hline N5_11 & U2 & N1_9 & $102.21(14)$ & P1_5 & N1_5 & $\mathrm{U} 1^{-}$ & $161.28(16)$ \\
\hline N1_6 & U2 & N1_7 & $87.84(11)$ & C1_5 & N2_5 & P1_5 & 115.96(13) \\
\hline N1_6 & U2 & N1_8 & $167.14(7)$ & C5_5 & N2_5 & P1_5 & $116.09(13)$ \\
\hline N1_8 & U2 & N1_7 & $88.58(9)$ & C5_5 & N2_5 & C1_5 & $110.73(16)$ \\
\hline N1_9 & U2 & N1_6 & $95.90(11)$ & C6_5 & N3_5 & P1_5 & $119.12(13)$ \\
\hline N1_9 & U2 & N1_7 & $112.59(11)$ & C10_5 & N3_5 & P1_5 & $123.27(14)$ \\
\hline N1_9 & U2 & N1_8 & $96.89(11)$ & C10_5 & N3_5 & C6_5 & $112.88(17)$ \\
\hline C16__10 & N5_10 & $\mathrm{U} 1^{-}$ & $165.6(4)$ & C11_5 & N4_5 & P1_5 & $123.21(14)$ \\
\hline N5_10 & C16_10 & C17_10 & $119.0(5)$ & C11_5 & N4_5 & C15_5 & $113.19(16)$ \\
\hline N5_10 & C16_10 & C21_10 & $122.1(5)$ & C15_5 & N4_5 & P1_ 5 & $119.33(13)$ \\
\hline C17̄_10 & C16_10 & C21_10 & $118.9(5)$ & N2_ 5 & C1_5 & C2_5 & $110.49(17)$ \\
\hline C16_10 & C17_10 & C22_10 & 118.6(5) & C1_5 & C2_5 & C3_5 & $110.87(18)$ \\
\hline C18_10 & C17_10 & C16_10 & $119.8(5)$ & $\mathrm{C} 2{ }^{-} 5$ & C3_5 & C4_5 & $110.02(18)$ \\
\hline C18_10 & C17_10 & C22_10 & $121.6(5)$ & C5_5 & C4_5 & C3_5 & $110.46(18)$ \\
\hline C17_10 & C18_10 & C19_10 & $121.9(5)$ & N2_5 & C5_5 & C4_5 & $110.58(17)$ \\
\hline C18_10 & C19_10 & C20_10 & $118.2(5)$ & N3_5 & C6_5 & C7_5 & $112.82(18)$ \\
\hline C18_10 & C19_10 & C23_10 & $121.7(5)$ & C6_5 & C7_5 & C8_5 & $112.1(2)$ \\
\hline C20_10 & C19_10 & C23_10 & $120.1(5)$ & C9_5 & C8_5 & C7_5 & $110.25(19)$ \\
\hline C21_10 & C20_10 & C19_10 & $121.9(5)$ & C10_5 & C9_5 & C8_5 & $111.8(2)$ \\
\hline C16_10 & C21_10 & C24_10 & $120.1(5)$ & N3_ $\overline{5}$ & C10_5 & C9_5 & $112.89(18)$ \\
\hline C20_10 & C21_10 & C16_10 & 119.3(5) & N4_5 & C11_5 & C12_5 & $112.85(17)$ \\
\hline C20_10 & C21_10 & C24_10 & $120.5(5)$ & C11__5 & C12_5 & C13_5 & $111.7(2)$ \\
\hline C16_11 & N5_- 11 & $\mathrm{U} 2^{-}$ & $165.5(4)$ & C12_5 & C13_5 & C14_5 & $110.04(19)$ \\
\hline N5_- 11 & C1̄̄_11 & C17_11 & $120.9(5)$ & C15_5 & C14_5 & C13_5 & $112.0(2)$ \\
\hline N5_11 & C16_11 & C21_11 & $120.0(4)$ & N4_-̄ & C15_5 & C14_5 & $112.85(17)$ \\
\hline C21__11 & C16_11 & C17_11 & 119.1(4) & N1_6 & $\mathrm{P} 1 \_\overline{6}$ & N2_- 6 & $119.44(10)$ \\
\hline C16_11 & C17_11 & C22_11 & $120.5(4)$ & N1_6 & P1_6 & N3_6 & $111.62(10)$ \\
\hline C18_11 & C17_11 & C16_11 & $118.8(5)$ & N1_6 & P1_6 & N4_6 & $112.00(10)$ \\
\hline C18_11 & C17_11 & C22_11 & $120.7(5)$ & N3_6 & P1_6 & N2_6 & $102.03(9)$ \\
\hline
\end{tabular}




\begin{tabular}{|c|c|c|c|c|c|c|c|}
\hline C17_11 & C18_11 & C19_11 & $122.1(5)$ & N4_6 & P1_6 & N2_6 & $101.63(9)$ \\
\hline C18_11 & C19_11 & C23_11 & $120.6(5)$ & N4_6 & P1_6 & N3_6 & $109.13(8)$ \\
\hline C20_11 & C19_11 & C18_11 & $118.4(5)$ & P1_6 & N1_6 & U2 & $154.10(15)$ \\
\hline C20_11 & C19_11 & C23_11 & $121.0(5)$ & C1_6 & N2_6 & P1_6 & $117.08(13)$ \\
\hline C19_11 & C20_11 & C21_11 & $122.3(5)$ & C1_6 & N2_6 & C5_6 & $111.53(16)$ \\
\hline C16_11 & C21_11 & C24_11 & 117.7(4) & C5_6 & N2_6 & P1_6 & $117.00(13)$ \\
\hline C20_11 & C21_11 & C16_11 & $119.2(5)$ & C6_6 & N3_6 & P1_6 & $120.67(14)$ \\
\hline C20_11 & C21_11 & C24_11 & $123.0(5)$ & C10_6 & N3_6 & P1_6 & $124.36(14)$ \\
\hline N1_ $\overline{1}$ & P1_ & N2 & $120.00(10)$ & C10_6 & N3_6 & C6_6 6 & $114.25(17)$ \\
\hline N1_1 & $\mathrm{P} 1 \_1$ & N3_1 & $111.98(10)$ & C11_6 & N4_6 & P1_6 & $124.52(14)$ \\
\hline N1_1 & P1_1 & N4_1 & 111.71(10) & C11_6 & N4_6 & C15_6 & $114.66(16)$ \\
\hline N2_1 & P1_1 & N4_1 & $101.22(9)$ & C15_6 & N4_6 & P1_6 & $120.55(14)$ \\
\hline N3_1 & P1_1 & N2_1 & $102.24(9)$ & N2_- 6 & C1_6 & C2_6 6 & $110.44(17)$ \\
\hline N3_-1 & P1_1 & N4_1 & 108.61(8) & C3_6 & $\mathrm{C} 2{ }^{-} 6$ & C1_6 & $110.82(18)$ \\
\hline P1_1 & N1_1 & U1 & $150.85(15)$ & C2_6 & C3_6 & C4_6 6 & $110.26(18)$ \\
\hline C1_1 & N2_1 & P1_1 & $117.54(13)$ & C5_6 & C4_6 & C3_6 & $110.38(18)$ \\
\hline C5_1 & N2_1 & P1_1 & 117.69(13) & N2_6 & C5_6 & C4_6 6 & $110.52(17)$ \\
\hline C5_1 & $\mathrm{N} 2{ }_{-}^{-} 1$ & C1_1 & 111.93(16) & N3_6 & C6_6 & C7_6 & $113.10(18)$ \\
\hline C6_1 & N3_1 & P1_1 & $121.06(14)$ & C6_6 & C7_6 & C8_6 6 & $112.0(2)$ \\
\hline C10_1 & N3_-1 & P1_1 & $124.49(14)$ & C9_6 & C8_6 & C7_6 & $110.41(19)$ \\
\hline C10-1 & N3_1 & C6_1 & $114.43(17)$ & C10_6 & C9_6 & C8_6 6 & $111.9(2)$ \\
\hline C11_1 & N4_1 & $\mathrm{P} 1 \_1$ & $122.57(14)$ & N3_ 6 & C10_6 & C9_6 & $113.23(18)$ \\
\hline C15_1 & N4_1 & P1_1 & $119.24(14)$ & N4_6 & C11_6 & C12 12 & $113.45(18)$ \\
\hline C15_1 & N4_1 & C11__1 & $113.55(17)$ & C11̄_6 & C12_6 & C13_6 & $111.7(2)$ \\
\hline N2_- $\overline{1}$ & $\mathrm{C}^{-1} 1$ & C2 $2 \overline{1}$ & $110.43(17)$ & C12_6 & C13_6 & C14_6 & $110.02(19)$ \\
\hline C3_1 & $\mathrm{C} 2{ }^{-} 1$ & C1_1 & $110.77(18)$ & C15_6 & C14_6 & C13_6 & $112.0(2)$ \\
\hline $\mathrm{C} 2{ }_{-1}$ & C3_1 & C4_1 & $110.36(18)$ & N4_- 6 & C15_6 & C14_6 & $113.41(18)$ \\
\hline C5_1 & C4_1 & C3_1 & $110.41(18)$ & N1_7 & P1_ 7 & $\mathrm{~N} 2 \_\overline{7}$ & $119.92(10)$ \\
\hline $\mathrm{N} 2{ }_{-1}$ & C5_1 & C4_1 & $110.61(17)$ & N1_7 & P1_7 & N3_7 & $112.04(10)$ \\
\hline N3_1 & C6_1 & C7_-1 & $113.23(18)$ & N1_7 & P1_7 & N4_7 & $111.64(10)$ \\
\hline C6_-1 & C7_-1 & C8_1 & $112.2(2)$ & $\mathrm{N} 2{ }^{-} 7$ & P1_7 & N4_7 & 101.60(9) \\
\hline C9_1 & C8_1 & C7_-1 & $110.51(19)$ & N3_7 & P1_7 & N2_7 & $102.04(9)$ \\
\hline C10_1 & C9_1 & C8_1 & $111.8(2)$ & N3_7 & P1_7 & N4_7 & 108.51(8) \\
\hline N3_ $\overline{1}$ & C10_1 & C9_1 & $113.11(18)$ & P1_7 & N1_7 & $\mathrm{U} 2^{-}$ & $173.41(15)$ \\
\hline N4_1 & C11_1 & C12̄_1 & $112.99(18)$ & C1_7 & $\mathrm{N} 2 \_7$ & P1_7 & $117.58(13)$ \\
\hline C11_1 & C12_1 & C13_1 & $111.6(2)$ & C1_7 & N2_7 & C5_7 & $111.40(16)$ \\
\hline C12_1 & C13-1 & C14_1 & $109.9(2)$ & C5_7 & N2_7 & P1_7 & $116.62(13)$ \\
\hline C15_1 & C14_1 & C13_1 & $112.0(2)$ & C6_7 & N3_7 & P1_7 & $120.99(14)$ \\
\hline N4_ & C15_1 & C14_1 & $113.26(18)$ & C10_7 & N3_7 & P1_7 & $124.52(14)$ \\
\hline N1_2 & P1_2 & N2_- & $120.64(10)$ & C10_7 & N3_7 & C6_7 & $114.47(17)$ \\
\hline N1_2 & P1_2 & N3_2 & 111.74(10) & C11_7 & N4_7 & P1_7 & $121.85(14)$ \\
\hline N1_2 & P1_2 & N4_2 & $111.62(9)$ & C11_7 & N4_7 & C15_7 & $112.69(17)$ \\
\hline $\mathrm{N} 2{ }^{-} 2$ & P1_2 & N3_2 & $102.11(8)$ & C15_7 & N4_7 & P1_ $\overline{7}$ & $117.91(13)$ \\
\hline N2_2 & P1_2 & N4_2 & $101.57(8)$ & $\mathrm{N} 2 \overline{7}$ & C1_7 & $\mathrm{C} 2 \_7$ & $110.49(17)$ \\
\hline N3_2 & P1_2 & N4_2 & 107.99(8) & C3_7 & $\mathrm{C} 2 \_7$ & C1_7 & $110.88(18)$ \\
\hline P1_2 & N1_2 & U1 & $170.48(15)$ & $\mathrm{C} 2{ }^{-} 7$ & C3_7 & C4_7 & $110.25(18)$ \\
\hline C1_2 & N2_2 & P1_2 & $118.65(13)$ & C5_7 & C4_7 & C3_7 & $110.40(18)$ \\
\hline C1_2 & N2_2 & C5_2 & $112.14(16)$ & N2_7 & C5_7 & C4_7 & $110.49(17)$ \\
\hline C5_2 & N2_2 & P1_2 & $118.10(13)$ & N3_7 & C6_7 & C7_7 & $113.13(18)$ \\
\hline
\end{tabular}




\begin{tabular}{|c|c|c|c|c|c|c|c|}
\hline C6_2 & N3_2 & P1_2 & $120.27(14)$ & C6_7 & C7_7 & C8_7 & $112.0(2)$ \\
\hline C6_2 & N3_2 & C10_2 & 113.61(17) & C9_7 & C8_7 & C7_-7 & $110.41(19)$ \\
\hline C10_2 & N3_2 & P1_2 & $123.30(14)$ & C10_7 & C9_7 & C8_7 & 111.8(2) \\
\hline C11_2 & N4_2 & P1_2 & $121.89(13)$ & N3_ $\overline{7}$ & C10_7 & C9_7 & $113.23(18)$ \\
\hline C15_2 & N4_2 & P1_2 & $118.29(13)$ & N4_7 & C11_7 & C12_7 & $113.01(18)$ \\
\hline C15_2 & N4_2 & C111_2 & $112.89(17)$ & C11̄_7 & C12_7 & C13_7 & $111.6(2)$ \\
\hline N2_- & C1_2 & C2_- & $110.46(17)$ & C12_7 & C13_7 & C14_7 & 109.62(19) \\
\hline C3_2 & C2_2 & C1_2 & $110.86(18)$ & C15_7 & C14_7 & C13_7 & $112.0(2)$ \\
\hline C2_2 & C3_2 & C4_2 & 110.53(18) & N4_ $\overline{7}$ & C15_7 & C14_7 & $113.21(18)$ \\
\hline C5_2 & C4_2 & C3_2 & $110.35(18)$ & N1_8 & P1_- 8 & N2_- 8 & $120.37(10)$ \\
\hline $\mathrm{N} 2-2$ & C5_2 & C4_2 & $110.47(17)$ & N1_8 & P1_8 & N3_8 & $111.26(10)$ \\
\hline N3_2 & C6_2 & C7_2 & $113.03(18)$ & N1_8 & P1_8 & N4_8 & $112.31(9)$ \\
\hline C6_2 & C7_2 & C8_2 & $112.2(2)$ & N2_8 & P1_8 & N3_8 & 101.86(8) \\
\hline C9_2 & C8_2 & C7_2 & $110.42(19)$ & N4_8 & P1_8 & N2_8 & 101.75(8) \\
\hline C10_2 & C9_2 & C8_2 & $111.6(2)$ & N4_8 & P1_8 & N3_8 & $108.13(8)$ \\
\hline N3_2 & C10_2 & C9_2 & $112.66(18)$ & P1_8 & N1_8 & U2 & $169.91(15)$ \\
\hline N4_2 & C11_2 & $\mathrm{C} 1 \overline{2} \_2$ & 112.93(18) & C1_8 & N2_8 & P1_8 & $117.65(13)$ \\
\hline C11__2 & C12_2 & C13_2 & $111.6(2)$ & C5_8 & N2_8 & P1_8 & $118.08(13)$ \\
\hline C12_2 & C13_2 & C14_2 & $109.77(19)$ & C5_8 & N2_8 & C1_8 & $111.81(16)$ \\
\hline C15_2 & C14_2 & C13_2 & $112.0(2)$ & C6_8 & N3_8 & P1_8 & $118.39(13)$ \\
\hline N4_ $\overline{2}$ & C15_2 & C14_2 & $113.06(18)$ & C10_8 & N3_8 & P1_8 & $121.84(14)$ \\
\hline N1_3 & P1_ 3 & N2_- 3 & $119.41(10)$ & C10_8 & N3_8 & C6_8 & $112.59(17)$ \\
\hline N1_3 & P1_3 & N3_3 & $111.79(10)$ & C11_8 & N4_8 & P1_8 & $123.92(14)$ \\
\hline N1_3 & P1_3 & N4_3 & $112.12(10)$ & C15_8 & N4_8 & P1_8 & $120.69(14)$ \\
\hline N3_3 & P1_3 & N2_3 & $102.10(9)$ & C15_8 & N4_8 & C11̄_8 & $114.45(16)$ \\
\hline N4_3 & P1_3 & N2_3 & $101.44(9)$ & N2_- 8 & C1_8 & C2_- 8 & $110.29(17)$ \\
\hline N4_3 & P1_3 & N3_3 & $108.94(9)$ & C3_8 & C2_8 & C1_8 & $110.74(18)$ \\
\hline P1_3 & N1_3 & $\mathrm{U} 1^{-}$ & $158.8(2)$ & $\mathrm{C} 2{ }^{-} 8$ & C3_8 & C4_8 & $110.45(18)$ \\
\hline C1_3 & N2_3 & P1_3 & $116.60(14)$ & C5_8 & C4_8 & C3_8 & $110.48(18)$ \\
\hline C5_3 & N2_3 & P1_3 & $116.90(14)$ & N2_8 & C5_8 & C4_8 & $110.56(17)$ \\
\hline C5_3 & N2_3 & C1_3 & $111.36(17)$ & N3_8 & C6_8 & C7_8 & $112.82(18)$ \\
\hline C6_3 & N3_3 & P1_3 & $120.36(14)$ & C6_8 & C7_8 & C8_8 & $112.1(2)$ \\
\hline C10_3 & N3_3 & P1_3 & $123.99(15)$ & C9_8 & C8_8 & C7_8 & $110.15(19)$ \\
\hline C10_3 & N3_3 & C6_3 & $113.86(17)$ & C10_8 & C9_8 & C8_8 & $111.8(2)$ \\
\hline C11_3 & N4_3 & P1_3 & $123.87(14)$ & N3_ $\overline{8}$ & C10_8 & C9_8 & $112.85(18)$ \\
\hline C15_3 & N4_3 & P1_3 & $120.24(14)$ & N4_8 & C11_8 & C12_8 & $113.03(18)$ \\
\hline C15_3 & N4_3 & C11__3 & $114.19(17)$ & C11̄_8 & C12_8 & C13_8 & $111.5(2)$ \\
\hline N2_ 3 & C1_3 & C2_3 & $110.41(17)$ & C12_8 & C13_8 & C14_8 & $109.99(19)$ \\
\hline C3_3 & C2_3 & C1_3 & $110.79(18)$ & C15_8 & C14_8 & C13_8 & $112.1(2)$ \\
\hline C2_3 & C3_3 & C4_3 & $110.22(19)$ & N4_-8 & C15_8 & C14_8 & $113.52(18)$ \\
\hline C5_3 & C4_3 & C3_3 & $110.47(18)$ & N1_9 & P1_̄̄ & N2__9 & $119.31(10)$ \\
\hline N2_3 & C5_3 & C4_3 & $110.62(17)$ & N1_9 & P1_9 & N3_9 & $111.81(10)$ \\
\hline N3_3 & C6_3 & C7_3 & $113.10(19)$ & N1_9 & P1_9 & N4_9 & $112.28(10)$ \\
\hline C6_3 & C7_3 & C8_3 & $112.1(2)$ & N3_9 & P1_9 & N2_9 & 102.01(9) \\
\hline C9_3 & C8_3 & C7_3 & $110.4(2)$ & N4_9 & P1_9 & N2_9 & $101.44(8)$ \\
\hline C10_3 & C9_3 & C8_3 & $111.8(2)$ & N4_9 & P1_9 & N3_9 & 108.93(8) \\
\hline N3_ $\overline{3}$ & C10_3 & C9_3 & $113.09(19)$ & P1_9 & N1_9 & $\mathrm{U} 2^{-}$ & $165.09(16)$ \\
\hline N4_3 & C11_3 & C12 3 & $113.12(18)$ & C1_9 & N2_9 & P1_9 & $115.99(13)$ \\
\hline C111_3 & C12_3 & C13_3 & $111.6(2)$ & C5_9 & N2_9 & P1_9 & 116.34(13) \\
\hline
\end{tabular}




$\begin{array}{llllllll}\text { C12_3 } & \text { C13_3 } & \text { C14_3 } & 110.0(2) & \text { C5_9 } & \text { N2_9 } & \text { C1_9 } & 110.94(16) \\ \text { C15_3 } & \text { C14_3 } & \text { C13_3 } & 112.0(2) & \text { C6_9 } & \text { N3_9 } & \text { P1_9 } & 119.45(14) \\ \text { N4_3 } & \text { C15_3 } & \text { C14_3 } & 113.30(19) & \text { C10_9 } & \text { N3_9 } & \text { P1_9 } & 123.13(14) \\ \text { N1_4 } & \text { P1_4 } & \text { N2_4 } & 119.28(10) & \text { C10_9 } & \text { N3_9 } & \text { C6_9 } & 112.68(17) \\ \text { N1_4 } & \text { P1_4 } & \text { N3_4 } & 111.82(10) & \text { C11_9 } & \text { N4_9 } & \text { P1_9 } & 123.52(14) \\ \text { N1_4 } & \text { P1_4 } & \text { N4_4 } & 112.06(10) & \text { C15_9 } & \text { N4_9 } & \text { P1_9 } & 119.90(13) \\ \text { N3_4 } & \text { P1_4 } & \text { N2_4 } & 102.15(9) & \text { C15_9 } & \text { N4_9 } & \text { C11_9 } & 113.73(17) \\ \text { N4_4 } & \text { P1_4 } & \text { N2_4 } & 101.43(9) & \text { N2_9 } & \text { C1_9 } & \text { C2_9 } & 110.38(17) \\ \text { N4_4 } & \text { P1_4 } & \text { N3_4 } & 109.09(9) & \text { C1_9 } & \text { C2_9 } & \text { C3_9 } & 110.79(18) \\ \text { P1_4 } & \text { N1_4 } & \text { U1 } & 177.0(3) & \text { C2_9 } & \text { C3_9 } & \text { C4_9 } & 110.08(18) \\ \text { C1_4 } & \text { N2_4 } & \text { P1_4 } & 116.64(14) & \text { C5_9 } & \text { C4_9 } & \text { C3_9 } & 110.49(18) \\ \text { C5_4 } & \text { N2_4 } & \text { P1_4 } & 116.90(14) & \text { N2_9 } & \text { C5_9 } & \text { C4_9 } & 110.63(17) \\ \text { C5_4 } & \text { N2_4 } & \text { C1_4 } & 111.40(17) & \text { N3_9 } & \text { C6_9 } & \text { C7_9 } & 112.40(17) \\ \text { C6_4 } & \text { N3_4 } & \text { P1_4 } & 120.83(14) & \text { C6_9 } & \text { C7_9 } & \text { C8_9 } & 112.0(2) \\ \text { C10_4 } & \text { N3_4 } & \text { P1_4 } & 124.57(15) & \text { C9_9 } & \text { C8_9 } & \text { C7_9 } & 110.49(19) \\ \text { C10_4 } & \text { N3_4 } & \text { C6_4 } & 114.40(17) & \text { C10_9 } & \text { C9_9 } & \text { C8_9 } & 111.9(2) \\ \text { C11_4 } & \text { N4_4 } & \text { P1_4 } & 124.12(14) & \text { N3_9 } & \text { C10_9 } & \text { C9_9 } & 112.56(17) \\ \text { C15_4 } & \text { N4_4 } & \text { P1_4 } & 120.45(14) & \text { N4_9 } & \text { C11_9 } & \text { C12_9 } & 112.95(18) \\ \text { C15_4 } & \text { N4_4 } & \text { C11_4 } & 114.50(17) & \text { C11_9 } & \text { C12_9 } & \text { C13_9 } & 111.5(2) \\ \text { N2_4 } & \text { C1_4 } & \text { C2_4 } & 110.41(17) & \text { C12_9 } & \text { C13_9 } & \text { C14_9 } & 109.95(19) \\ \text { C3_4 } & \text { C2_4 } & \text { C1_4 } & 110.79(18) & \text { C15_9 } & \text { C14_9 } & \text { C13_9 } & 112.1(2) \\ \text { C2_4 } & \text { C3_4 } & \text { C4_4 } & 110.23(19) & \text { N4_9 } & \text { C15_9 } & \text { C14_9 } & 113.27(18) \\ \text { C5_4 } & \text { C4_4 } & \text { C3_4 } & 110.46(18) & & & & \end{array}$

Table S6: Bond Lengths in $\AA$ for 2-U(PN)O.

\begin{tabular}{lll}
\hline Atom & Atom & Length/A \\
\hline N3 & U1 & $1.948(7)$ \\
N3 & P2 & $1.530(4)$ \\
N2 & U1 & $2.161(18)$ \\
N2 & P1 & $1.533(18)$ \\
O1 & U1 & $1.98(3)$ \\
P1 & N1_1 & $1.685(6)$ \\
P1 & N1_2 & $1.695(6)$ \\
P1 & N1_3 & $1.684(7)$ \\
P2 & N1_41 & $1.622(11)$ \\
P2 & N1_4 & $1.622(11)$ \\
P2 & N1_4 & $1.622(11)$ \\
N1_1 & C2_1 & $1.454(9)$ \\
N1_1 & C6_1 & $1.454(9)$ \\
C2_1 & C3_1 & $1.472(9)$ \\
C3_1 & C4_1 & $1.472(9)$ \\
C4_1 & C5_1 & $1.473(9)$ \\
C5_1 & C6_1 & $1.473(9)$ \\
N1_2 & C2_2 & $1.455(9)$ \\
N1_2 & C6_2 & $1.455(9)$ \\
C2_2 & C3_2 & $1.473(9)$ \\
C3_2 & C4_2 & $1.473(9)$ \\
C4_2 & C5_2 & $1.473(9)$ \\
C5_2 & C6_2 & $1.473(9)$
\end{tabular}




\begin{tabular}{lll}
\hline Atom & Atom & Length/A \\
\hline N1_3 & C2_3 & $1.455(9)$ \\
N1_3 & C6_3 & $1.454(9)$ \\
C2_3 & C3_3 & $1.473(9)$ \\
C3_3 & C4_3 & $1.473(9)$ \\
C4_3 & C5_3 & $1.473(9)$ \\
C5_3 & C6_3 & $1.472(9)$ \\
N1_4 & C2_4 & $1.455(9)$ \\
N1_4 & C6_4 & $1.455(9)$ \\
C2_4 & C3_4 & $1.473(9)$ \\
C3_4 & C4_4 & $1.473(9)$ \\
C4_4 & C5_4 & $1.473(9)$ \\
C5_4 & C6_4 & $1.473(9)$ \\
1+y-x,1-x, +z; ${ }^{21-y, 1+x-y,+z}$
\end{tabular}

Table S7: Bond Angles in ${ }^{\circ}$ for 2-U(PN)O.

\begin{tabular}{llll}
\hline Atom & Atom & Atom & \multicolumn{1}{c}{ Anglel $^{\circ}$} \\
\hline P2 & N3 & U1 & 180.0 \\
P1 & N2 & U1 & $144.9(12)$ \\
N3 & U1 & N2 & $94.8(5)$ \\
N3 & U1 & N2 ${ }^{2}$ & $94.8(5)$ \\
N3 & U1 & N2 & $94.8(5)$ \\
N3 & U1 & O1 & $180.000(1$ \\
N21 & U1 & N2 & $1)$ \\
N2 & U1 & N21 & $119.32(14)$ \\
N2 & U1 & N2 & $119.32(14)$ \\
O1 & U1 & N2 & $85.2(5)$ \\
O1 & U1 & N2 & $85.2(5)$ \\
O1 & U1 & N21 & $85.2(5)$ \\
N2 & P1 & N1_1 & $121.2(8)$ \\
N2 & P1 & N1_2 & $116.9(8)$ \\
N2 & P1 & N1_3 & $102.7(9)$ \\
N1_1 & P1 & N1_2 & $100.3(6)$ \\
N1_3 & P1 & N1_1 & $107.0(8)$ \\
N1_3 & P1 & N1_2 & $108.1(8)$ \\
N3 & P2 & N1_4 & $113.8(5)$ \\
N3 & P2 & N1_4 & $113.8(5)$ \\
N3 & P2 & N1_4 1 & $113.8(5)$ \\
N1_4 & P2 & N1_41 & $104.9(6)$ \\
N1_4 & P2 & N1_41 & $104.9(6)$ \\
N1_4 & P2 & N1_4 & $104.9(6)$ \\
C2_1 & N1_1 & P1 & $113.8(8)$ \\
C2_1 & N1_1 & C6_1 & $109.6(8)$ \\
C6_1 & N1_1 & P1 & $126.0(9)$ \\
N1_1 & C2_1 & C3_1 & $108.8(9)$ \\
C4_1 & C3_1 & C2_1 & $111.3(12)$ \\
C3_1 & C4_1 & C5_1 & $109.4(11)$ \\
C4_1 & C5_1 & C6_1 & $115.8(12)$ \\
N1_1 & C6_1 & C5_1 & $106.2(9)$ \\
C2_2 & N1_2 & P1 & $111.4(9)$ \\
C2_2 & N1_2 & C6_2 & $109.5(8)$ \\
C6_2 & N1_2 & P1 & $115.2(9)$ \\
& & &
\end{tabular}




\begin{tabular}{llll}
\hline Atom & Atom & Atom & \multicolumn{1}{c}{ Angle $^{\circ}$} \\
\hline N1_2 & C2_2 & C3_2 & $108.8(9)$ \\
C4_2 & C3_2 & C2_2 & $111.2(12)$ \\
C3_2 & C4_2 & C5_2 & $109.3(11)$ \\
C6_2 & C5_2 & C4_2 & $115.8(12)$ \\
N1_2 & C6_2 & C5_2 & $106.2(8)$ \\
C2_3 & N1_3 & P1 & $124.6(11)$ \\
C6_3 & N1_3 & P1 & $123.4(11)$ \\
C6_3 & N1_3 & C2_3 & $109.7(8)$ \\
N1_3 & C2_3 & C3_3 & $108.7(9)$ \\
C4_3 & C3_3 & C2_3 & $111.1(12)$ \\
C5_3 & C4_3 & C3_3 & $109.3(11)$ \\
C6_3 & C5_3 & C4_3 & $115.9(12)$ \\
N1133 & C6_3 & C5_3 & $106.3(9)$ \\
C2_4 & N1_4 & P2 & $128.0(10)$ \\
C6_4 & N1_4 & P2 & $116.8(9)$ \\
C6_4 & N1_4 & C2_4 & $109.5(8)$ \\
N1_4 & C2_4 & C3_4 & $108.7(9)$ \\
C2_4 & C3_4 & C4_4 & $111.2(12)$ \\
C3_4 & C4_4 & C5_4 & $109.3(11)$ \\
C6_4 & C5_4 & C4_4 & $115.8(12)$ \\
N1_4 & C6_4 & C5_4 & $106.2(9)$ \\
---- & & &
\end{tabular}


UV/vis/NIR electronic absorption spectra

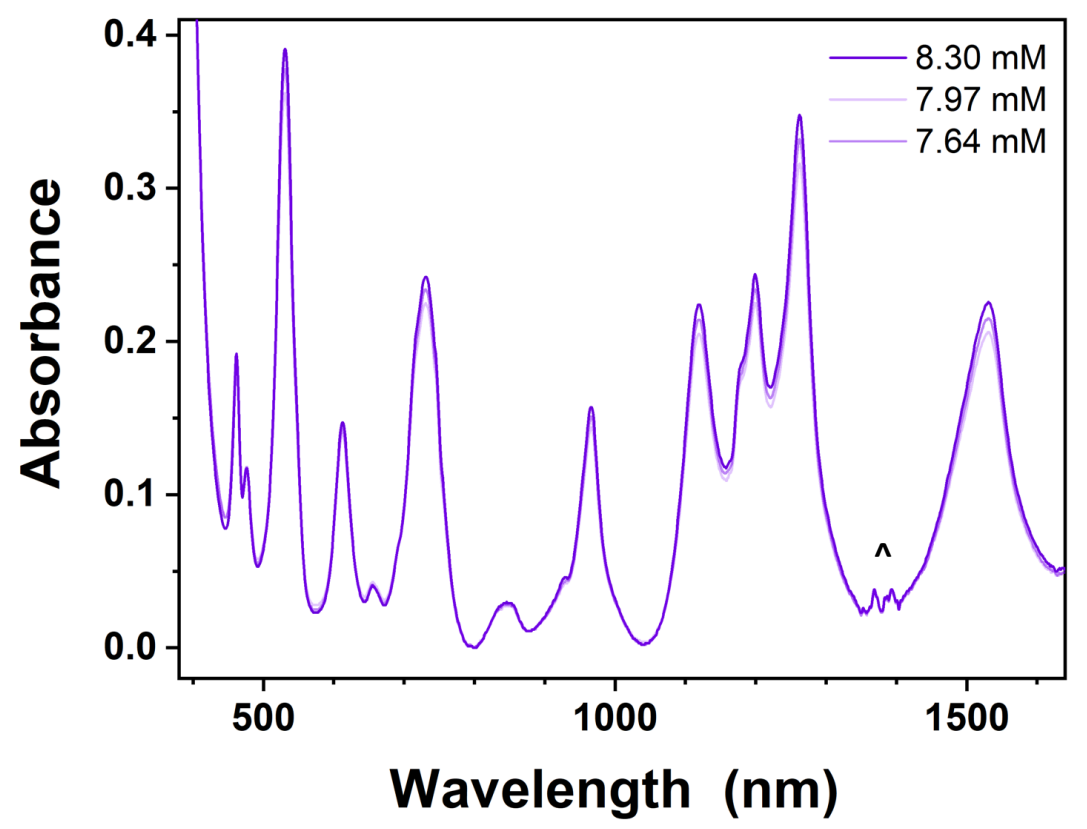

Figure S22. Concentration dependent UV/vis/NIR spectra of 1-U(PN) in THF. Artifact is noted by ${ }^{\wedge}$. Observable transitions and their molar absorptivities are reported in Table S8 below.

Table S8. Concentration, Absorbance, and Molar Absorptivity values for observable transitions in concentrated samples of 1-U(PN). Absorbance values for transitions at 476 and $462 \mathrm{~nm}$ were too low to provide a reliable extinction coefficient value.

\begin{tabular}{|c|c|c|c|}
\hline Wavelength & $\begin{array}{l}\text { Concentration } \\
\text { (M) }\end{array}$ & Absorbance & $\begin{array}{l}\text { Molar absorptivity } \\
\left(\mathrm{cm}^{-1} \mathrm{M}^{-1}\right)\end{array}$ \\
\hline \multirow[t]{3}{*}{1531} & 8.30E-03 & 0.226 & $\varepsilon=30$ \\
\hline & 7.97E-03 & 0.215 & \\
\hline & 7.64E-03 & 0.206 & \\
\hline \multirow[t]{3}{*}{1262} & 8.30E-03 & 0.348 & $\varepsilon=30$ \\
\hline & 7.97E-03 & 0.332 & \\
\hline & 7.64E-03 & 0.316 & \\
\hline \multirow[t]{3}{*}{1199} & 8.30E-03 & 0.244 & $\varepsilon=29$ \\
\hline & 7.97E-03 & 0.234 & \\
\hline & 7.64E-03 & 0.225 & \\
\hline \multirow[t]{3}{*}{1120} & 8.30E-03 & 0.224 & $\varepsilon=29$ \\
\hline & 7.97E-03 & 0.214 & \\
\hline & $7.64 \mathrm{E}-03$ & 0.205 & \\
\hline \multirow[t]{3}{*}{966} & $8.30 \mathrm{E}-03$ & 0.157 & $\varepsilon=20$ \\
\hline & 7.97E-03 & 0.151 & \\
\hline & 7.64E-03 & 0.144 & \\
\hline
\end{tabular}




\begin{tabular}{l|rrr}
731 & $8.30 \mathrm{E}-03$ & 0.242 & $\varepsilon=26$ \\
& $7.97 \mathrm{E}-03$ & 0.234 & \\
& $7.64 \mathrm{E}-03$ & 0.225 & \\
& $8.30 \mathrm{E}-03$ & 0.147 & $\varepsilon=11$ \\
& $7.97 \mathrm{E}-03$ & 0.143 & \\
& $7.64 \mathrm{E}-03$ & 0.14 & \\
& $8.30 \mathrm{E}-03$ & 0.391 & $\varepsilon=44$ \\
& $7.97 \mathrm{E}-03$ & 0.378 & \\
& $7.64 \mathrm{E}-03$ & 0.362 & \\
& $8.30 \mathrm{E}-03$ & 0.117 & $\mathrm{NA}$ \\
& $7.97 \mathrm{E}-03$ & 0.118 & \\
& $7.64 \mathrm{E}-03$ & 0.119 & \\
& $8.30 \mathrm{E}-03$ & 0.192 & $\mathrm{NA}$ \\
& $7.97 \mathrm{E}-03$ & 0.192 & \\
& $7.64 \mathrm{E}-03$ & 0.186 &
\end{tabular}
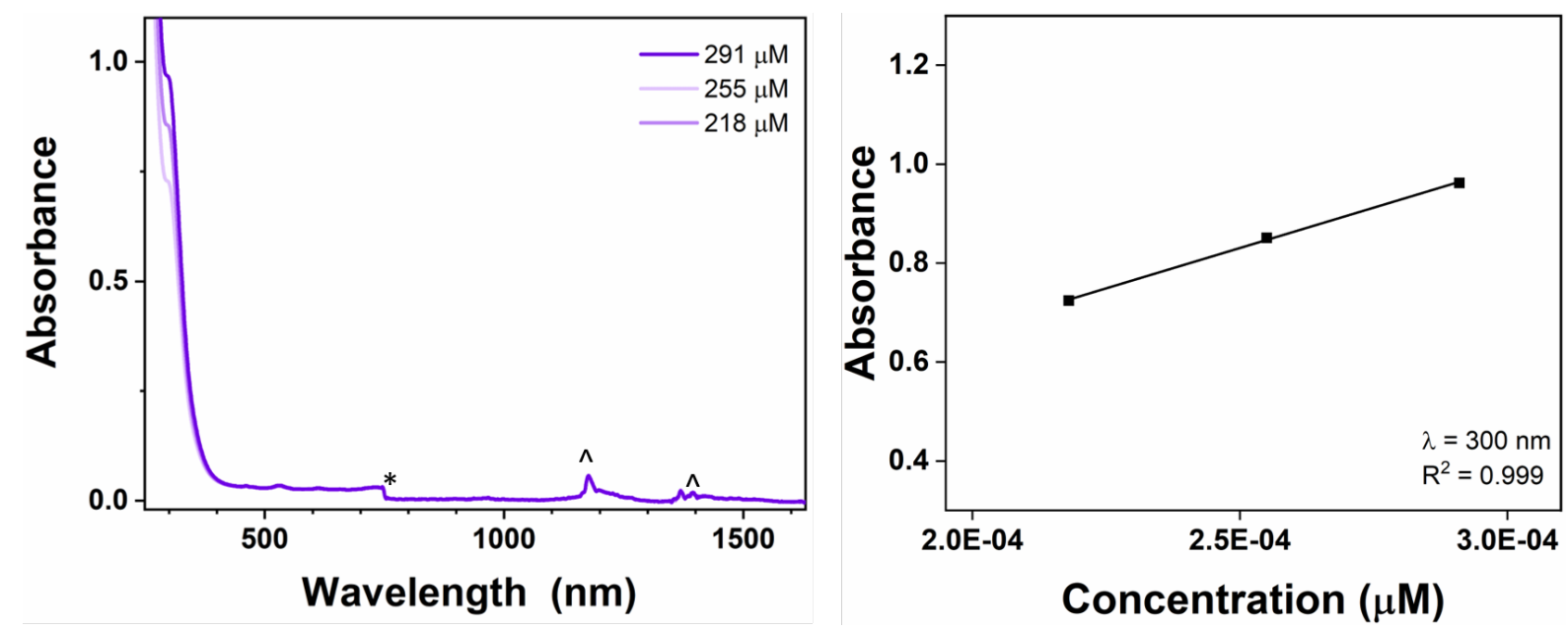

Figure S23. (left) Dilute UV/vis/NIR spectra of 1-U(PN) in THF. Grating change is denoted by ${ }^{*}$ and artifacts are noted by ${ }^{\wedge}$. (right) Linear regression of absorbance at 300 nm where $\varepsilon=3260 \mathrm{~cm}^{-1} \mathrm{M}^{-1}$. 

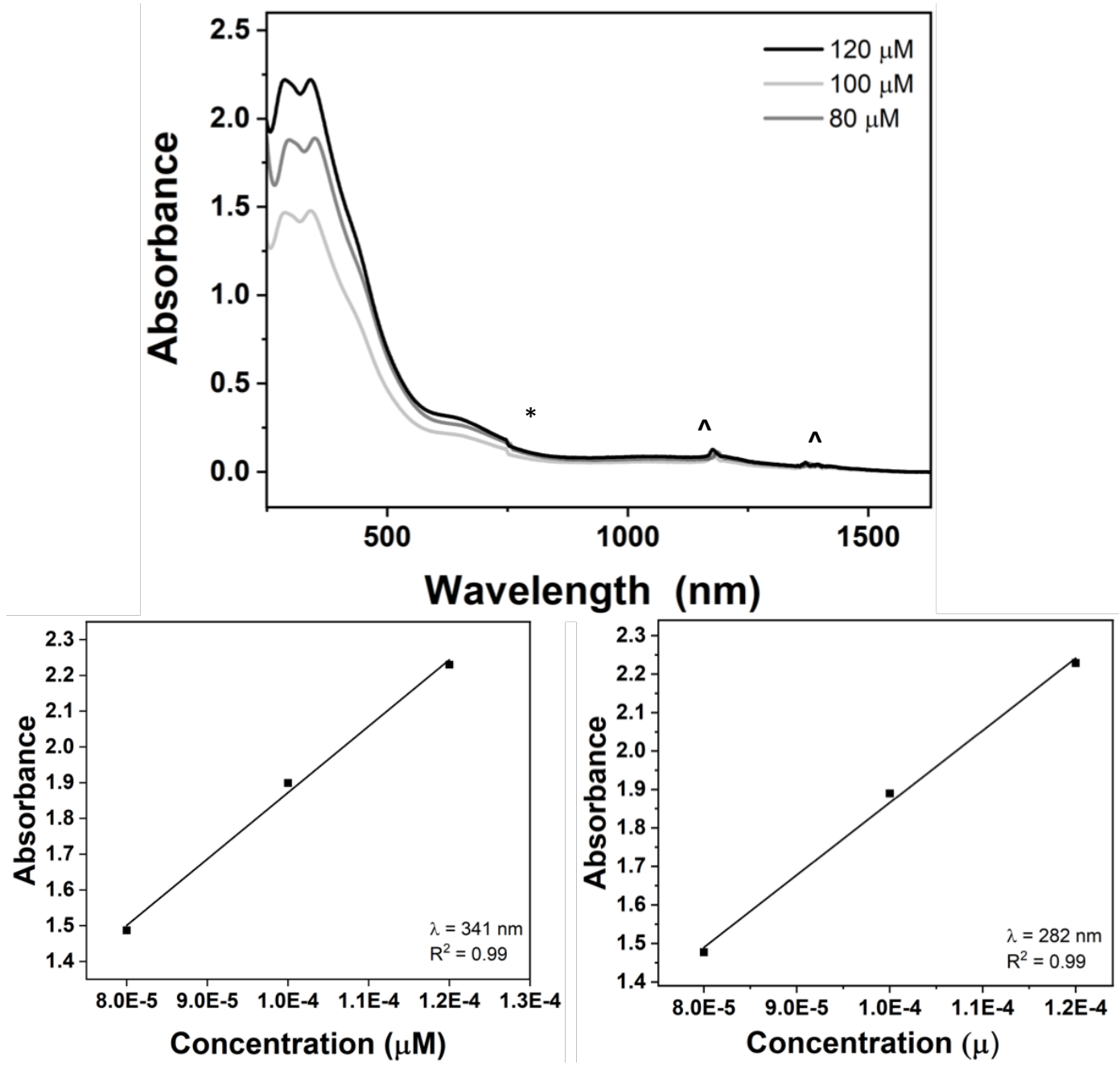

Figure S24. (top) UV/vis/NIR spectrum for 2-U(PN)NMes. Grating change is denoted by ${ }^{*}$ and artifacts are noted by ${ }^{\wedge}$. (bottom left) Linear regression of absorbance at $341 \mathrm{~nm}$ where $\varepsilon=18,600 \mathrm{~cm}^{-1} \mathrm{M}^{-1}$. (bottom right) Linear regression of absorbance at $282 \mathrm{~nm}$ where $\varepsilon=18,800 \mathrm{~cm}^{-1} \mathrm{M}^{-1}$. 

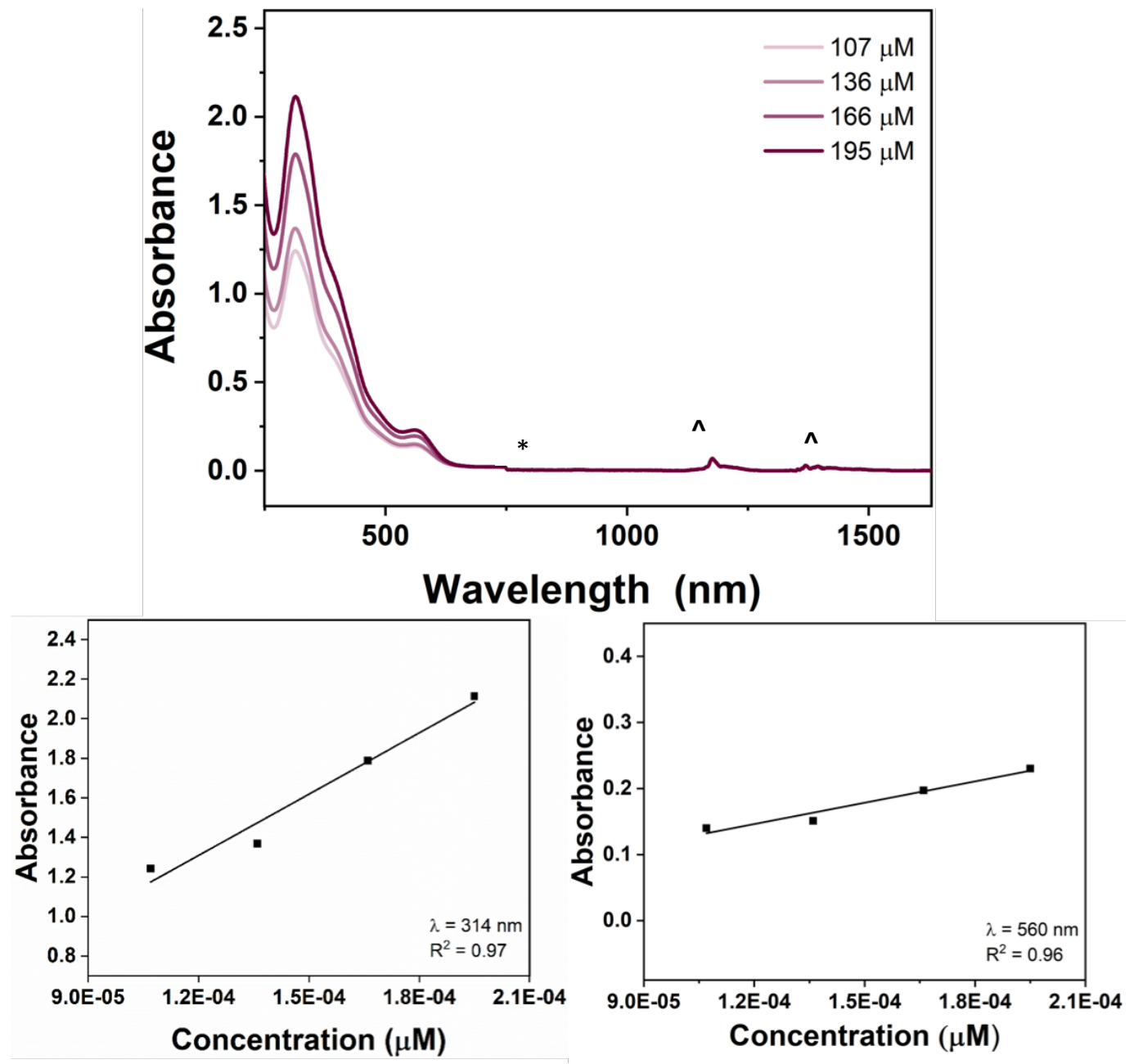

Figure S25. (top) UV/vis/NIR spectrum for 2-U(PN)O. Grating change is denoted by * and artifacts are noted by ${ }^{\wedge}$. (bottom left) Linear regression of absorbance at $560 \mathrm{~nm}$ where $\varepsilon=1,070 \mathrm{~cm}^{-1} \mathrm{M}^{-1}$. (bottom right) Linear regression of absorbance at $314 \mathrm{~nm}$ where $\varepsilon=10,300 \mathrm{~cm}^{-1} \mathrm{M}^{-1}$.

\section{Theoretical Calculations}

The calculations were conducted using a DFT level - using the Becke's 3-parameter hybrid functional combined with the non-local correlation functions designed by Perdew/Yang (B3PW91). ${ }^{8}, 9$ All calculations were computed using the Gaussian09 software. ${ }^{10}$ The Uranium and Phosphorus atoms were treated with a small-core Stuttgart Dresden relativistic effective core potential (SDDALL) $)^{11-13}$ in combination with a double quality basis set - SDDALL. ${ }^{14}$ Additionally, phosphorus atoms were treated with an adapted basis set. ${ }^{15-18}$ Carbon, Hydrogen and Nitrogen atoms were described using a double $\zeta 6-31 \mathrm{G}^{* *}$ basis set including $\mathrm{d}$ and $\mathrm{p}$ polarization respectively. ${ }^{19}$ Geometry optimizations of the various structures were computed without symmetry constraints. Furthermore, frequency analyses enabled the computation of force constants and 
vibrational frequencies on the system. Natural Bonding Orbital analyses enabled deeper understanding of the nature of the bonding within these systems. ${ }^{20}$

Table S9. Geometry optimized coordinates for 2-U(PN)NMes.

\begin{tabular}{|c|c|c|c|}
\hline & 12032000 & 4.035433000 & \\
\hline & 0.317490000 & 4.454077000 & \\
\hline & 3823000 & 891850000 & \\
\hline & 9663000 & 348131000 & \\
\hline & 2.141133000 & 931838000 & \\
\hline & 2.297224000 & 31440000 & \\
\hline & -0.766656000 & & \\
\hline & -0.333643000 & 1.979287000 & \\
\hline & 073411000 & -0.148630000 & -0.3202510 \\
\hline & -2.042094000 & 13836000 & \\
\hline & 276000 & -1.1 & \\
\hline & 42656000 & -2.8 & \\
\hline & 69267000 & 1000 & \\
\hline & 590000 & 56000 & \\
\hline & & & \\
\hline & -4.3 & & \\
\hline & 066000 & -3.8 & -0.255278 \\
\hline & -2.2 & 4000 & 0.431828 \\
\hline & & & \\
\hline & & & \\
\hline & -4.7 & & \\
\hline & -3.4 & 4.7 & \\
\hline & & & \\
\hline & & & \\
\hline & & & \\
\hline & -0.7 & 3.7 & \\
\hline & -1.1 & 5.2 & \\
\hline & & & \\
\hline & & & \\
\hline & 0.32 & $-0 . c$ & 1.6 \\
\hline & 0.35 & & 3.2 \\
\hline & & & \\
\hline & & & \\
\hline & & & \\
\hline & 3.98 & -3.8 & 4.4 \\
\hline & 3.3542080 & $-2.8 \varepsilon$ & 5.437228 \\
\hline & & & \\
\hline & & & \\
\hline & & & \\
\hline & & & \\
\hline & & & \\
\hline
\end{tabular}




\begin{tabular}{|c|c|c|c|}
\hline C & 0.494927000 & -2.503884000 & -2.644888000 \\
\hline C & 0.503119000 & -2.132884000 & -4.021495000 \\
\hline C & 0.694701000 & -3.106167000 & -4.998704000 \\
\hline C & 0.885130000 & -4.456623000 & -4.684503000 \\
\hline C & 0.877848000 & -4.809890000 & -3.332202000 \\
\hline C & 0.683253000 & -3.876135000 & -2.3132 \\
\hline C & 0.322207000 & -0.689638000 & -4.3941 \\
\hline C & 1.058080000 & -5.491390000 & -5.76 \\
\hline C & 0.641684000 & -4.311513000 & -0.8762 \\
\hline $\mathrm{N}$ & 0.307532000 & 0.018230000 & 4.3848 \\
\hline C & 1.510605000 & 0.838671000 & \\
\hline C & 1.442839000 & 1.592611000 & $5.878 \varepsilon$ \\
\hline C & 1000 & & \\
\hline C & -1.063130000 & 1.609646000 & 5.653 \\
\hline C & 7000 & 0.85 & \\
\hline$N$ & 896000 & 7184000 & 3.7 \\
\hline C & & -2.95 & \\
\hline C & -1.862384000 & 03000 & 3.1 \\
\hline C & -2.457551000 & -4.57 & 4.5 \\
\hline C & 000 & -3.7 & \\
\hline C & -1.50 & -2.2 & 5.0 \\
\hline $\mathrm{N}$ & 4.27 & -1.3 & -1.7 \\
\hline C & 4.02228 & -1.56 & -3.1 \\
\hline C & 5.0773 & -2.5 & -3.7 \\
\hline C & 5.079 & -3.8 & -2.9 \\
\hline C & 5.22 & & -1.4 \\
\hline C & 4.172724000 & -2.57 & -0. \\
\hline $\mathrm{N}$ & 4.197822000 & 1.25 & -2.1 \\
\hline C & 000 & 2.56 & -2.08 \\
\hline C & 3.684329000 & 3.265417000 & -3.4401 \\
\hline C & 5.14 & 3.36 & -3.8 \\
\hline C & 5.80606 & 1.99 & -3.84 \\
\hline C & 5.615 & 1.3 & -2.47 \\
\hline $\mathrm{N}$ & -4.55066 & $-1.0 \varsigma$ & 0.5 \\
\hline C & -5.95910 & -1.35 & 0.2 \\
\hline C & -6.680639000 & -1.73 & 1.5757 \\
\hline C & -6.528075000 & -0.61 & 2.5965 \\
\hline C & -5.049359000 & -0.271442000 & 2.7840 \\
\hline C & -4.379338000 & 0.023747000 & \\
\hline $\mathrm{N}$ & -4.146468000 & -0.367481000 & -2.1841 \\
\hline C & -3.507402000 & 0.793640000 & -2.78936 \\
\hline C & -4.547859000 & 1.854353000 & -3.15717900 \\
\hline C & -5.639663000 & 1.276314000 & -4.05806400 \\
\hline C & -6.258007000 & 0.040979000 & -3.40366600 \\
\hline C & -5.169621000 & -0.967482000 & -3.03335 \\
\hline $\mathrm{H}$ & 1.022573000 & -5.854708000 & -3.05765 \\
\hline
\end{tabular}




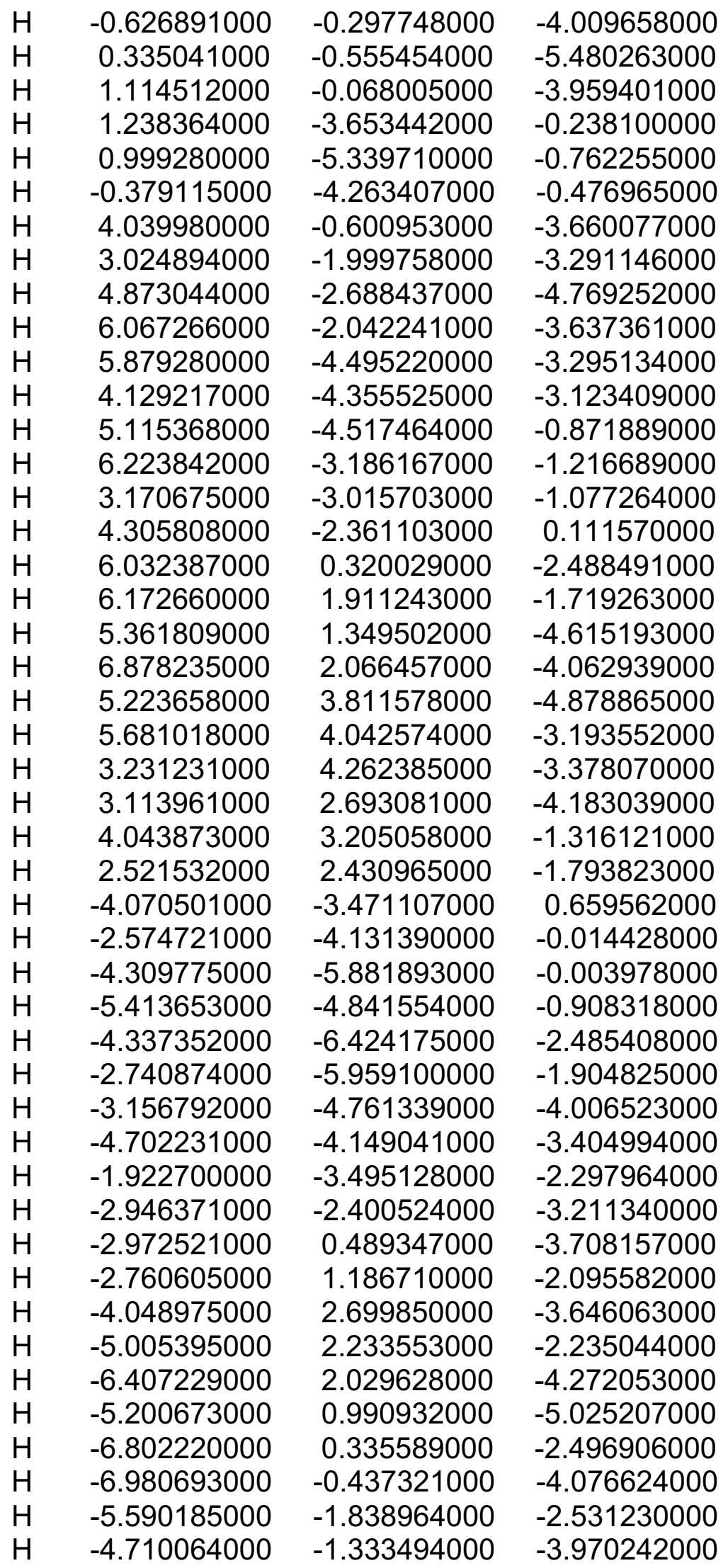




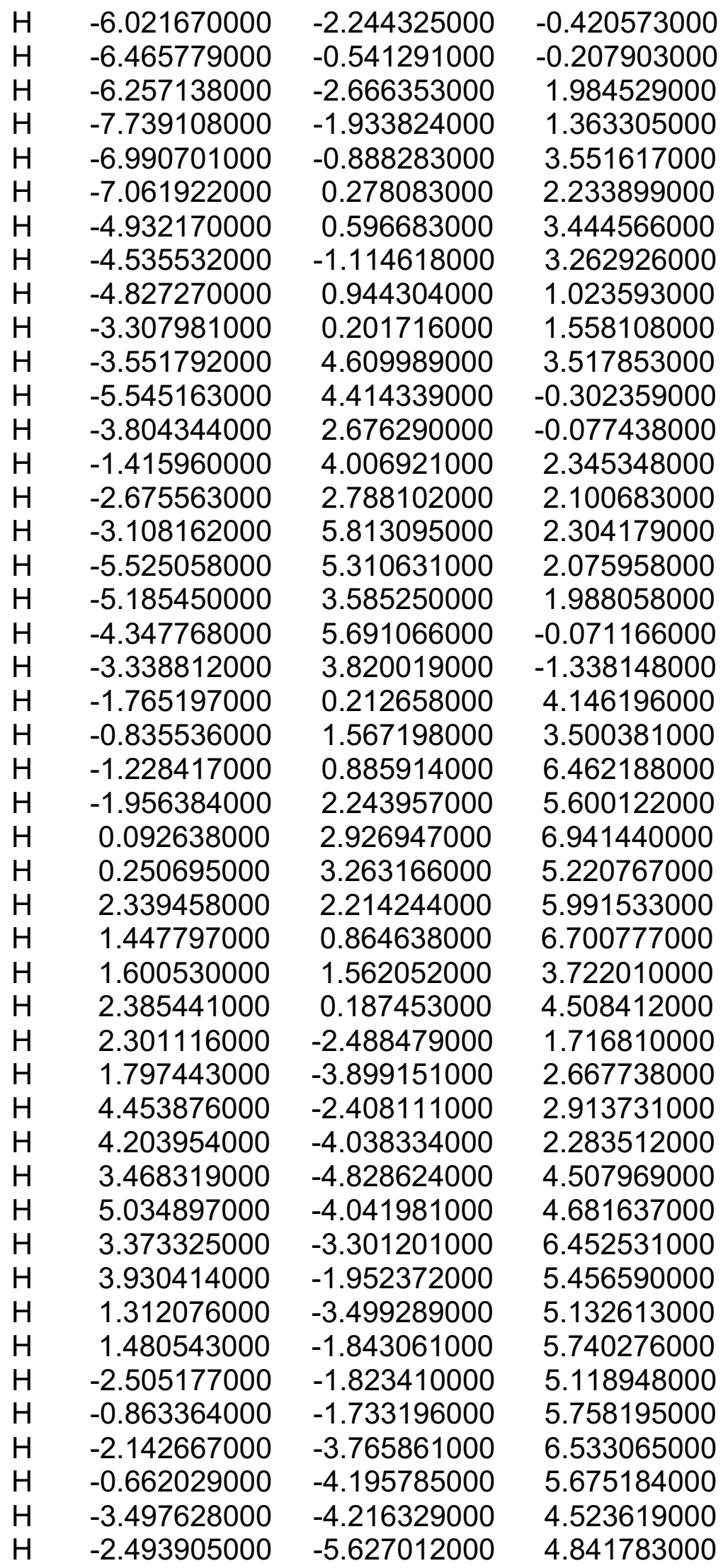




$\begin{array}{rrrr}\mathrm{H} & -0.862232000 & -4.882638000 & 3.106820000 \\ \mathrm{H} & -2.478442000 & -4.962827000 & 2.401059000 \\ \mathrm{H} & -1.289933000 & -2.818489000 & 1.765758000 \\ \mathrm{H} & -2.769176000 & -2.527736000 & 2.669205000 \\ \mathrm{H} & 0.844392000 & 4.128023000 & -2.952478000 \\ \mathrm{H} & 0.075318000 & 2.551420000 & -2.706918000 \\ \mathrm{H} & -0.095740000 & 3.366505000 & -5.078083000 \\ \mathrm{H} & -1.680964000 & 3.118046000 & -4.343151000 \\ \mathrm{H} & -0.234524000 & 5.799127000 & -4.676540000 \\ \mathrm{H} & -1.682533000 & 5.295606000 & -5.543181000 \\ \mathrm{H} & -2.200747000 & 6.825863000 & -3.586280000 \\ \mathrm{H} & -2.973613000 & 5.246397000 & -3.423141000 \\ \mathrm{H} & -0.387333000 & 6.168049000 & -2.077408000 \\ \mathrm{H} & -1.945956000 & 5.894196000 & -1.281445000 \\ \mathrm{H} & 0.657389000 & 6.453520000 & 0.045734000 \\ \mathrm{H} & -0.930566000 & 6.122712000 & 0.762537000 \\ \mathrm{H} & 0.570862000 & 7.436475000 & 2.281705000 \\ \mathrm{H} & 0.075834000 & 5.899165000 & 2.995275000 \\ \mathrm{H} & 2.757847000 & 6.485532000 & 1.638036000 \\ \mathrm{H} & 2.510705000 & 6.201282000 & 3.357877000 \\ \mathrm{H} & 3.353091000 & 4.142746000 & 2.149181000 \\ \mathrm{H} & 1.776252000 & 3.869107000 & 2.894579000 \\ \mathrm{H} & 2.310286000 & 4.515534000 & -0.042488000 \\ \mathrm{H} & 1.758031000 & 2.954576000 & 0.594203000 \\ \mathrm{H} & 1.627420000 & -5.096363000 & -6.613685000 \\ \mathrm{H} & 1.584332000 & -6.376298000 & -5.393337000 \\ \mathrm{H} & 0.091711000 & -5.832809000 & -6.160199000 \\ \mathrm{H} & 0.700131000 & -2.799689000 & -6.044588000 \\ \mathrm{C} & 6.057868000 & -0.338545000 & 0.505010000 \\ \mathrm{C} & 7.011655000 & 0.707693000 & 1.087074000 \\ \mathrm{H} & 6.096502000 & -1.236555000 & 1.149030000 \\ \mathrm{H} & 6.376075000 & -0.653301000 & -0.490178000 \\ \mathrm{C} & 6.506148000 & 1.209086000 & 2.440778000 \\ \mathrm{H} & 8.014370000 & 0.272175000 & 1.182156000 \\ \mathrm{H} & 7.088915000 & 1.551679000 & 0.389534000 \\ \mathrm{C} & 5.061747000 & 1.698628000 & 2.318681000 \\ \mathrm{H} & 6.548117000 & 0.386308000 & 3.169735000 \\ \mathrm{H} & 7.156846000 & 2.002667000 & 2.827160000 \\ \mathrm{C} & 4.164617000 & 0.615873000 & 1.716084000 \\ \mathrm{H} & 4.670198000 & 1.996547000 & 3.299210000 \\ \mathrm{H} & 5.032588000 & 2.585752000 & 1.672439000 \\ \mathrm{H} & 4.100789000 & -0.229886000 & 2.421049000 \\ \mathrm{H} & 3.143479000 & 0.966854000 & 1.557304000\end{array}$


Table S10. Geometry optimized coordinates for $2-\mathrm{U}(\mathrm{PN}) \mathbf{N}\left(\mathrm{CH}_{3}\right)$.

\begin{tabular}{|c|c|c|c|}
\hline & 4.357258000000 & -2.107459000000 & \\
\hline & 3.844626000000 & -1.734656000000 & -1.649437000000 \\
\hline & 104490000000 & -2.841189000000 & \\
\hline & 99000000 & .040546000000 & \\
\hline & 00 & & \\
\hline & 39000000 & & \\
\hline & 7559000000 & & \\
\hline & 1.882119000000 & 0.293386000000 & \\
\hline & 9560000000 & 000 & \\
\hline & 07000000 & 46000000 & \\
\hline & 508000000 & 25000000 & 3 \\
\hline & 075000000 & 0000 & \\
\hline & 71000000 & 00000 & 5.5 \\
\hline & 1000000 & 38000000 & 00000 \\
\hline & 25000000 & & \\
\hline & 70000000 & & \\
\hline & 50000000 & & \\
\hline & 0000000 & & \\
\hline & 11000000 & & \\
\hline & & & \\
\hline & 6.4 & & \\
\hline & 6000000 & & \\
\hline & 000 & & -1 . \\
\hline & 2.82 & 000 & 000 \\
\hline & 83000000 & & $-3.8-x-1)$ \\
\hline & 44000000 & 0000 & 000 \\
\hline & 05000000 & 00000 & 0000 \\
\hline & 24000000 & 00000 & $00000 c$ \\
\hline & 3.949319000000 & 00000 & 000000 \\
\hline & 0.468062000000 & 0000000 & 000000 \\
\hline & 0.553667000000 & 647000000 & 000000 \\
\hline$\sqrt{ }$ & 64000000 & 000000 & 0000 \\
\hline & -0.315035000000 & 87000000 & 000000 \\
\hline$\sqrt{ }$ & -1.882757000000 & 7000000 & 000000 \\
\hline$E$ & -2.962524000000 & 20000000 & 3000000 \\
\hline$C$ & -4.190277000000 & 923000000 & 3000000 \\
\hline & -4.655112000000 & 4.292806000000 & 56000000 \\
\hline & -3.491974000000 & 4.406117000000 & 3.194844000000 \\
\hline C & -2.286447000000 & 3.59 & 716 \\
\hline $\mathrm{N}$ & +24000000 & -0.23 & . \\
\hline & & 9000000 & -1.471879000000 \\
\hline & -4.421143000000 & -0.998026000000 & -0.58936700000 \\
\hline
\end{tabular}




\begin{tabular}{|c|c|c|c|}
\hline & & & \\
\hline & -6.712155000000 & .871779000000 & \\
\hline & -6.954698000000 & 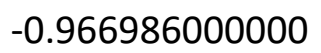 & \\
\hline & -5.630184000000 & & \\
\hline & -4.617724000000 & -0.074628000000 & \\
\hline & -0.149350000000 & & \\
\hline & 0.888871000000 & $.0320 / 2000$ & \\
\hline & 0.485752000000 & & \\
\hline & & & \\
\hline & & & \\
\hline & 30000000 & & \\
\hline & & & \\
\hline & & & \\
\hline & & & \\
\hline & & & \\
\hline & & & \\
\hline & & & \\
\hline & & & \\
\hline & & & \\
\hline & & & \\
\hline & -4.2 & 100 & -4.7 \\
\hline & 000000 & 00 & \\
\hline & 000000 & 000 & -4 \\
\hline & 00 & & 00 \\
\hline & -1.6 & & -3 \\
\hline & -1.9 & & \\
\hline & -2.1 & & \\
\hline & & & \\
\hline & & & \\
\hline & & & \\
\hline & & & \\
\hline & & & \\
\hline & & & \\
\hline & & & \\
\hline & & & \\
\hline & & & \\
\hline & & & \\
\hline & & & \\
\hline & & & \\
\hline & & & \\
\hline & & & 5.94079 \\
\hline & & & -3.22079000000 \\
\hline & & & \\
\hline & 3.466431000 & & \\
\hline
\end{tabular}




\begin{tabular}{|c|c|c|c|}
\hline & 4.822734000000 & -3.768950000000 & -3.176175000000 \\
\hline & 5.381313000000 & -5.283818000000 & -1.292912000000 \\
\hline & 3.876468000000 & -4.866707000000 & -0.478536000000 \\
\hline & 5.695728000000 & -3.558450000000 & 0.544633000000 \\
\hline & 6.203774000000 & -2.955367000000 & -1.036351000000 \\
\hline & 3.526636000000 & -2.386640000000 & 0.341147000000 \\
\hline & 4.850184000000 & -1.239030000000 & 0.117388000000 \\
\hline & 4.621636000000 & -1.037759000000 & -3.9872 \\
\hline & 4.534547000000 & 0.660530000000 & -4.4916290 \\
\hline & 2.962741000000 & -1.682591000000 & -5.674294000000 \\
\hline & 4.268826000000 & -0.784263000000 & -6.4520 \\
\hline & 1.974627000000 & -0.030326000000 & -7.2348 \\
\hline & 2.898432000000 & 1.269267000000 & $-6.4868<$ \\
\hline & 0.602784000000 & 59000000 & -5.5757 \\
\hline & 0.714249000000 & 7235000000 & -5.1173 \\
\hline & 2.390247000000 & 1.910433000000 & -3.9954 \\
\hline & 1.119896000000 & 20000000 & -3.1293 \\
\hline & -3.889651000000 & 3000000 & -0.6255 \\
\hline & -2.169028000000 & 1475000000 & -0.661867 \\
\hline & -3.476487000000 & -5.682097000000 & -1.4712 \\
\hline & 7.269584000000 & -4.676032000000 & \\
\hline & -2.423068000000 & -5.934320000000 & -3.767970000000 \\
\hline & -1.286039000000 & 49000000 & -2.553790 \\
\hline & & -3.979225000000 & -4.580003 \\
\hline & -2.747774000000 & -3.625462000000 & -4.615109000000 \\
\hline & -0.767279000000 & -2.764295000000 & -2.4373130 \\
\hline & -1.522849000000 & -1.715474000000 & -3.637285 \\
\hline & -1.754908000000 & 1.365573000000 & -3.773448 \\
\hline & -2.095109000000 & 1.668798000000 & -2.064426 \\
\hline & -3.069750000000 & 62000000 & -3.59163 \\
\hline & -4.318369000000 & 2.606924000000 & -2.656344 \\
\hline & -5.050763000000 & 2.783885000000 & -5.02912 \\
\hline & -3.549547000000 & 51000000 & $-5.56742 \varepsilon$ \\
\hline & -5.716904000000 & 0.694729000000 & -3.871759 \\
\hline & -5.314594000000 & 0.314501000000 & -5.549718000000 \\
\hline & -4.281935000000 & -1.329135000000 & -3.942169000000 \\
\hline & -3.087950000000 & -0.468250000000 & -4.910997 \\
\hline & -5.436566000000 & -1.973336000000 & -2.130104000000 \\
\hline & -6.057894000000 & -0.352000000000 & -1.774453000000 \\
\hline & -6.369838000000 & -2.857259000000 & -0.044337000000 \\
\hline & -7.641333000000 & -2.029985000000 & -0.947564000000 \\
\hline & -7.659945000000 & -1.434133000000 & 1.520060000000 \\
\hline & -7.420903000000 & -0.030013000000 & 0.483910000000 \\
\hline & -5.784397000000 & 0.071750000000 & 2.332747 \\
\hline
\end{tabular}




\begin{tabular}{|c|c|c|c|}
\hline - & -5.212922000000 & -1.558059000000 & 1.961327000000 \\
\hline & -4.987945000000 & 0.903612000000 & 0.162555000000 \\
\hline & -3.646932000000 & 0.094768000000 & 0.996781000000 \\
\hline & -3.792022000000 & 4.055274000000 & 4.189813000000 \\
\hline & -4.991495000000 & 4.469701000000 & 0.066837000000 \\
\hline & -3.225869000000 & 2.757860000000 & 0.354517000000 \\
\hline & -1.439236000000 & 3.718222000000 & 3.394816000000 \\
\hline & -2.540425000000 & 2.524038000000 & 2.701920000000 \\
\hline & -3.196442000000 & 5.458593000000 & 3.297973000000 \\
\hline & -5.489826000000 & 4.933713000000 & 2.515670000000 \\
\hline & -5.031788000000 & 3.260120000000 & 2.213497000000 \\
\hline & -3.938112000000 & 5.714507000000 & 0.744974000000 \\
\hline & -2.600474000000 & 4.113180000000 & -0.587075000000 \\
\hline & -2.506103000000 & -0.535761000000 & 5.117360000000 \\
\hline & -2.160265000000 & 1.126102000000 & 4.634472000000 \\
\hline & -2.135310000000 & 0.031855000000 & 7.492860000000 \\
\hline & -3.355629000000 & 1.105922000000 & 6.799134000000 \\
\hline & -1.643772000000 & 2.362012 & 8.194793000000 \\
\hline & -1.694673000000 & 2.9027730 & 144000000 \\
\hline & 0.664475000000 & 2.628131000000 & 2795000000 \\
\hline & 0.361915000000 & 0.983172 & 7.728518000000 \\
\hline & 0.120101000000 & 1.9767060 & 4.8388280 \\
\hline & 1.382584000000 & 0.914815000000 & 290000000 \\
\hline & 2.189334000000 & -1.685574000000 & 2.601161000000 \\
\hline & 2.384435000000 & -3.138234000000 & 3.599758000000 \\
\hline & 4.036150000000 & -0.571168000000 & 3.791120000000 \\
\hline & 4.593285000000 & -2.138718000000 & 3.194712000000 \\
\hline & 4.280684000000 & -3.152333000000 & 5.426157000000 \\
\hline & 5.304955000000 & -1.729543000000 & 5.595185000000 \\
\hline & 3.478184000000 & -1.829121000000 & 7.352054000000 \\
\hline & 3.363555000000 & -0.379904000000 & 6.348629000000 \\
\hline & 1.732325000000 & -2.948708000000 & 6.054822000000 \\
\hline & 1.126799000000 & -1.372463000000 & 6.606836000000 \\
\hline & -2.271549000000 & -2.533334000000 & 5.891053000000 \\
\hline & -0.543391000000 & -2.755368000000 & 6.145910000000 \\
\hline & -1.800753000000 & -4.905275000000 & 6.289157000000 \\
\hline & -0.617373000000 & -4.933077000000 & 4.974434000000 \\
\hline & -3.623233000000 & -4.406048000000 & 4.694413000000 \\
\hline & -2.781784000000 & -5.855355000000 & 4.152920000000 \\
\hline & -1.481555000000 & -4.532421000000 & 2.508432000000 \\
\hline & -3.199840000000 & -4.211180000000 & 2.265010000000 \\
\hline & -1.809914000000 & -2.111081000000 & 2.227795000000 \\
\hline & -3.058942000000 & -2.105384000000 & 3.485697000000 \\
\hline & 1.845517000000 & 4.534562000000 & -1.293359000000 \\
\hline
\end{tabular}




$\begin{array}{rrrr}\text { H } & 1.043729000000 & 2.955081000000 & -1.416006000000 \\ \mathrm{H} & 1.296018000000 & 4.115712000000 & -3.643159000000 \\ \mathrm{H} & -0.392006000000 & 3.795837000000 & -3.237435000000 \\ \mathrm{H} & 1.080227000000 & 6.465726000000 & -2.926173000000 \\ \mathrm{H} & -0.189494000000 & 6.119880000000 & -4.095693000000 \\ \mathrm{H} & -1.046774000000 & 7.378160000000 & -2.066800000000 \\ \mathrm{H} & -1.837863000000 & 5.811341000000 & -2.263537000000 \\ \mathrm{H} & 0.474902000000 & 6.469653000000 & -0.376726000000 \\ \mathrm{H} & -1.196355000000 & 6.123705000000 & 0.100687000000 \\ \mathrm{H} & 1.170633000000 & 6.197924000000 & 1.823788000000 \\ \mathrm{H} & -0.463703000000 & 5.894641000000 & 2.437160000000 \\ \mathrm{H} & 1.050328000000 & 6.860763000000 & 4.178853000000 \\ \mathrm{H} & 0.457015000000 & 5.262819000000 & 4.644116000000 \\ \mathrm{H} & 3.199920000000 & 5.889880000000 & 3.432450000000 \\ \mathrm{H} & 2.900336000000 & 5.388024000000 & 5.093708000000 \\ \mathrm{H} & 3.656471000000 & 3.467151000000 & 3.613607000000 \\ \mathrm{H} & 2.060324000000 & 3.185618000000 & 4.311613000000 \\ \mathrm{H} & 2.681767000000 & 4.166532000000 & 1.489214000000 \\ \mathrm{H} & 2.007578000000 & 2.585992000000 & 1.933854000000 \\ \mathrm{H} & 6.454525000000 & 0.239791000000 & -0.753517000000 \\ \mathrm{H} & 5.932263000000 & -0.351889000000 & -2.329320000000 \\ \mathrm{H} & 7.649717000000 & 1.485010000000 & -2.489386000000 \\ \mathrm{H} & 6.133083000000 & 1.930621000000 & -3.279482000000 \\ \mathrm{H} & 7.047412000000 & 2.791734000000 & -0.486252000000 \\ \mathrm{H} & 6.906266000000 & 3.837073000000 & -1.895725000000 \\ \mathrm{H} & 4.924950000000 & 4.041908000000 & -0.327267000000 \\ \mathrm{H} & 4.440911000000 & 3.494320000000 & -1.936937000000 \\ \mathrm{H} & 4.862252000000 & 1.735065000000 & 0.529177000000 \\ \mathrm{H} & 3.304945000000 & 2.091219000000 & -0.237961000000 \\ \mathrm{H} & -0.217561000000 & -3.945432000000 & 0.172078000000 \\ \mathrm{H} & 1.527750000000 & -3.773472000000 & 0.396115000000 \\ & 0.429600000000 & -3.453196000000 & 1.752031000000\end{array}$

Table S11. Geometry optimized coordinates for 2-U(PN)O.

$\begin{array}{lrrr}\text { C } & 1.746998000 & 4.400070000 & -0.185579000 \\ \text { N } & 0.374197000 & 4.756756000 & -0.508254000 \\ \text { C } & 0.248204000 & 6.037552000 & -1.187201000 \\ \text { C } & 0.852019000 & 7.170881000 & -0.354193000 \\ \text { C } & 2.303275000 & 6.852802000 & 0.013512000 \\ \text { C } & 2.400806000 & 5.478615000 & 0.680556000 \\ \text { P } & -0.904868000 & 3.620722000 & -0.299175000 \\ \text { N } & -2.122428000 & 4.277355000 & 0.747434000\end{array}$




\begin{tabular}{|c|c|c|c|}
\hline & -1.951869000 & 4.005796000 & 2.173217000 \\
\hline & -3.296345000 & 4.079613000 & 2.897010000 \\
\hline & -3.972298000 & 5.430910000 & 0010940 \\
\hline & -4.073525000 & 5.715854000 & 1.162434000 \\
\hline & -2.706473000 & 5.588968000 & 0.48709000 \\
\hline & -0.368003000 & 2.246474000 & 0.277145000 \\
\hline & -0.128296000 & 0.127535000 & -0.200612000 \\
\hline & -0.550134000 & 0.462636000 & -1.927016000 \\
\hline & 0.290749000 & -0.239027000 & 1.772157000 \\
\hline & 0.656189000 & -0.614628000 & 3.2697530 \\
\hline & -0.765687000 & -0.953216000 & 4.1797680 \\
\hline & -2.034828000 & -0.385566000 & 3.7196570 \\
\hline & -3.206453000 & -1.225455000 & 4.2268050 \\
\hline & -3.165336000 & -1.386143000 & 5.7468330 \\
\hline & 7000 & 1000 & \\
\hline & -0.6700 & 32672000 & $5.6327 \varepsilon$ \\
\hline & 1.646706000 & 0.599747000 & 3.971953 \\
\hline & 1.409889000 & 1.985394000 & 3.563576 \\
\hline C & 2.7181 & & \\
\hline & 3.38 & $2.6 \varepsilon$ & \\
\hline & 3.55875 & 1.225388000 & 5.3857020 \\
\hline & 2.2268 & 0.475466000 & 5.3072370 \\
\hline & 1.593530000 & -2.018555000 & \\
\hline & 0.94 & -3.3 & \\
\hline & 1.71 & -4.4 & 4.1 \\
\hline & 3.17793 & -4.45 & $3.6866 \mathrm{C}$ \\
\hline & & & \\
\hline & & & \\
\hline & -1.83 & -1.2 & -0.0 \\
\hline & -2.879189000 & -2.085289000 & -0.8863170 \\
\hline & -4.473752000 & -1.45 & -0.7266240 \\
\hline & & & \\
\hline & -5.86 & -0.5 & 1.07945600 \\
\hline C & -7.118012000 & -1.061862000 & 0.38579300 \\
\hline & -6.783085000 & -2.285005000 & -0.4714630 \\
\hline & -5.6372 & & -1.436210 \\
\hline & & & \\
\hline & & -0.8 & -1.5 \\
\hline & 3.584886000 & -2.538770000 & $-1.5403980 c$ \\
\hline & 2.664040000 & -3.513807000 & -0.9796860 \\
\hline & 2.345637000 & -4.620280000 & -1.9873280 \\
\hline & & & \\
\hline & & -4.208730000 & $-3.0546430 c$ \\
\hline & 4.834055000 & -3.112772000 & $-2.0130360 c$ \\
\hline IV & -2.768368000 & -2.324125000 & -2.5979930 \\
\hline & -3.075372000 & -1.169042000 & -3.4516370 \\
\hline
\end{tabular}




\begin{tabular}{|c|c|c|c|}
\hline C & -3.400460000 & -1.646870000 & -4.866923000 \\
\hline C & -2.238613000 & -2.457713000 & -5.446173000 \\
\hline C & -1.837858000 & -3.581961000 & -4.488963000 \\
\hline C & -1.580565000 & -3.037168000 & -3.083588000 \\
\hline $\mathrm{N}$ & -2.832027000 & -3.745567000 & -0.389503000 \\
\hline C & -3.702380000 & -4.755127000 & -0.982133000 \\
\hline C & -3.085441000 & -6.148755000 & -0.838778000 \\
\hline C & -2.769535000 & -6.460550000 & 0.624640000 \\
\hline C & -1.917305000 & -5.345229000 & 1.232887000 \\
\hline C & -2.574610000 & -3.980647000 & 1.028 \\
\hline $\mathrm{N}$ & 3.228700000 & -0.265943000 & -3.20 \\
\hline C & 1.979433000 & -0.383335000 & -3.957 \\
\hline C & 57000 & 0.676193000 & -5.0 \\
\hline C & 3.139320000 & 0.595375000 & -5.97 \\
\hline C & 4.42 & 0.655075000 & -5.1 \\
\hline C & 4.414499000 & -0.407125000 & -4.0 \\
\hline $\mathrm{N}$ & 4.657747000 & -0.144279000 & -1.00 \\
\hline C & 607000 & 1.317398000 & -1.0 \\
\hline C & 6.199388000 & 1.765473000 & -0.96 \\
\hline C & 6.82372 & 1.249019000 & 0.33 \\
\hline C & 6.616364000 & -0.261624000 & 0.463157000 \\
\hline C & 5.1417 & -0.623821000 & 0.2 \\
\hline $\mathrm{N}$ & -1.740212000 & 3.770420000 & 5000 \\
\hline C & & 3.126164000 & \\
\hline C & -3.842411000 & 3.749497000 & -3.07 \\
\hline C & -3.061727000 & 3.655396000 & $-4.3 \varepsilon$ \\
\hline C & -1.651268000 & 4.223570000 & -4.21 \\
\hline C & -0.946624000 & 3.566297000 & 6176000 \\
\hline $\mathrm{H}$ & -3.584209000 & 3.250819000 & -0.9 \\
\hline $\mathrm{H}$ & -2.934100000 & 2.044981000 & -2.0 \\
\hline $\mathrm{H}$ & -4.813419000 & 3.246673000 & -3.1 \\
\hline $\mathrm{H}$ & -4.045136000 & 4.803355000 & 7023000 \\
\hline $\mathrm{H}$ & -3.595308000 & 4.172754000 & -5.1 \\
\hline $\mathrm{H}$ & -2.986919000 & 2.599742000 & 35000 \\
\hline $\mathrm{H}$ & -1.059071000 & 4.065283000 & -5.1 \\
\hline $\mathrm{H}$ & -1.706081000 & 5.307287000 & -4.0436 \\
\hline $\mathrm{H}$ & -0.812980000 & 2.491050000 & -3.2144 \\
\hline $\mathrm{H}$ & 0.047558000 & 3.995812000 & -2.880618000 \\
\hline $\mathrm{H}$ & -2.806488000 & 5.717975000 & -0.593557000 \\
\hline $\mathrm{H}$ & -2.049611000 & 6.396118000 & 0.862570000 \\
\hline $\mathrm{H}$ & -4.766183000 & 5.001806000 & 0.698228000 \\
\hline $\mathrm{H}$ & -4.474945000 & 6.720862000 & 57000 \\
\hline $\mathrm{H}$ & -4.963442000 & 5.459881000 & 3.129401000 \\
\hline $\mathrm{H}$ & -3.375407000 & 6.221562000 & 50000 \\
\hline $\mathrm{H}$ & -3.142472000 & 3.905283000 & 3.969226000 \\
\hline $\mathrm{H}$ & -3.941737000 & 3.273460000 & 2.524 \\
\hline
\end{tabular}




\begin{tabular}{|c|c|c|}
\hline-1.258071000 & 4.737484000 & 2.630152000 \\
\hline-1.500769000 & 3.015950000 & 2.270685000 \\
\hline-1.349572000 & -3.845425000 & -2.386020000 \\
\hline-0.710950000 & -2.359852000 & -3.104969000 \\
\hline-0.938303000 & -4.095110000 & -4.850925000 \\
\hline-2.640078000 & -4.330540000 & -4.441944000 \\
\hline-2.499212000 & -2.861373000 & -6.431987000 \\
\hline-1.377473000 & -1.791203000 & -5.595646000 \\
\hline-3.620252000 & -0.780207000 & -5.502694000 \\
\hline-4.306805000 & -2.266197000 & -4.836877000 \\
\hline-2.221911000 & -0.474644000 & -3.47475 \\
\hline 3323000 & -0.629143000 & 18000 \\
\hline 276000 & 0.557843000 & -0.597 \\
\hline 5555000 & 0.026802000 & 0.56 \\
\hline 90000 & 259000 & \\
\hline 8990000 & -1.263378000 & 571000 \\
\hline 03000 & -1.306 & 1.12 \\
\hline 399000 & 9000 & -0.2 \\
\hline 1702000 & -3.118336000 & 0.1 \\
\hline 89000 & 000 & -1.0 \\
\hline 5132000 & -2.846310000 & -2.01 \\
\hline-5.9 & -1.2 & -2.1 \\
\hline 9000 & -4.513731000 & -2.0 \\
\hline 618000 & -4.7 & -0.4 \\
\hline 2575000 & 0382000 & 2000 \\
\hline-3.772894000 & 09000 & -1.25 \\
\hline 755000 & -7.430977000 & 0.7 \\
\hline 1337000 & -6.541383000 & 1.18 \\
\hline 317000 & 08000 & 2.305 \\
\hline-0.928130000 & -5.333580000 & 0.7572 \\
\hline 8774000 & 56000 & 1.6 \\
\hline 4838000 & 08000 & 1.3 \\
\hline 307000 & 8559000 & 3.4 \\
\hline 1.227324000 & -5.3817 & $3 . c$ \\
\hline 4000 & -3.5 & 2.2 \\
\hline 3.389288000 & -1.012798000 & 3.23 \\
\hline 2.970336000 & -2.212365000 & 2.00 \\
\hline 3.879178000 & -2.790414000 & 4.880440000 \\
\hline 3.738416000 & -5.188912000 & 4.272667000 \\
\hline 3.235481000 & -4.772855000 & 2.63 \\
\hline 1.646859000 & -4.226448000 & 5.198324000 \\
\hline-0.08 & -3.255932000 & 3.72 \\
\hline 4.999451000 & -1.706288000 & 0.338623000 \\
\hline 4.555099000 & -0.178182000 & \\
\hline 7.202680000 & -0.785164000 & -0.303083000 \\
\hline 6.967855000 & -0.616438000 & 1.44001000 \\
\hline
\end{tabular}




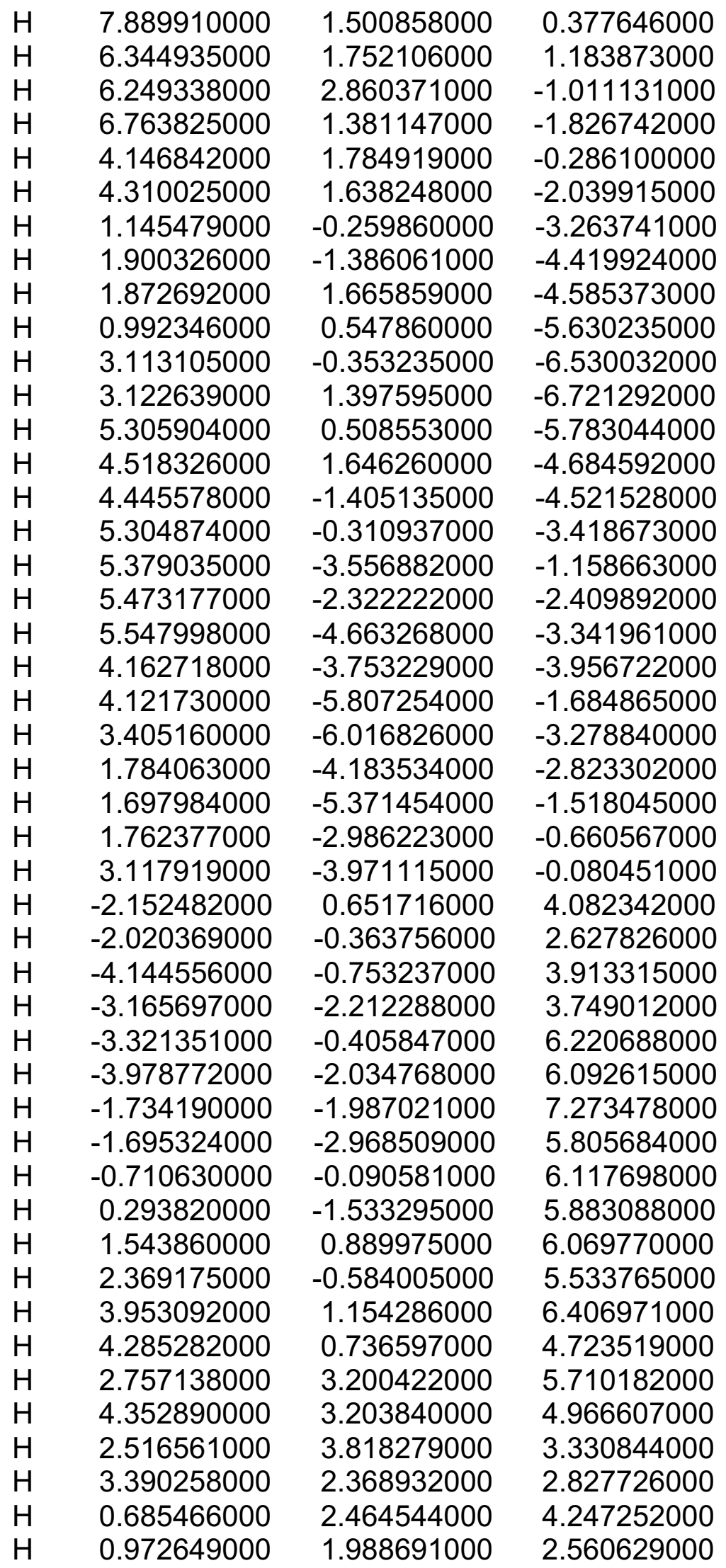




$\begin{array}{rrrr}\mathrm{H} & 0.779077000 & 5.998654000 & -2.157090000 \\ \mathrm{H} & -0.801216000 & 6.228601000 & -1.415897000 \\ \mathrm{H} & 0.791276000 & 8.111986000 & -0.915263000 \\ \mathrm{H} & 0.258151000 & 7.297160000 & 0.560562000 \\ \mathrm{H} & 2.913362000 & 6.852646000 & -0.901861000 \\ \mathrm{H} & 2.717249000 & 7.632151000 & 0.664452000 \\ \mathrm{H} & 3.448715000 & 5.215053000 & 0.870563000 \\ \mathrm{H} & 1.889478000 & 5.506987000 & 1.651740000 \\ \mathrm{H} & 2.331220000 & 4.284880000 & -1.117239000 \\ \mathrm{H} & 1.736320000 & 3.429044000 & 0.313981000\end{array}$

Table S12. Geometry optimized parameters for 2-U(PN)NMes.

\begin{tabular}{|c|c|c|c|}
\hline $\begin{array}{c}\text { Bonding Coordinate } \\
\text { In Question }\end{array}$ & Bond Length $(\AA)$ & $\begin{array}{c}\text { Bonding Angle in } \\
\text { Question }\end{array}$ & Bond Angle $\left(^{\circ}\right)$ \\
\hline U-N5_10 & 1.97 & N1_1-U-N1_5 & 94.06 \\
\hline U-N1_3 & 2.17 & N1_1-U-N5_10 & 87.93 \\
\hline U-N1_5 & 2.15 & N5_10-U-N1_5 & 111.31 \\
\hline$U-N 1 \_1$ & 2.15 & N5_10-U-N1_2 & 88.32 \\
\hline U-N1_2 & 2.15 & N1_2-U-N1_5 & 95.06 \\
\hline N5_10-C & 1.36 & N1_2-U-N1_3 & 88.95 \\
\hline N1_3-P1_3 & 1.58 & N1_3-U-N1_5 & 112.71 \\
\hline N1_5-P1_5 & 1.58 & N1_3-U-N1_1 & 87.97 \\
\hline N1_1-P1_1 & 1.59 & N1_3-U-N5_10 & 143.40 \\
\hline N1_2-P1_2 & 1.59 & N1_1-U-N1_2 & 160.80 \\
\hline
\end{tabular}

Table S13. WBI bond indices for 2-U(PN)NMes.

\begin{tabular}{|c|c|}
\hline Bonding Coordinate & WBI \\
\hline U-N5_10 & 1.9 \\
\hline$U-N 1 \_3$ & 1.1 \\
\hline$U-N 1 \_5$ & 1.1 \\
\hline$U-N 1 \_1$ & 1.2 \\
\hline$U-N 1 \_2$ & 1.2 \\
\hline
\end{tabular}




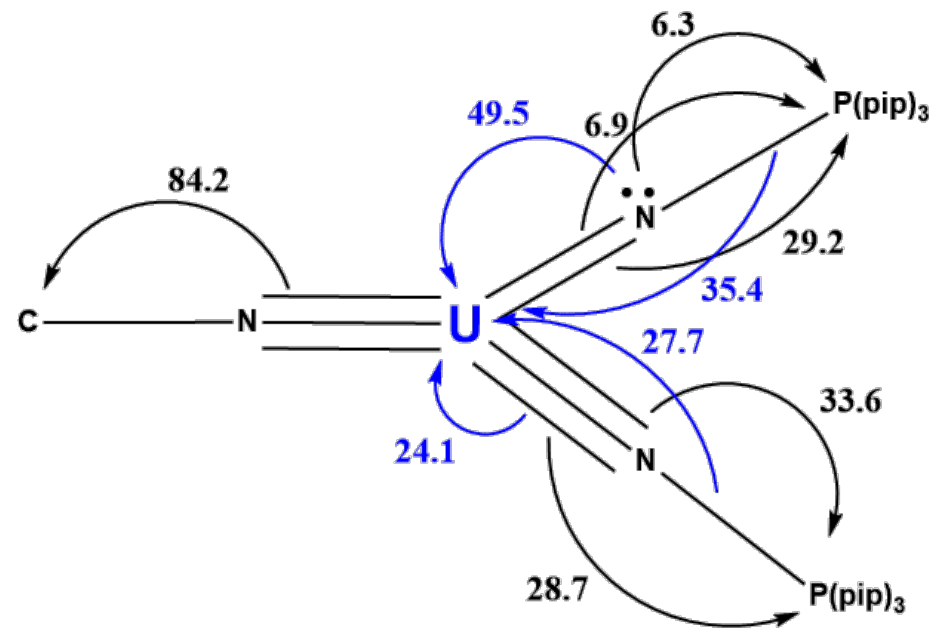

Figure S26. A depiction of the energy movement within the equatorial plane of the complex. In blue is the donations of energy to the $U$ atom. The values are in $\mathrm{Kcal} / \mathrm{mol}$ and are based on the overall donations.

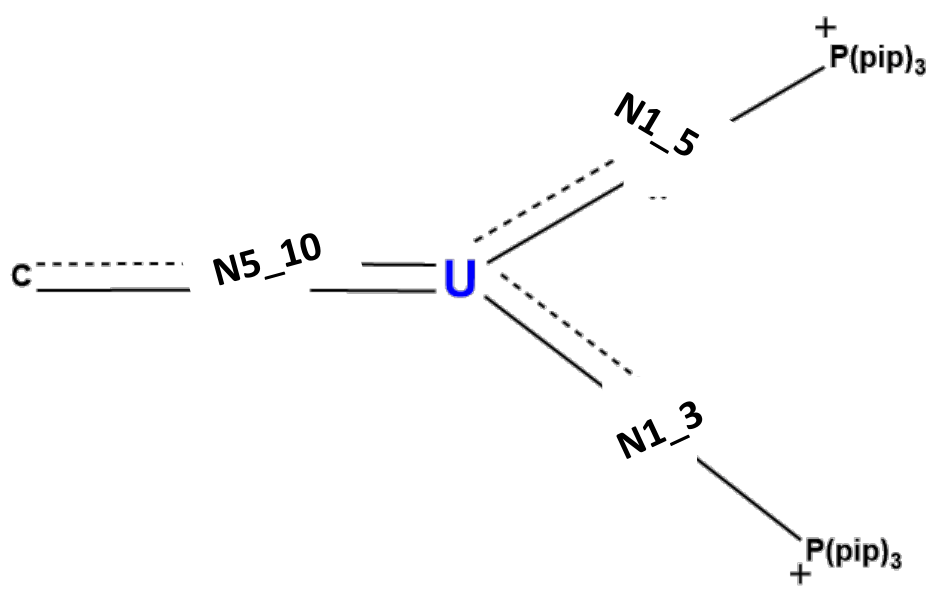

Figure S27. Bonding seen within the equatorial plane of the complex. Note that the 'Ione pair' on the nitrogen 30 and 8 are considered to mostly be delocalized along the $\mathrm{U}-\mathrm{N}$ 

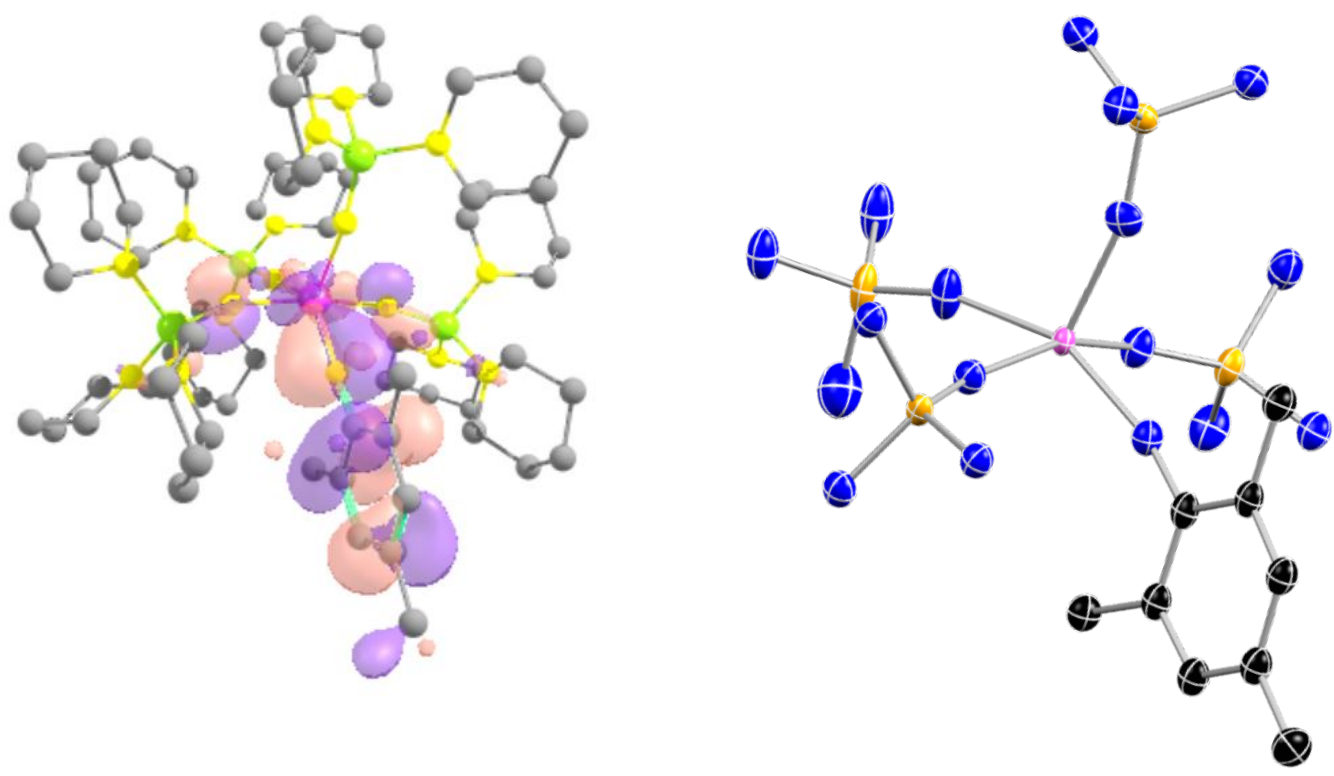

Figure S28. HOMO of 2-U(PN)NMes: highlighting one of the Pi interactions in the $\mathrm{U}_{-}$ $\mathrm{N}_{\text {MES }}$ coordinate. To be clear of the perspective a stick and ball structure is also displayed. The Hydrogen atoms have been omitted for clarity.

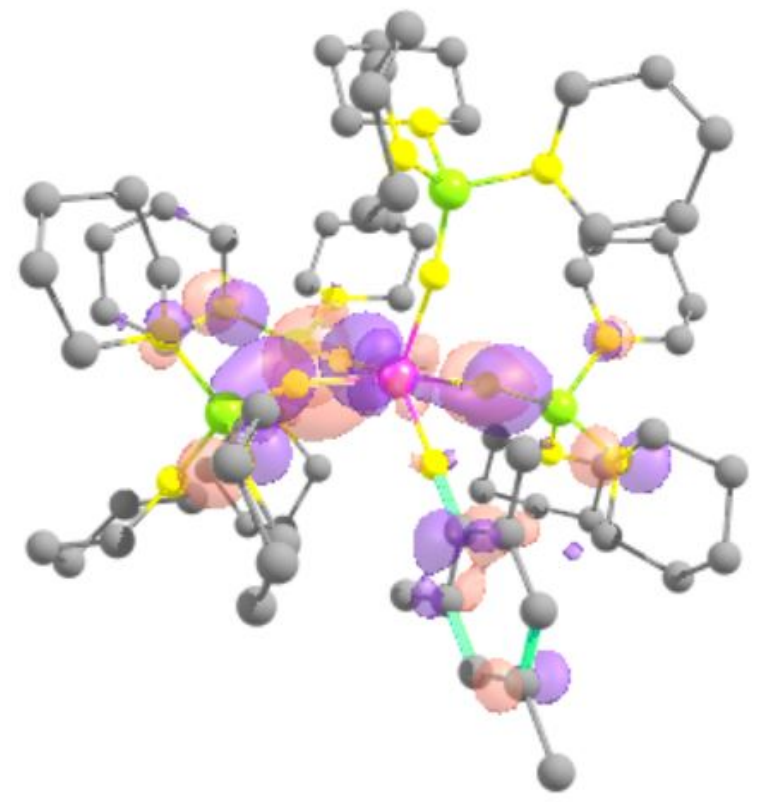

Figure S29. HOMO-1 of 2-U(PN)NMes: highlighting the U-N-P coordinate. Note the clear delocalization of the electrons across the plane. The Hydrogen atoms have been omitted for clarity. 


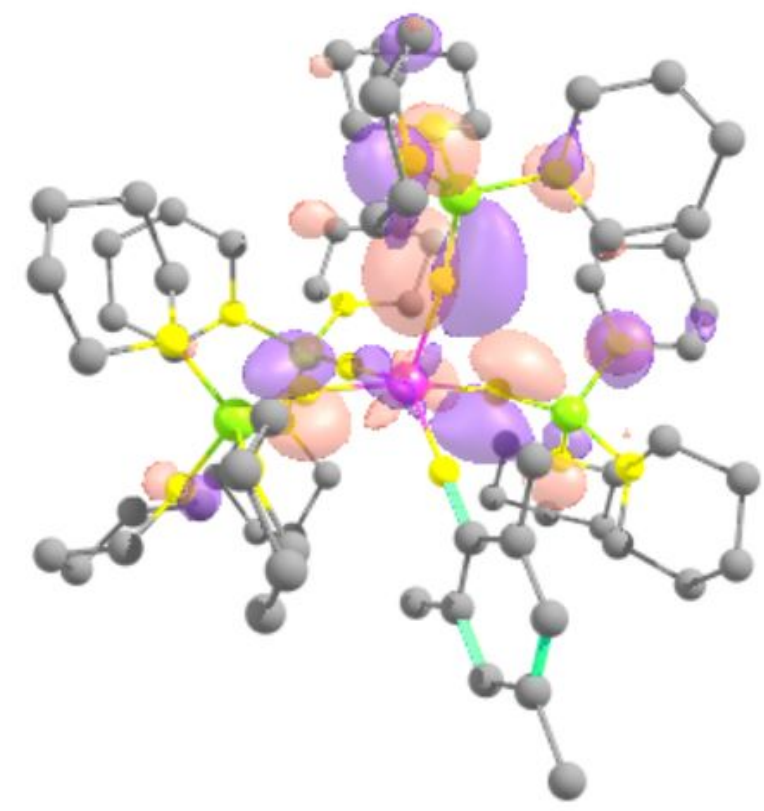

Figure S30. HOMO-2 of 2-U(PN)NMes: highlighting the second $\mathrm{P}$ interaction of the $\mathrm{U}-$ $\mathrm{N}_{\mathrm{MES}}$ bond. (Left side on the complex) The Hydrogen atoms have been omitted for clarity.

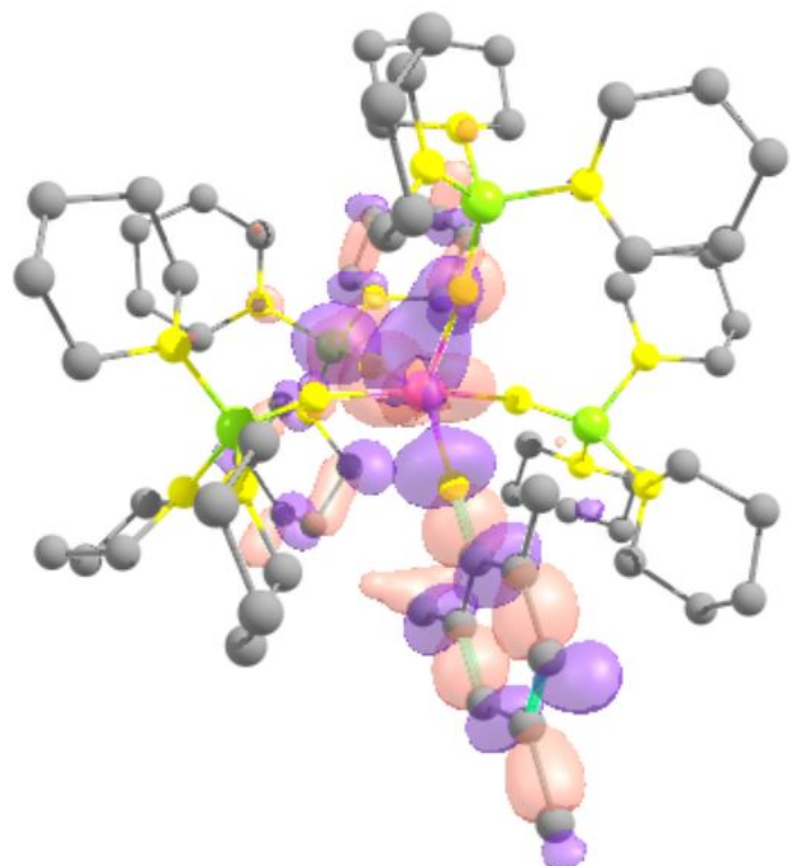

Figure S31. A lower energy MO of 2-U(PN)NMes: highlighting the sigma interaction of the U-N $\mathrm{N}_{\text {MES }}$ bond. (Left side on the complex) The Hydrogen atoms have been omitted for clarity. 


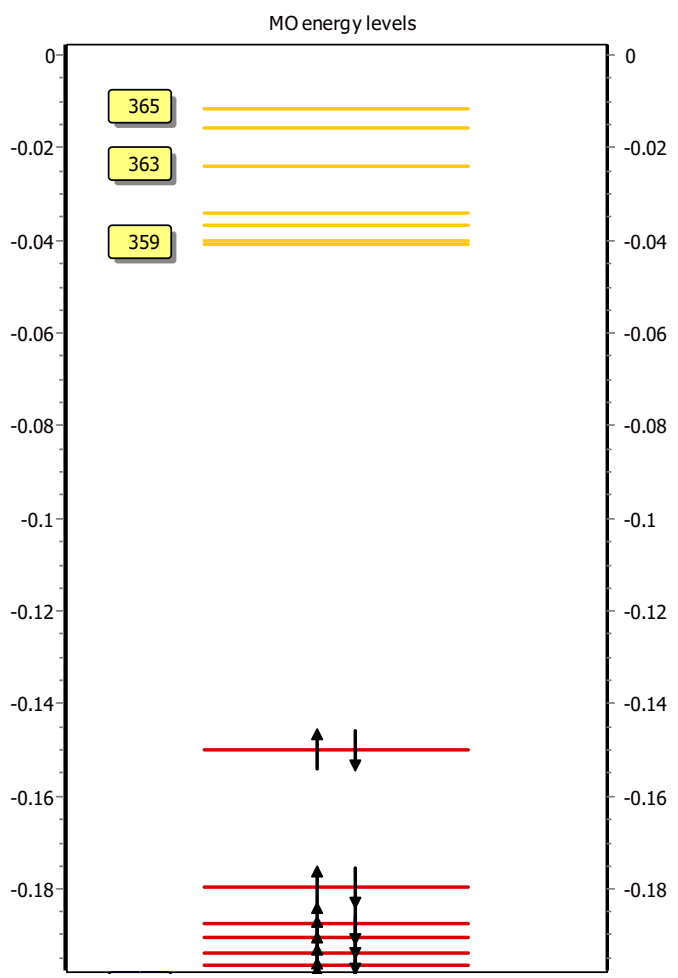

Figure S32. Frontier orbital energy diagram for 2-U(PN)NMes.

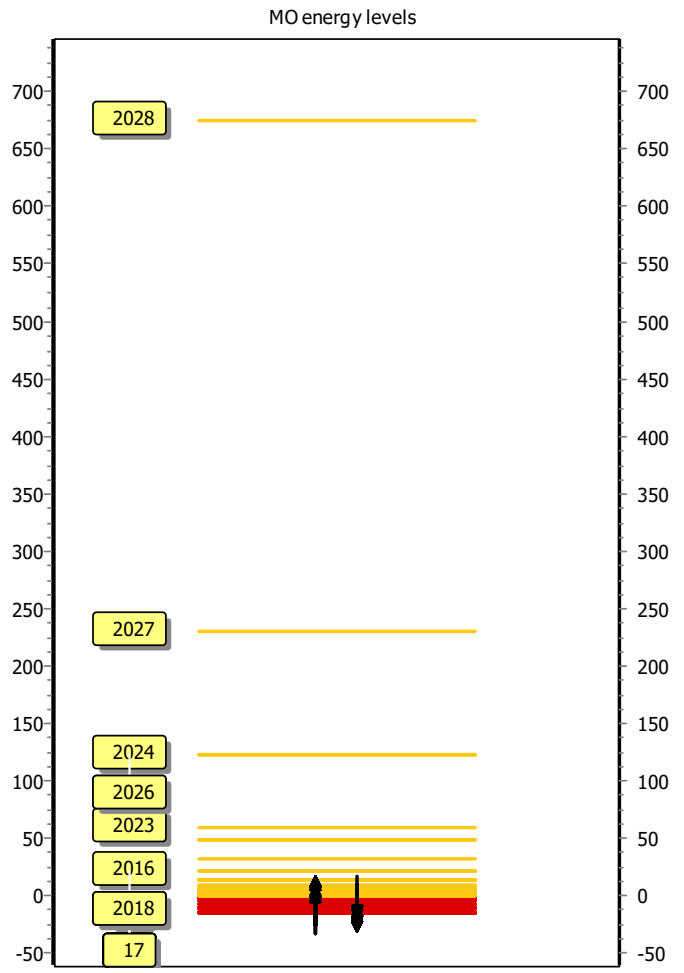

Figure S33. Full energy diagram for 2-U(PN)NMes. 
E (a.u)
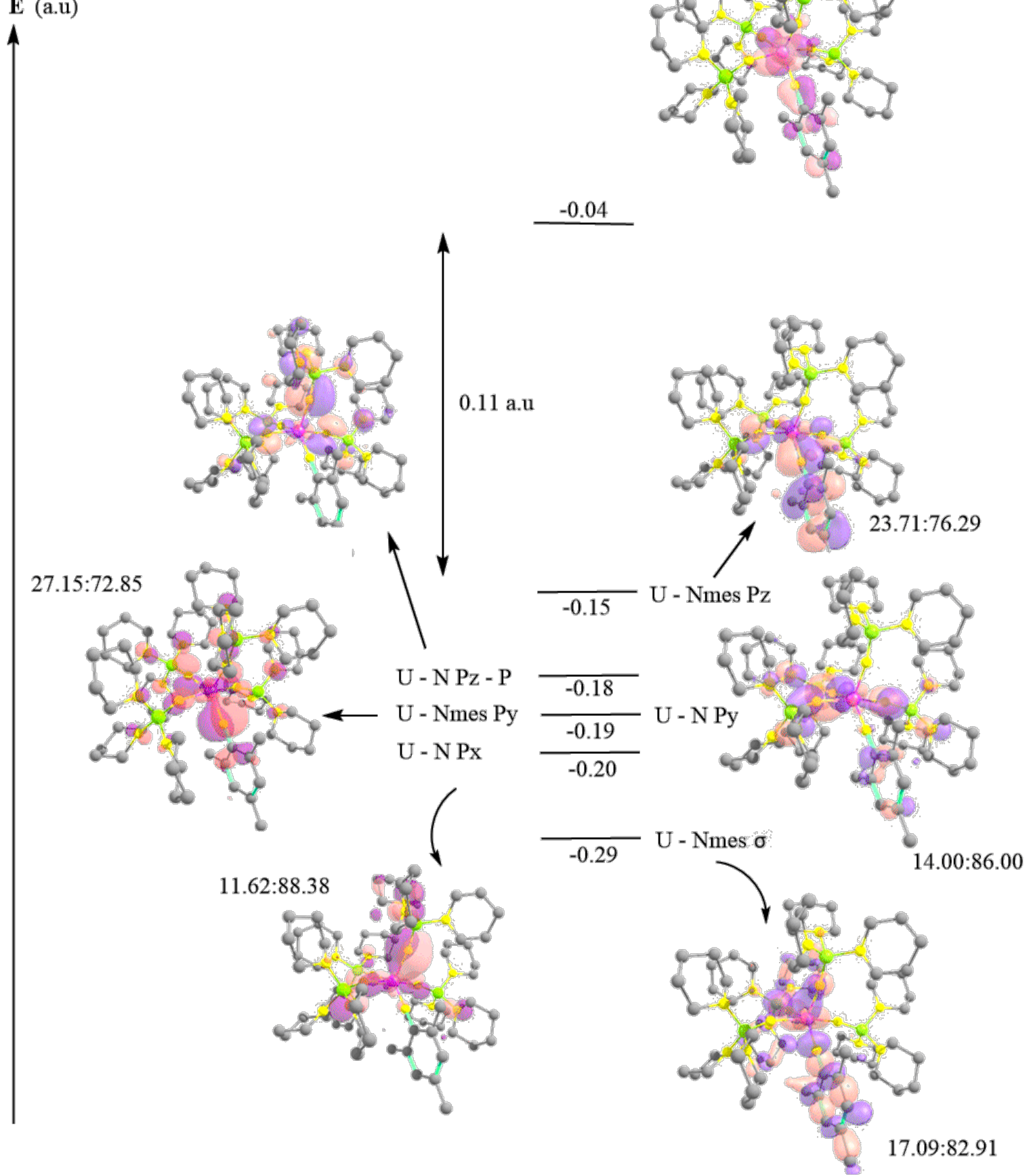

Figure S34. A diagram of the molecular orbitals for 2-U(PN)NMes, indicating the strong interactions between the various HOMOs and $U-N_{\text {MES }}$ bond. Additionally, the equatorial ligands and apical $\mathrm{P}(\mathrm{pip})_{3}$ interactions. Note the LUMO takes no part in the interactions of the ligands - it is to high in energy. 
Table S14. Contributions to U-N1_3 bond in 2-U(PN)NMes.

\begin{tabular}{|c|c|c|c|c|c|c|c|c|c|}
\hline & \multicolumn{4}{|c|}{ U } & \multicolumn{4}{|c|}{ N1_3 } & \\
\hline \multirow[t]{3}{*}{$\begin{array}{l}1^{\text {st }} \\
\text { Bond }\end{array}$} & \multicolumn{4}{|c|}{$13.16 \%$} & \multicolumn{4}{|c|}{$86.84 \%$} & $\begin{array}{l}U-N-P \\
\text { Coordinate }\end{array}$ \\
\hline & $\mathrm{s}$ & $p$ & d & $f$ & $\mathrm{~s}$ & $p$ & d & $f$ & \\
\hline & $5.72 \%$ & $19.80 \%$ & $40.89 \%$ & $33.47 \%$ & $61.64 \%$ & $38.36 \%$ & $0.00 \%$ & $0.00 \%$ & \\
\hline \multirow[t]{3}{*}{$\begin{array}{l}2^{\text {nd }} \\
\text { Bond }\end{array}$} & \multicolumn{4}{|c|}{$13.25 \%$} & \multicolumn{4}{|c|}{$86.75 \%$} & $\begin{array}{l}U-N-P \\
\text { Coordinate }\end{array}$ \\
\hline & $\mathrm{s}$ & $p$ & d & $\mathrm{f}$ & $\mathrm{s}$ & $\mathrm{p}$ & d & $\mathrm{f}$ & \\
\hline & $0.10 \%$ & $11.05 \%$ & $39.78 \%$ & $49.04 \%$ & $0.02 \%$ & $99.97 \%$ & $0.01 \%$ & $0.00 \%$ & \\
\hline $\begin{array}{l}3^{\text {rd }} \\
\text { Bond }\end{array}$ & \multicolumn{4}{|c|}{$10.02 \%$} & \multicolumn{4}{|c|}{$89.98 \%$} & $\begin{array}{l}\mathrm{U}-\mathrm{N}-\mathrm{P} \\
\text { Coordinate }\end{array}$ \\
\hline & $\mathrm{s}$ & $p$ & d & $f$ & $\mathrm{~s}$ & $p$ & d & $f$ & \\
\hline & $0.07 \%$ & $16.80 \%$ & $13.22 \%$ & $69.81 \%$ & $0.07 \%$ & $99.92 \%$ & $0.01 \%$ & $0.00 \%$ & \\
\hline
\end{tabular}

Table S15. Contributions to U-N1_2 bond in 2-U(PN)NMes.

\begin{tabular}{|c|c|c|c|c|c|c|c|c|c|}
\hline & \multicolumn{4}{|c|}{ U } & \multicolumn{4}{|c|}{ N1_2 } & \\
\hline $\begin{array}{c}1^{\text {st }} \\
\text { Bond }\end{array}$ & \multicolumn{4}{|c|}{$13.37 \%$} & \multicolumn{4}{|c|}{$86.63 \%$} & $\begin{array}{l}\mathrm{U}-\mathrm{N}-\mathrm{P} \\
\text { Coordinate }\end{array}$ \\
\hline & $\mathrm{s}$ & $p$ & d & $f$ & $s$ & $p$ & $d$ & $f$ & \\
\hline & $4.93 \%$ & $20.86 \%$ & $43.00 \%$ & $31.12 \%$ & $61.31 \%$ & $38.69 \%$ & $0.00 \%$ & $0.00 \%$ & \\
\hline $\begin{array}{l}2^{\text {nd }} \\
\text { Bond }\end{array}$ & \multicolumn{4}{|c|}{$14.09 \%$} & \multicolumn{4}{|c|}{$85.91 \%$} & $\begin{array}{l}\mathrm{U}-\mathrm{N}-\mathrm{P} \\
\text { Coordinate }\end{array}$ \\
\hline & $s$ & $p$ & $d$ & $f$ & $\mathrm{~s}$ & $P$ & $d$ & $f$ & \\
\hline & $0.17 \%$ & $4.88 \%$ & $39.31 \%$ & $55.62 \%$ & $0.09 \%$ & $99.90 \%$ & $0.01 \%$ & $0.00 \%$ & \\
\hline $\begin{array}{l}3^{\text {rd }} \\
\text { Bond }\end{array}$ & \multicolumn{4}{|c|}{$11.10 \%$} & \multicolumn{4}{|c|}{$88.90 \%$} & $\begin{array}{l}\mathrm{U}-\mathrm{N}-\mathrm{P} \\
\text { Coordinate }\end{array}$ \\
\hline & $\mathrm{s}$ & $p$ & d & $f$ & $S$ & $p$ & $d$ & $f$ & \\
\hline
\end{tabular}




\begin{tabular}{|l|l|l|l|l|l|l|l|}
\hline $0.07 \%$ & $12.39 \%$ & $37.38 \%$ & $50.08 \%$ & $0.31 \%$ & $99.68 \%$ & $0.01 \%$ & $0.00 \%$ \\
\hline
\end{tabular}

Table S16. Contributions to U-N1_5 bond in 2-U(PN)NMes.

\begin{tabular}{|c|c|c|c|c|c|c|c|c|c|}
\hline & \multicolumn{4}{|c|}{ U } & \multicolumn{4}{|c|}{ N1_5 } & \\
\hline $\begin{array}{l}1^{\text {st }} \\
\text { Bond }\end{array}$ & \multicolumn{4}{|c|}{$11.62 \%$} & \multicolumn{4}{|c|}{$88.38 \%$} & $\begin{array}{c}U-N \boldsymbol{P x} \\
\text { Interaction }\end{array}$ \\
\hline & $\mathrm{s}$ & $p$ & d & $\mathrm{f}$ & $\mathrm{s}$ & $\mathrm{p}$ & d & $\mathrm{f}$ & \\
\hline & $0.21 \%$ & $25.85 \%$ & $22.25 \%$ & $51.61 \%$ & $0.31 \%$ & $99.68 \%$ & $0.01 \%$ & $0.00 \%$ & \\
\hline $\begin{array}{l}2^{\text {nd }} \\
\text { Bond }\end{array}$ & \multicolumn{4}{|c|}{$11.78 \%$} & \multicolumn{4}{|c|}{$88.22 \%$} & $\begin{array}{l}\text { U - N Py } \\
\text { Interaction }\end{array}$ \\
\hline & $\mathrm{s}$ & $\mathrm{p}$ & d & $f$ & $\mathrm{~s}$ & $p$ & d & $f$ & \\
\hline & $0.16 \%$ & $28.53 \%$ & $27.21 \%$ & $44.01 \%$ & $0.36 \%$ & $99.63 \%$ & $0.01 \%$ & $0.00 \%$ & \\
\hline
\end{tabular}

Table S17. Contributions to U-N1_1 bond in 2-U(PN)NMes.

\begin{tabular}{|c|c|c|c|c|c|c|c|c|c|}
\hline & \multicolumn{4}{|c|}{$U$} & \multicolumn{4}{|c|}{ N1_1 } & \\
\hline $\begin{array}{c}1^{\text {st }} \\
\text { Bond }\end{array}$ & \multicolumn{4}{|c|}{$13.58 \%$} & \multicolumn{4}{|c|}{$86.42 \%$} & $\begin{array}{c}U-N P y \\
\text { Interaction }\end{array}$ \\
\hline & $\mathrm{s}$ & $\mathrm{p}$ & $d$ & $f$ & $s$ & $\mathrm{p}$ & d & $f$ & \\
\hline & $4.54 \%$ & $19.74 \%$ & $44.25 \%$ & $31.39 \%$ & $61.36 \%$ & $38.64 \%$ & 0 & 0 & \\
\hline $\begin{array}{l}2^{\text {nd }} \\
\text { Bond } \\
\text { Order }\end{array}$ & \multicolumn{4}{|c|}{$14.74 \%$} & \multicolumn{4}{|c|}{$85.26 \%$} & $\begin{array}{l}\mathrm{U}-\mathrm{N}-\mathrm{P} \\
\text { Coordinate }\end{array}$ \\
\hline & $\mathrm{s}$ & $\mathrm{p}$ & d & f & S & $\mathrm{p}$ & d & $f$ & \\
\hline & $0.05 \%$ & $3.40 \%$ & $37.28 \%$ & $59.24 \%$ & $0.01 \%$ & $99.98 \%$ & $0.01 \%$ & $0.00 \%$ & \\
\hline $\begin{array}{l}3^{\text {rd }} \\
\text { Bond }\end{array}$ & \multicolumn{4}{|c|}{$10.75 \%$} & \multicolumn{4}{|c|}{$89.25 \%$} & $\begin{array}{l}\mathrm{U}-\mathrm{N}-\mathrm{P} \\
\text { Coordinate }\end{array}$ \\
\hline & $\mathrm{s}$ & $\mathrm{p}$ & d & $f$ & $\mathrm{~s}$ & $\mathrm{P}$ & d & $f$ & \\
\hline & $0.03 \%$ & $13.64 \%$ & $37.24 \%$ & $49.00 \%$ & $0.50 \%$ & $99.48 \%$ & $0.02 \%$ & $0.00 \%$ & \\
\hline
\end{tabular}


Table S18. Contributions to U-N5_10 bond in 2-U(PN)NMes.

\begin{tabular}{|c|c|c|c|c|c|c|c|c|c|}
\hline & \multicolumn{4}{|c|}{ U } & \multicolumn{4}{|c|}{ N5_10 } & \\
\hline \multirow[t]{3}{*}{$\begin{array}{c}1^{\text {st }} \\
\text { Bond }\end{array}$} & \multicolumn{4}{|c|}{$17.09 \%$} & \multicolumn{4}{|c|}{$82.91 \%$} & $\begin{array}{c}U-N_{M E S} \\
\boldsymbol{\sigma} \\
\text { interaction }\end{array}$ \\
\hline & $\mathrm{s}$ & $p$ & d & f & $\mathrm{s}$ & $p$ & d & f & \\
\hline & $3.22 \%$ & $14.61 \%$ & $48.00 \%$ & $34.04 \%$ & $57.29 \%$ & $42.71 \%$ & $0.00 \%$ & $0.00 \%$ & \\
\hline $\begin{array}{c}2^{\text {nd }} \\
\text { Bond }\end{array}$ & \multicolumn{4}{|c|}{$27.15 \%$} & \multicolumn{4}{|c|}{$72.85 \%$} & $\begin{array}{c}U-N_{\text {MES }} \\
P y \\
\text { Interaction }\end{array}$ \\
\hline & $\mathrm{s}$ & $p$ & $d$ & $f$ & $S$ & $p$ & $d$ & $f$ & \\
\hline & $0.13 \%$ & $4.32 \%$ & $38.24 \%$ & $57.28 \%$ & $0.00 \%$ & $100.00 \%$ & $0.00 \%$ & $0.00 \%$ & \\
\hline $\begin{array}{c}3^{\text {rd }} \\
\text { Bond }\end{array}$ & \multicolumn{4}{|c|}{$23.71 \%$} & \multicolumn{4}{|c|}{$76.29 \%$} & $\begin{array}{c}U-N_{M E S} \\
\mathbf{P z} \\
\text { Interaction }\end{array}$ \\
\hline & $\mathrm{s}$ & $p$ & d & f & $\mathrm{s}$ & $p$ & d & f & \\
\hline & $0.01 \%$ & $12.29 \%$ & $19.10 \%$ & $68.54 \%$ & $0.00 \%$ & $100.00 \%$ & $0.00 \%$ & $0.00 \%$ & \\
\hline
\end{tabular}

Table S19. Natural Population Analysis (NPA) for 2-U(PN)NMes.

\begin{tabular}{|c|c|}
\hline Atom & Natural Charge \\
\hline N5_10 & -0.6 \\
\hline C(Mes) & 0.2 \\
\hline N1_1 & -1.3 \\
\hline P1_1 & 2.5 \\
\hline N1_5 & -1.3 \\
\hline P1_5 & 2.5 \\
\hline N1_3 & -1.3 \\
\hline P1_3 & 2.4 \\
\hline N1_2 & -1.3 \\
\hline P1_2 & 2.5 \\
\hline U1 & 1.3 \\
\hline
\end{tabular}

Table 20. Mulliken Charges for 2-U(PN)NMes.

\begin{tabular}{|c|c|}
\hline Atom & Mulliken Charge \\
\hline N5_10 & -0.6 \\
\hline C(Mes) & 0.1 \\
\hline N1_1 & -0.6 \\
\hline P1_1 & 0.6 \\
\hline N1_5 & -0.6 \\
\hline
\end{tabular}




\begin{tabular}{|c|c|}
\hline P1_5 & 0.6 \\
\hline N1_3 & -0.6 \\
\hline P1_3 & 0.6 \\
\hline N1_2 & -0.6 \\
\hline P1_2 & 0.6 \\
\hline U & 1.5 \\
\hline
\end{tabular}

Table 21. Mulliken Orbital occupation for 2-U(PN)NMes.

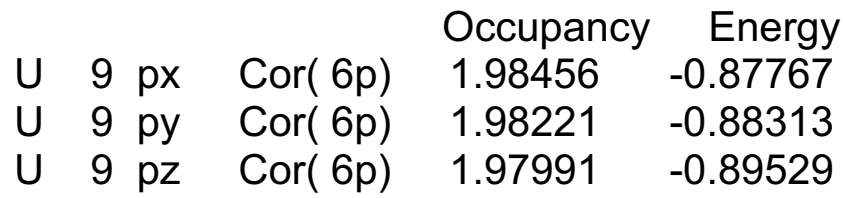

The above NBO analysis was done using the optimized geometry. According to experimental data, an assumption was made into the fact that this structure is in fact a square planar whereby, the $\mathrm{N}_{\mathrm{MES}}$ group lies on an equatorial plane trans to another ligand. With regards to that hypothesis, analysis of both a true square planar and one that is distorted - by calculation - can be compared.

Table S22. WBI of forced square pyramid

\begin{tabular}{|c|c|c|}
\hline $\begin{array}{c}\text { Bonding Coordinate } \\
\text { In Question }\end{array}$ & $\begin{array}{c}\text { WBI once the } \\
\text { system is allowed } \\
\text { to optimize }\end{array}$ & $\begin{array}{c}\text { WBI on the } \\
\text { forced square } \\
\text { planar system }\end{array}$ \\
\hline $\mathrm{U}-\mathrm{N}(\mathrm{N} 5$ 10) & 1.9 & 1.9 \\
\hline $\mathrm{U}-\mathrm{N}\left(\mathrm{N} 1 \_3\right)$ & 1.1 & 1.2 \\
\hline $\mathrm{U}-\mathrm{N}(\mathrm{N} 1$ 5 $)$ & 1.1 & 1.2 \\
\hline $\mathrm{U}-\mathrm{N}(\mathrm{N} 1$ 1) & 1.2 & 1.2 \\
\hline $\mathrm{U}-\mathrm{N}(\mathrm{N} 1$ 2) & 1.2 & 1.2 \\
\hline
\end{tabular}

Table S23. Contributions to U-N1_3 bond in forced square pyramid.

\begin{tabular}{|c|c|c|c|c|c|c|c|c|c|}
\hline & \multicolumn{4}{|c|}{ U } & \multicolumn{4}{|c|}{ N1 3} & \\
\hline $\begin{array}{l}1^{\text {st }} \\
\text { Bond }\end{array}$ & \multicolumn{4}{|c|}{$12.88 \%$} & \multicolumn{4}{|c|}{$87.12 \%$} & $\begin{array}{l}U-N-P \\
\text { Coordinate }\end{array}$ \\
\hline & $\mathrm{s}$ & $p$ & d & f & $\mathrm{s}$ & $p$ & d & f & \\
\hline & $2.72 \%$ & $7.18 \%$ & $44.58 \%$ & $45.47 \%$ & $0.56 \%$ & $99.43 \%$ & $0.01 \%$ & $0.00 \%$ & \\
\hline
\end{tabular}


Table S24. Contributions to U-N1_2 bond in forced square pyramid.

\begin{tabular}{|c|c|c|c|c|c|c|c|c|c|}
\hline & \multicolumn{5}{|c|}{ U } & \multicolumn{5}{c|}{ N1_2 } & \\
\hline $\begin{array}{c}1^{\text {1t }} \\
\text { Bond }\end{array}$ & \multicolumn{5}{|c|}{$14.67 \%$} & \multicolumn{5}{c|}{$85.33 \%$} & & $\begin{array}{c}\text { U-N - P } \\
\text { Coordinate }\end{array}$ \\
\hline & $\mathrm{s}$ & $\mathrm{p}$ & $\mathrm{d}$ & $\mathrm{f}$ & $\mathrm{s}$ & $\mathrm{p}$ & $\mathrm{d}$ & $\mathrm{f}$ & \\
\hline & $2.28 \%$ & $4.83 \%$ & $35.50 \%$ & $57.36 \%$ & $1.73 \%$ & $98.26 \%$ & $0.01 \%$ & $0.00 \%$ & \\
\hline & & & & & & & & & \\
\hline $\begin{array}{c}2^{\text {nd }} \\
\text { Bond }\end{array}$ & \multicolumn{7}{|c|}{$12.36 \%$} & & \multicolumn{3}{|c|}{$87.64 \%$} & & $\begin{array}{c}\text { U-N - P } \\
\text { Coordinate }\end{array}$ \\
\hline & $\mathrm{s}$ & $\mathrm{p}$ & $\mathrm{d}$ & $\mathrm{f}$ & $\mathrm{s}$ & $\mathrm{P}$ & $\mathrm{d}$ & $\mathrm{f}$ & \\
\hline & $0.68 \%$ & $4.04 \%$ & $37.63 \%$ & $57.62 \%$ & $0.00 \%$ & $99.99 \%$ & $0.01 \%$ & $0.00 \%$ & \\
\hline & & & & & & & & & \\
\hline
\end{tabular}

Moreover, the $\mathrm{U}-\mathrm{N} 1$ 2 2 corrdinate only reports two bonds in comparison to three; seen in both optimal and experimental structures. Whilst only two bonds are reported in the square planar system the $1^{\text {st }}$ bond is entirely a p interaction from the N1_2 atom. This is in contrast to a strong sigma interaction seen in the other optimal and experimental structures.

Table S25. Contributions to U-N1_5 bond in forced square pyramid.

\begin{tabular}{|c|c|c|c|c|c|c|c|c|c|}
\hline & \multicolumn{4}{|c|}{ U } & \multicolumn{4}{|c|}{ N1_5 } & \\
\hline $\begin{array}{l}1^{1 \text { st }} \\
\text { Bond }\end{array}$ & \multicolumn{4}{|c|}{$14.62 \%$} & \multicolumn{4}{|c|}{$85.38 \%$} & $\begin{array}{c}U-N \boldsymbol{P x} \\
\text { Interaction }\end{array}$ \\
\hline & $\mathrm{s}$ & $p$ & d & $f$ & $\mathrm{~s}$ & $p$ & d & $f$ & \\
\hline & $22.63 \%$ & $8.54 \%$ & $31.86 \%$ & $36.91 \%$ & $10.39 \%$ & $89.60 \%$ & $0.01 \%$ & $0.00 \%$ & \\
\hline $\begin{array}{l}2^{\text {nd }} \\
\text { Bond }\end{array}$ & \multicolumn{4}{|c|}{$14.04 \%$} & \multicolumn{4}{|c|}{$85.96 \%$} & $\begin{array}{c}\mathrm{U}-\mathrm{N} \text { Py } \\
\text { Interaction }\end{array}$ \\
\hline & $\mathrm{s}$ & $p$ & $\mathrm{~d}$ & $\mathrm{f}$ & $\mathrm{s}$ & $p$ & $\mathrm{~d}$ & $f$ & \\
\hline & $0.11 \%$ & $2.54 \%$ & $29.53 \%$ & $67.80 \%$ & $0.91 \%$ & $99.79 \%$ & $0.02 \%$ & $0.00 \%$ & \\
\hline
\end{tabular}

The U-N1_5 bond is similar to both structures analysed previously. 
Table S26. Contributions to U-N1_1 bond in forced square pyramid.

\begin{tabular}{|c|c|c|c|c|c|c|c|c|c|}
\hline & \multicolumn{4}{|c|}{ U } & \multicolumn{4}{|c|}{ N1_1 } & \\
\hline $\begin{array}{c}1^{\text {st }} \\
\text { Bond }\end{array}$ & \multicolumn{4}{|c|}{$16.38 \%$} & \multicolumn{4}{|c|}{$83.62 \%$} & $\begin{array}{c}U-N P y \\
\text { Interaction }\end{array}$ \\
\hline & $\mathrm{s}$ & $p$ & d & $f$ & $\mathrm{~s}$ & $p$ & d & $f$ & \\
\hline & $6.75 \%$ & $5.01 \%$ & $30.74 \%$ & $57.47 \%$ & $7.32 \%$ & $92.67 \%$ & $0.01 \%$ & $0.00 \%$ & \\
\hline \multirow[t]{3}{*}{$\begin{array}{l}2^{\text {nd }} \\
\text { Bond } \\
\text { Order }\end{array}$} & \multicolumn{4}{|c|}{$12.51 \%$} & \multicolumn{4}{|c|}{$87.49 \%$} & $\begin{array}{l}\mathrm{U}-\mathrm{N}-\mathrm{P} \\
\text { Coordinate }\end{array}$ \\
\hline & $s$ & $p$ & d & f & $\mathrm{s}$ & $p$ & d & $\mathrm{f}$ & \\
\hline & $1.76 \%$ & $3.48 \%$ & $41.99 \%$ & $52.73 \%$ & $0.53 \%$ & $99.46 \%$ & $0.01 \%$ & $0.00^{\circ} \%$ & \\
\hline
\end{tabular}

The U-N1_1 bond coordinate follows a similar trend to the optimized sturcture - but only taking into account 2 bonds relative to the three bonds seen in the optimised structure - another $\pi$ inteaction.

Table S27. Contributions to U-N5_10 bond in forced square pyramid.

\begin{tabular}{|c|c|c|c|c|c|c|c|c|c|}
\hline & \multicolumn{4}{|c|}{$U$} & \multicolumn{4}{|c|}{ N5_10 } & \\
\hline \multirow{3}{*}{$\begin{array}{c}1^{\text {st }} \\
\text { Bond }\end{array}$} & \multicolumn{4}{|c|}{$19.72 \%$} & \multicolumn{4}{|c|}{$80.28 \%$} & $U-N_{M E S}$ \\
\hline & $s$ & $\mathrm{p}$ & $d$ & $f$ & s & $\mathrm{p}$ & $d$ & $f$ & \\
\hline & $17.35 \%$ & $9.39 \%$ & $33.11 \%$ & $40.07 \%$ & $56.45 \%$ & $43.55 \%$ & $0.00 \%$ & $0.00 \%$ & \\
\hline $2^{\text {nd }}$ & \multicolumn{4}{|c|}{$27.32 \%$} & \multicolumn{4}{|c|}{$72.68 \%$} & $U-N_{M E S}$ \\
\hline \multirow{3}{*}{$\begin{array}{l}3^{\text {rd }} \\
\text { Bond }\end{array}$} & s & $p$ & $\mathrm{~d}$ & $f$ & $s$ & $p$ & d & $\mathrm{f}$ & \\
\hline & $0.18 \%$ & $1.55 \%$ & $28.71 \%$ & $69.53 \%$ & $0.00 \%$ & $100.00 \%$ & $0.00 \%$ & $0.00 \%$ & \\
\hline & \multicolumn{4}{|c|}{$27.16 \%$} & \multicolumn{4}{|c|}{$72.84 \%$} & $U-N_{M E S}$ \\
\hline & $\mathrm{s}$ & $p$ & $d$ & $f$ & $\mathrm{~s}$ & $p$ & d & $f$ & \\
\hline & $0.03 \%$ & $1.13 \%$ & $43.35 \%$ & $55.46 \%$ & $0.01 \%$ & $99.99 \%$ & $0.00 \%$ & $0.00 \%$ & \\
\hline
\end{tabular}



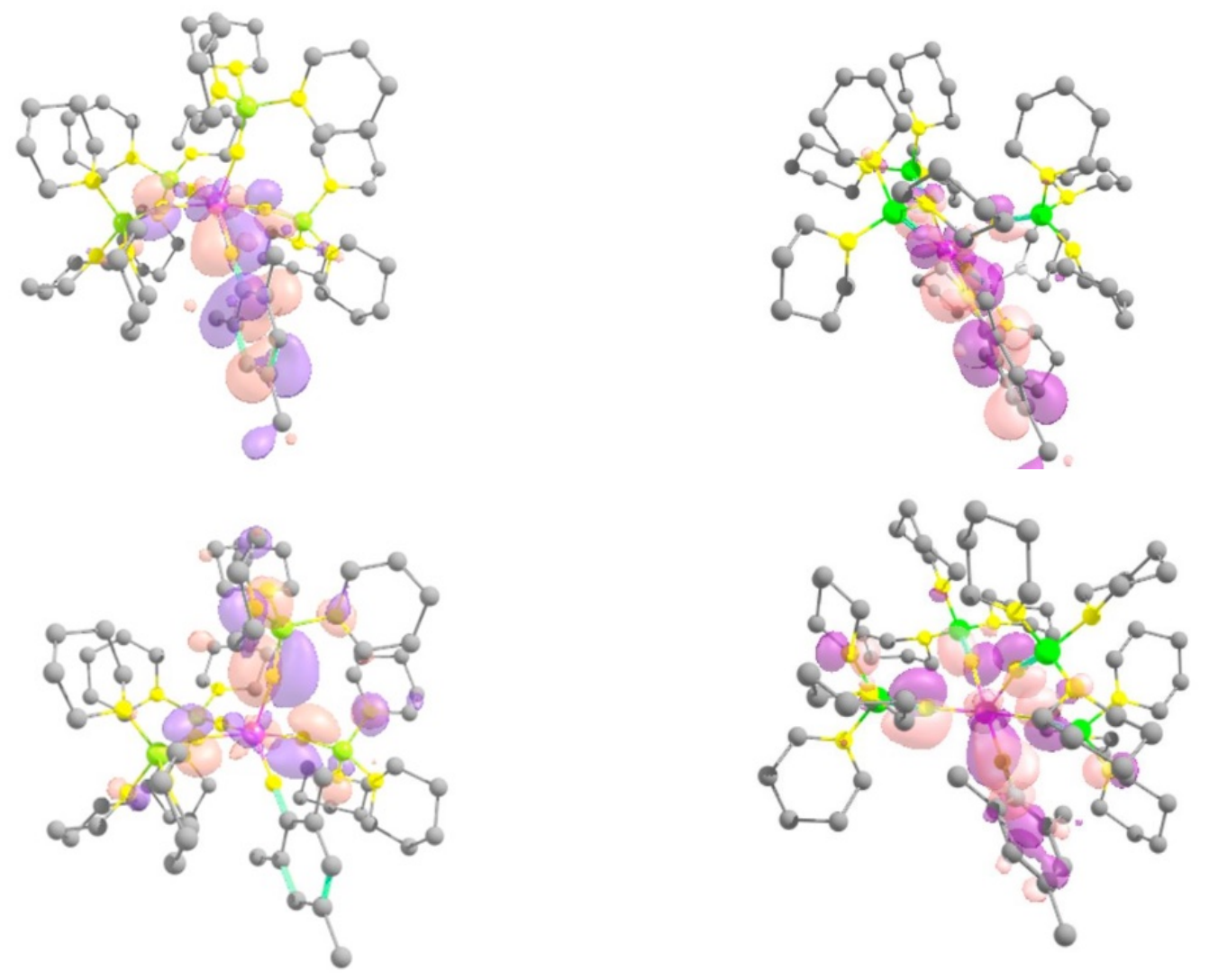

Figure S35. Clockwise from top left: The HOMO of the optimized geometry of 2U(PN)NMes. The HOMO of the forced geometry. The HOMO-1 of both optimized and square pyramidal. 
From the previous conclusions, the $p$ interactions between the NMes group was the driving force to stabilize the molecule. In this case an N-Methyl Imido group is placed instead of the NMes group.

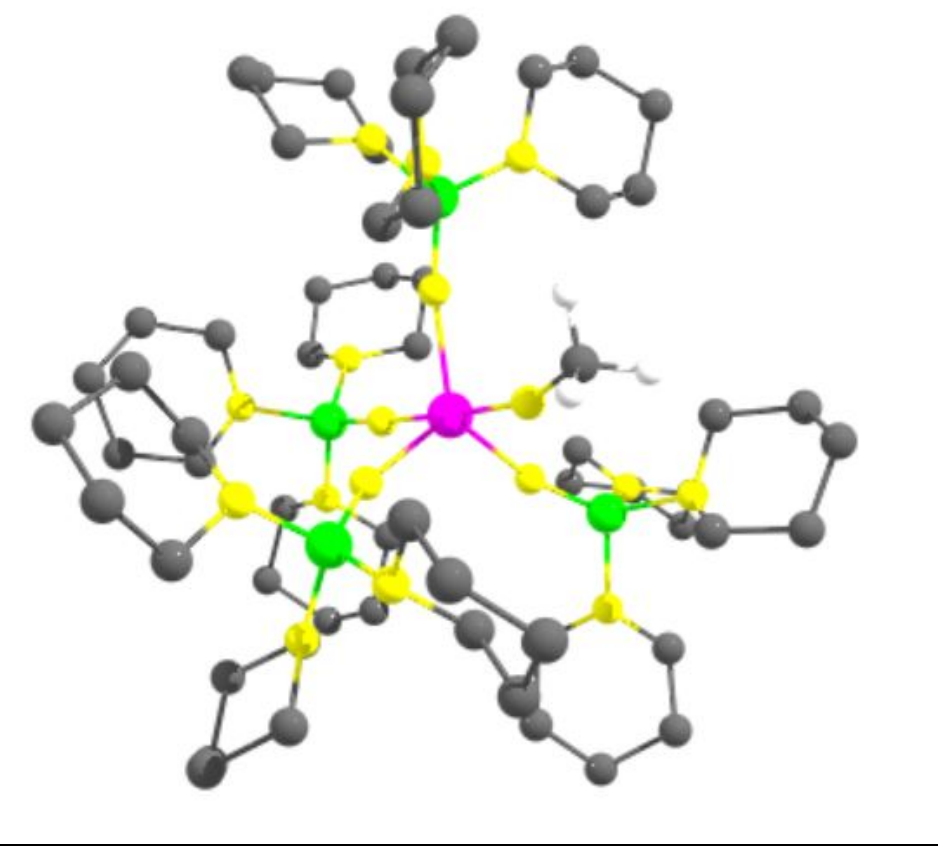

Figure S36. The optimized geometry of the newly substituted N-Methyl group in 2$\mathrm{U}(\mathrm{PN}) \mathbf{N}\left(\mathrm{CH}_{3}\right)$.

Table S28. The Wiberg Indexes (WBI) and bond distances and angles for 2$\mathrm{U}(\mathrm{PN}) \mathbf{N}\left(\mathrm{CH}_{3}\right)$.

\begin{tabular}{|c|c|c|c|c|}
\hline Bonding Coordinate & WBI & Bond Distance & $\begin{array}{c}\text { Bond } \\
\text { Coordinate }\end{array}$ & Bond Angles \\
\hline$U_{9}-N_{30}$ & 2.12 & 1.90 & $\begin{array}{c}\text { U9-N30- } \\
\text { C31 }\end{array}$ & 160.5 \\
\hline$N_{30}-C_{31}$ & 1.02 & 1.43 & $\mathrm{U} 9-\mathrm{N} 8-\mathrm{P} 7$ & 153.6 \\
\hline $\mathrm{U}_{9}-\mathrm{N}_{8}$ & 1.11 & 2.19 & $\begin{array}{c}\text { U9-N40- } \\
\text { P41 }\end{array}$ & 158.4 \\
\hline$U_{9}-N_{40}$ & 1.13 & 2.17 & $\begin{array}{c}\text { U9-N32- } \\
\text { P33 }\end{array}$ & 172.7 \\
\hline$U_{9}-N_{32}$ & 1.26 & 2.10 & $\begin{array}{c}\text { U9-N10- } \\
\text { P11 }\end{array}$ & 165.9 \\
\hline$U_{9}-N_{10}$ & 1.13 & 2.17 & $\begin{array}{l}\text { N30-U9- } \\
\text { N10 }\end{array}$ & 85.5 \\
\hline$N_{10}-P_{11}$ & 1.24 & 1.58 & $\begin{array}{c}\text { N30-U9- } \\
\text { N32 }\end{array}$ & 174.2 \\
\hline$N_{8}-P_{7}$ & 1.24 & 1.58 & $\begin{array}{l}\text { N30-U9- } \\
\text { N40 }\end{array}$ & 93.32 \\
\hline
\end{tabular}




\begin{tabular}{|c|c|c|c|c|}
\hline $\mathrm{N}_{40}-\mathrm{P}_{41}$ & 1.23 & 1.58 & N10-U9-N40 & 117.2 \\
\hline $\mathrm{N}_{32}-\mathrm{P}_{33}$ & 1.17 & 1.58 & N32-U9-N10 & 92.14 \\
\hline & & & N32-U9-N8 & 87.14 \\
\hline & & & N32-U9-N40 & 92.51 \\
\hline & & & N40-U9-N8 & 117.19 \\
\hline & & & N8-U9-N30 & 90.33 \\
\hline & & & N8-U9-N10 & 130.39 \\
\hline
\end{tabular}

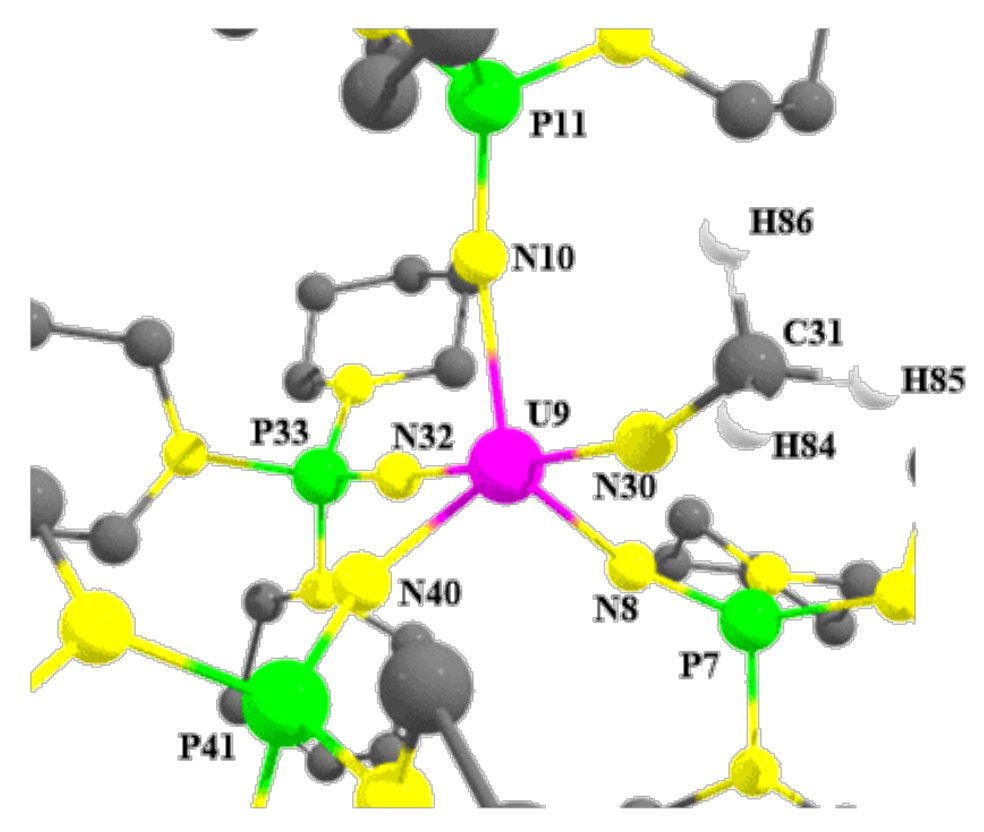

Figure S37. Optimized structure of hypothetical 2-U(PN)N(CH$\left.{ }_{3}\right)$ with atom labels.

Table S29. Contributions to U9-N8 bond in 2-U(PN)N( $\left.\mathrm{CH}_{3}\right)$.

\begin{tabular}{|c|c|c|c|c|c|c|c|c|}
\hline & \multicolumn{4}{|c|}{$U(9)$} & \multicolumn{4}{|c|}{$N(8)$} \\
\hline $\begin{array}{c}1^{\text {st }} \\
\text { Bond }\end{array}$ & \multicolumn{4}{|c|}{$12.37 \%$} & \multicolumn{4}{|c|}{$87.63 \%$} \\
\hline & $\mathrm{s}$ & $p$ & d & $\mathrm{f}$ & $\mathrm{s}$ & $p$ & $d$ & $\mathrm{f}$ \\
\hline & $2.76 \%$ & $20.39 \%$ & $27.63 \%$ & $49.15 \%$ & $4.36 \%$ & $95.63 \%$ & $0.02 \%$ & $0.00 \%$ \\
\hline $\begin{array}{l}2^{\text {nd }} \\
\text { Bond }\end{array}$ & \multicolumn{4}{|c|}{$12.38 \%$} & \multicolumn{4}{|c|}{$87.62 \%$} \\
\hline & $\mathrm{s}$ & $p$ & $d$ & $f$ & $\mathrm{~s}$ & $p$ & $d$ & $f$ \\
\hline & $0.71 \%$ & $21.62 \%$ & $30.86 \%$ & $46.74 \%$ & $3.04 \%$ & $96.94 \%$ & $0.02 \%$ & $0.00 \%$ \\
\hline
\end{tabular}


Table S30. Contributions to U9-N10 bond in $2-\mathbf{U}(\mathrm{PN}) \mathbf{N}\left(\mathrm{CH}_{3}\right)$.

\begin{tabular}{|c|c|c|c|c|c|c|c|c|}
\hline & \multicolumn{5}{|c|}{$\mathrm{U}(9)$} & \multicolumn{5}{c|}{$\mathrm{N}(10)$} \\
\hline $\begin{array}{c}1^{\text {st }} \\
\text { Bond }\end{array}$ & \multicolumn{5}{|c|}{$13.97 \%$} & \multicolumn{5}{c|}{$8.03 \%$} \\
\hline & $\mathrm{s}$ & $\mathrm{p}$ & $\mathrm{d}$ & $\mathrm{f}$ & $\mathrm{s}$ & $\mathrm{p}$ & $\mathrm{d}$ & $\mathrm{f}$ \\
\hline & $11.58 \%$ & $18.82 \%$ & $40.64 \%$ & $28.87 \%$ & $62.60 \%$ & $37.40 \%$ & $0.00 \%$ & $0.00 \%$ \\
\hline $\begin{array}{c}2^{\text {nd }} \\
\text { Bond }\end{array}$ & \multicolumn{7}{|c|}{$12.27 \%$} & \multicolumn{5}{c|}{$87.73 \%$} \\
\hline & $\mathrm{s}$ & $\mathrm{p}$ & $\mathrm{d}$ & $\mathrm{f}$ & $\mathrm{s}$ & $\mathrm{p}$ & $\mathrm{d}$ & $\mathrm{f}$ \\
\hline & $0.64 \%$ & $21.90 \%$ & $33.91 \%$ & $43.48 \%$ & $0.03 \%$ & $99.96 \%$ & $0.01 \%$ & $0.00 \%$ \\
\hline $\begin{array}{c}3^{\text {rd }} \\
\text { Bond }\end{array}$ & \multicolumn{2}{|c|}{$10.41 \%$} & \multicolumn{5}{|c|}{$89.59 \%$} & \\
\hline & $\mathrm{s}$ & $\mathrm{p}$ & $\mathrm{d}$ & $\mathrm{f}$ & $\mathrm{s}$ & $\mathrm{p}$ & $\mathrm{d}$ & $\mathrm{f}$ \\
\hline & $0.10 \%$ & $18.28 \%$ & $16.01 \%$ & $65.54 \%$ & $0.05 \%$ & $99.93 \%$ & $0.02 \%$ & $0.00 \%$ \\
\hline
\end{tabular}

Table S31. Contributions to U9-N30 bond in 2-U(PN)N(CH$)$.

\begin{tabular}{|c|c|c|c|c|c|c|c|c|}
\hline & \multicolumn{4}{|c|}{$U(9)$} & \multicolumn{4}{|c|}{$\mathrm{N}(30)$} \\
\hline $\begin{array}{c}1^{\text {st }} \\
\text { Bond }\end{array}$ & \multicolumn{4}{|c|}{$20.09 \%$} & \multicolumn{4}{|c|}{$79.91 \%$} \\
\hline & $\mathrm{s}$ & $p$ & d & $f$ & $\mathrm{~s}$ & $\mathrm{p}$ & $d$ & $f$ \\
\hline & $9.52 \%$ & $13.10 \%$ & $39.73 \%$ & $37.54 \%$ & $58.84 \%$ & $41.16 \%$ & $0.01 \%$ & $0.00 \%$ \\
\hline $\begin{array}{l}2^{\text {nd }} \\
\text { Bond }\end{array}$ & \multicolumn{4}{|c|}{$27.37 \%$} & \multicolumn{4}{|c|}{$72.63 \%$} \\
\hline & $\mathrm{s}$ & $p$ & $d$ & $f$ & $\mathrm{~s}$ & $p$ & $d$ & $f$ \\
\hline & $0.41 \%$ & $7.29 \%$ & $42.45 \%$ & $49.80 \%$ & $0.02 \%$ & $99.97 \%$ & $0.01 \%$ & $0.00 \%$ \\
\hline $\begin{array}{c}3^{\text {rd }} \\
\text { Bond }\end{array}$ & \multicolumn{4}{|c|}{$26.66 \%$} & \multicolumn{4}{|c|}{$73.34 \%$} \\
\hline & $\mathrm{s}$ & $\mathrm{p}$ & $\mathrm{d}$ & $f$ & $\mathrm{~s}$ & $p$ & $d$ & $f$ \\
\hline & $0.27 \%$ & $6.14 \%$ & $42.72 \%$ & $50.81 \%$ & $0.03 \%$ & $99.96 \%$ & $0.01 \%$ & $0.00 \%$ \\
\hline
\end{tabular}

Table S32. Contributions to N30-C31 bond in 2-U(PN)N(CH$)$.

\begin{tabular}{|c|c|c|c|c|c|c|c|c|}
\hline & \multicolumn{4}{|c|}{$\mathrm{N}(30)$} & \multicolumn{4}{c|}{$\mathrm{C}(31)$} \\
\hline $\begin{array}{c}1^{\text {st }} \\
\text { Bond }\end{array}$ & \multicolumn{4}{|c|}{$58.95 \%$} & \multicolumn{4}{c|}{$41.05 \%$} \\
\hline & $\mathrm{s}$ & $\mathrm{p}$ & $\mathrm{d}$ & $\mathrm{f}$ & $\mathrm{s}$ & $\mathrm{p}$ & $\mathrm{d}$ & $\mathrm{f}$ \\
\hline & $41.00 \%$ & $58.96 \%$ & $0.04 \%$ & $0.00 \%$ & $24.53 \%$ & $75.34 \%$ & $0.13 \%$ & $0.00 \%$ \\
\hline
\end{tabular}

Table S33. Contributions to U9-N32 bond in 2-U(PN)N(CH$)$.

\begin{tabular}{|c|c|c|c|c|c|c|c|c|}
\hline \multirow{3}{*}{$\begin{array}{c}1^{\text {st }} \\
\text { Bond }\end{array}$} & \multicolumn{4}{|c|}{$U(9)$} & \multicolumn{4}{|c|}{$\mathrm{N}(32)$} \\
\hline & \multicolumn{4}{|c|}{$16.71 \%$} & \multicolumn{4}{|c|}{$83.29 \%$} \\
\hline & $\mathrm{s}$ & $p$ & $d$ & $f$ & $\mathrm{~s}$ & $p$ & $d$ & $f$ \\
\hline
\end{tabular}




\begin{tabular}{|r|c|c|c|c|c|c|c|c|}
\hline & $9.18 \%$ & $15.69 \%$ & $38.30 \%$ & $36.72 \%$ & $60.26 \%$ & $39.73 \%$ & $0.00 \%$ & $0.00 \%$ \\
\hline $\begin{array}{c}2^{\text {nd }} \\
\text { Bond }\end{array}$ & \multicolumn{7}{|c|}{$12.20 \%$} & \multicolumn{5}{c|}{$87.80 \%$} \\
\hline & $\mathrm{s}$ & $\mathrm{p}$ & $\mathrm{d}$ & $\mathrm{f}$ & $\mathrm{s}$ & $\mathrm{p}$ & $\mathrm{d}$ & $\mathrm{f}$ \\
\hline & $0.06 \%$ & $10.42 \%$ & $38.25 \%$ & $51.19 \%$ & $0.00 \%$ & $99.99 \%$ & $0.01 \%$ & $0.00 \%$ \\
\hline $\begin{array}{c}3^{\text {rd }} \\
\text { Bond }\end{array}$ & \multicolumn{7}{|c|}{$11.36 \%$} & \multicolumn{5}{|c|}{$88.64 \%$} \\
\hline & $\mathrm{s}$ & $\mathrm{p}$ & $\mathrm{d}$ & $\mathrm{f}$ & $\mathrm{s}$ & $\mathrm{p}$ & $\mathrm{d}$ & $\mathrm{f}$ \\
\hline & $0.23 \%$ & $12.69 \%$ & $35.91 \%$ & $51.06 \%$ & $0.04 \%$ & $99.95 \%$ & $0.01 \%$ & $0.00 \%$ \\
\hline
\end{tabular}

Table S34. Contributions to U9-N40 bond in 2-U(PN)N(CH$)$.

\begin{tabular}{|c|c|c|c|c|c|c|c|c|}
\hline & \multicolumn{4}{|c|}{$U(9)$} & \multicolumn{4}{|c|}{$\mathrm{N}(40)$} \\
\hline $1^{\text {st }}$ & \multicolumn{4}{|c|}{$13.72 \%$} & \multicolumn{4}{|c|}{$86.28 \%$} \\
\hline & $\mathrm{s}$ & $p$ & $\mathrm{~d}$ & $f$ & $\mathrm{~s}$ & $p$ & $d$ & $f$ \\
\hline & $14.65 \%$ & $23.42 \%$ & $41.77 \%$ & $20.04 \%$ & $62.87 \%$ & $37.13 \%$ & $0.00 \%$ & $0.00 \%$ \\
\hline $\begin{array}{l}2^{\text {nd }} \\
\text { Bond }\end{array}$ & \multicolumn{4}{|c|}{$12.52 \%$} & \multicolumn{4}{|c|}{$87.48 \%$} \\
\hline & $\mathrm{s}$ & $p$ & $d$ & $f$ & $\mathrm{~s}$ & $p$ & d & $f$ \\
\hline & $0.15 \%$ & $19.04 \%$ & $38.70 \%$ & $42.05 \%$ & $0.02 \%$ & $99.97 \%$ & $0.02 \%$ & $0.00 \%$ \\
\hline $\begin{array}{c}3^{\text {rd }} \\
\text { Bond }\end{array}$ & \multicolumn{4}{|c|}{$10.45 \%$} & \multicolumn{4}{|c|}{$89.55 \%$} \\
\hline & $s$ & $p$ & $\mathrm{~d}$ & $f$ & $\mathrm{~s}$ & $p$ & $\mathrm{~d}$ & $f$ \\
\hline & $0.40 \%$ & $17.22 \%$ & $18.57 \%$ & $63.77 \%$ & $0.19 \%$ & $99.80 \%$ & $0.02 \%$ & $0.00 \%$ \\
\hline
\end{tabular}

With regards to the lone pairs present on the system, according to the relevant atoms shown in this study, the nitrogen(8) atoms is the only lone pair observed. In this instance it occupies an hybrid s/p orbital. When examining the contributions of electron density about the complex, this lone pair donates $\sim 53$ a.u. of density to the valence $p / f$ orbitals of the $U$ atom.

Overall, the interaction of the $\mathrm{N}$-methyl group to the $\mathrm{U}$ metal is of a double bond nature. The complex itself presents a highly delocalized environment with respect to the surrounding atoms on the $U$ atom. The bond length of the $U-N m e t h y l$ is the shortest and holds the strongest bonding character relative to the other ligands. This is an observation seen when the N-Mesityl ligand is coordinated to the metal center. 


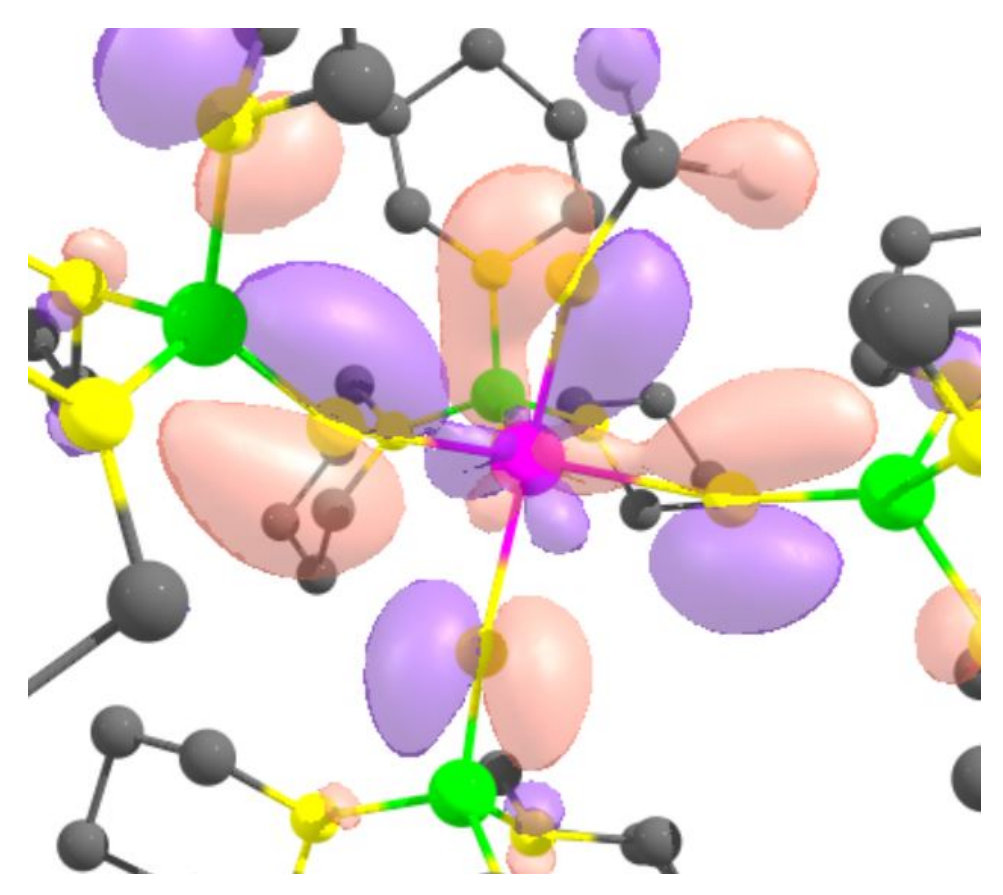

Figure S38. The HOMO for the U-Nmethyl complex and from left to right below: The $p$ interaction along with the 'third bond' where it is largely an interaction with adjacent ligands to the metal.

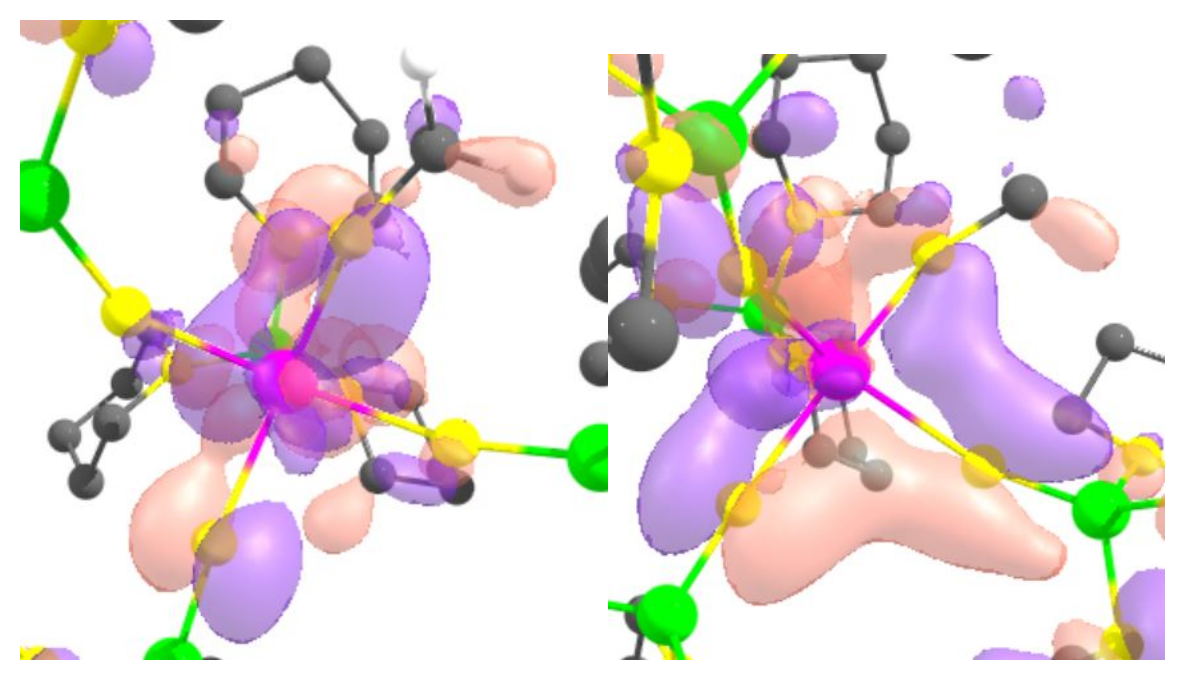

Figure S39. From left to right: the $p$ interaction along with the 'third bond' where it is largely an interaction with adjacent ligands to the metal. 


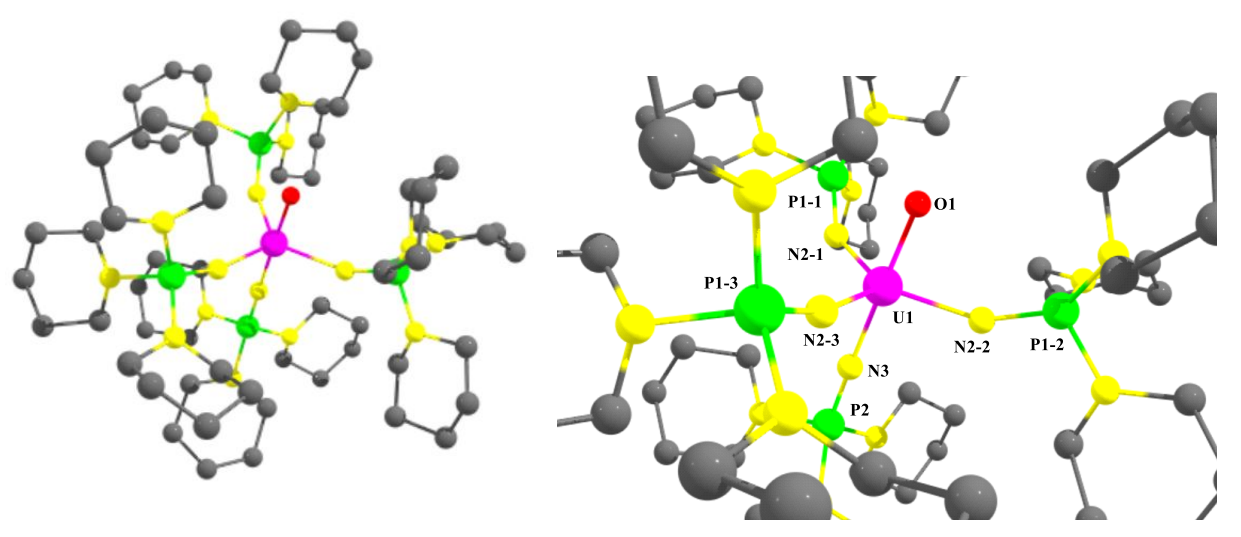

Figure S40. The optimized geometry of 2-U(PN)O.

Table S35. The Wiberg Indexes (WBI) and bond distances for 2-U(PN)O relative to the bond coordinates shown on the left.

\begin{tabular}{|c|c|c|}
\hline Bonding Coordinate & WBI & Bond Distance \\
\hline$U_{1}-\mathrm{O}_{1}$ & 2.1 & 1.81 \\
\hline$U_{1}-\mathrm{N}_{2-1}$ & 1.1 & 2.19 \\
\hline $\mathrm{U}_{1}-\mathrm{N}_{2-2}$ & 1.1 & 2.19 \\
\hline $\mathrm{U}_{1}-\mathrm{N}_{2-3}$ & 1.1 & 2.18 \\
\hline $\mathrm{U}_{1}-\mathrm{N}$ & 1.3 & 2.05 \\
\hline $\mathrm{N}_{2-1}-\mathrm{P}_{1-1}$ & 1.2 & 1.58 \\
\hline $\mathrm{N}_{2-2}-\mathrm{P}_{1-2}$ & 1.2 & 1.58 \\
\hline $\mathrm{N}_{2-3}-\mathrm{P}_{1-3}$ & 1.2 & 1.58 \\
\hline $\mathrm{N}_{3}-\mathrm{P}_{2}$ & 1.1 & 1.59 \\
\hline
\end{tabular}

Table S36. Contributions to U1-O1 bond in 2-U(PN)O.

\begin{tabular}{|c|c|c|c|c|c|c|c|c|}
\hline & \multicolumn{4}{|c|}{$\mathrm{U}(1)$} & \multicolumn{4}{|c|}{$\mathrm{O}(1)$} \\
\hline $\begin{array}{c}1^{\text {st }} \\
\text { Bond }\end{array}$ & \multicolumn{4}{|c|}{$19.29 \%$} & \multicolumn{4}{|c|}{$80.71 \%$} \\
\hline & $s$ & $p$ & $d$ & f & $s$ & $p$ & $d$ & $f$ \\
\hline & $0.00 \%$ & $8.59 \%$ & $45.42 \%$ & $45.87 \%$ & $0.00 \%$ & $99.84 \%$ & $0.16 \%$ & $0.00 \%$ \\
\hline $\begin{array}{l}2^{\text {nd }} \\
\text { Bond }\end{array}$ & \multicolumn{4}{|c|}{$19.43 \%$} & \multicolumn{4}{|c|}{$80.57 \%$} \\
\hline & $s$ & $p$ & $\mathrm{~d}$ & $f$ & $\mathrm{~s}$ & $p$ & $d$ & $f$ \\
\hline & $0.04 \%$ & $8.95 \%$ & $44.80 \%$ & $46.09 \%$ & $0.53 \%$ & $99.32 \%$ & $0.16 \%$ & $0.00 \%$ \\
\hline $\begin{array}{l}3^{\text {rd }} \\
\text { Bond }\end{array}$ & \multicolumn{4}{|c|}{$22.22 \%$} & \multicolumn{4}{|c|}{$77.78 \%$} \\
\hline & $s$ & $p$ & $\mathrm{~d}$ & $f$ & $s$ & $p$ & d & $f$ \\
\hline
\end{tabular}


Table S37. Contributions to U1-N2(1) bond in 2-U(PN)O.

\begin{tabular}{|c|c|c|c|c|c|c|c|c|}
\hline & \multicolumn{4}{|c|}{$U(1)$} & \multicolumn{4}{|c|}{ N2-(1) } \\
\hline $\begin{array}{c}1^{\text {st }} \\
\text { Bond }\end{array}$ & \multicolumn{4}{|c|}{$13.17 \%$} & \multicolumn{4}{|c|}{$86.83 \%$} \\
\hline & $s$ & $\mathrm{p}$ & d & $f$ & $\mathrm{~s}$ & $\mathrm{p}$ & d & $f$ \\
\hline & $7.24 \%$ & $20.86 \%$ & $42.91 \%$ & $28.90 \%$ & $62.40 \%$ & $37.60 \%$ & $0.01 \%$ & $0.00 \%$ \\
\hline $\begin{array}{l}2^{\text {nd }} \\
\text { Bond }\end{array}$ & \multicolumn{4}{|c|}{$12.75 \%$} & \multicolumn{4}{|c|}{$87.25 \%$} \\
\hline & $\mathrm{s}$ & $\mathrm{p}$ & $\mathrm{d}$ & $f$ & $\mathrm{~s}$ & $\mathrm{p}$ & d & $f$ \\
\hline & $0.01 \%$ & $27.60 \%$ & $30.92 \%$ & $41.41 \%$ & $0.02 \%$ & $99.95 \%$ & $0.03 \%$ & $0.00 \%$ \\
\hline $\begin{array}{l}3^{\text {rd }} \\
\text { Bond }\end{array}$ & \multicolumn{4}{|c|}{$9.15 \%$} & \multicolumn{4}{|c|}{$90.85 \%$} \\
\hline & $\mathrm{s}$ & $p$ & $\mathrm{~d}$ & $\mathrm{f}$ & $\mathrm{s}$ & $p$ & d & $\mathrm{f}$ \\
\hline & $0.26 \%$ & $11.52 \%$ & $15.71 \%$ & $72.44 \%$ & $0.64 \%$ & $99.33 \%$ & $0.03 \%$ & $0.00 \%$ \\
\hline
\end{tabular}

Table S38. Contributions to U1-N2(2) bond in 2-U(PN)O.

\begin{tabular}{|c|c|c|c|c|c|c|c|c|}
\hline & \multicolumn{4}{|c|}{$U(1)$} & \multicolumn{4}{|c|}{ N2-(2) } \\
\hline $\begin{array}{c}1^{\text {st }} \\
\text { Bond }\end{array}$ & \multicolumn{4}{|c|}{$13.05 \%$} & \multicolumn{4}{|c|}{$86.95 \%$} \\
\hline & $\mathrm{s}$ & $p$ & d & $f$ & $s$ & $\mathrm{p}$ & d & $f$ \\
\hline & $7.28 \%$ & $25.98 \%$ & $40.81 \%$ & $25.84 \%$ & $62.41 \%$ & $37.58 \%$ & $0.01 \%$ & $0.00 \%$ \\
\hline \multirow[t]{3}{*}{$\begin{array}{c}2^{\text {nd }} \\
\text { Bond }\end{array}$} & \multicolumn{4}{|c|}{$12.70 \%$} & \multicolumn{4}{|c|}{$87.30 \%$} \\
\hline & $\mathrm{s}$ & $p$ & d & $f$ & $s$ & $p$ & $d$ & $f$ \\
\hline & $0.01 \%$ & $28.11 \%$ & $30.80 \%$ & $41.01 \%$ & $0.01 \%$ & $99.97 \%$ & $0.02 \%$ & $0.00 \%$ \\
\hline $\begin{array}{c}3^{\text {rd }} \\
\text { Bond }\end{array}$ & \multicolumn{4}{|c|}{$9.45 \%$} & \multicolumn{4}{|c|}{$90.55 \%$} \\
\hline & $\mathrm{s}$ & $\mathrm{p}$ & d & $f$ & $\mathrm{~s}$ & $\mathrm{p}$ & d & $f$ \\
\hline & $0.20 \%$ & $9.13 \%$ & $16.91 \%$ & $73.68 \%$ & $0.56 \%$ & $99.41 \%$ & $0.03 \%$ & $0.00 \%$ \\
\hline
\end{tabular}


Table S39. Contributions to U1-N2(3) bond in 2-U(PN)O.

\begin{tabular}{|c|c|c|c|c|c|c|c|c|}
\hline & \multicolumn{4}{|c|}{$\mathrm{U}(1)$} & \multicolumn{4}{|c|}{ N2-(3) } \\
\hline $\begin{array}{c}1^{\text {st }} \\
\text { Bond }\end{array}$ & \multicolumn{4}{|c|}{$13.50 \%$} & \multicolumn{4}{|c|}{$86.50 \%$} \\
\hline & $\mathrm{s}$ & $p$ & d & $\mathrm{f}$ & $\mathrm{s}$ & $p$ & d & $f$ \\
\hline & $7.20 \%$ & $24.87 \%$ & $42.19 \%$ & $25.64 \%$ & $60.99 \%$ & $39.01 \%$ & $0.00 \%$ & $0.00 \%$ \\
\hline $\begin{array}{l}2^{\text {nd }} \\
\text { Bond }\end{array}$ & \multicolumn{4}{|c|}{$12.02 \%$} & \multicolumn{4}{|c|}{$87.98 \%$} \\
\hline & $\begin{array}{c}s \\
0.01 \%\end{array}$ & $\begin{array}{c}p \\
28.47 \%\end{array}$ & $\begin{array}{c}\mathrm{d} \\
29.93 \%\end{array}$ & $\begin{array}{c}f \\
41.51 \%\end{array}$ & $\begin{array}{c}s \\
0.04 \%\end{array}$ & $\begin{array}{c}p \\
99.95 \%\end{array}$ & $\begin{array}{c}d \\
0.01 \%\end{array}$ & $\begin{array}{c}f \\
0.00 \%\end{array}$ \\
\hline
\end{tabular}

Table S40. Contributions to U1-N3 bond in 2-U(PN)O.

\begin{tabular}{|c|c|c|c|c|c|c|c|c|}
\hline & \multicolumn{4}{|c|}{$U(1)$} & \multicolumn{4}{|c|}{$\mathrm{N}(3)$} \\
\hline $\begin{array}{l}1^{\text {st }} \\
\text { Bond }\end{array}$ & \multicolumn{4}{|c|}{$17.79 \%$} & \multicolumn{4}{|c|}{$82.21 \%$} \\
\hline & $s$ & $p$ & d & $f$ & $s$ & $p$ & d & $f$ \\
\hline & $10.85 \%$ & $9.38 \%$ & $39.99 \%$ & $39.62 \%$ & $58.56 \%$ & $41.43 \%$ & $0.01 \%$ & $0.00 \%$ \\
\hline $\begin{array}{l}2^{\text {nd }} \\
\text { Bond }\end{array}$ & \multicolumn{4}{|c|}{$12.61 \%$} & \multicolumn{4}{|c|}{$87.39 \%$} \\
\hline & s & $\begin{array}{c}p \\
1099 \%\end{array}$ & $\frac{d}{3844 \%}$ & $\frac{f}{5046 \%}$ & $s$ & $p$ & $\frac{d}{d}$ & $f$ \\
\hline $\begin{array}{l}3^{\text {rd }} \\
\text { Bond }\end{array}$ & \multicolumn{4}{|c|}{$12.80 \%$} & \multicolumn{4}{|c|}{$87.20 \%$} \\
\hline & $\begin{array}{c}\mathrm{s} \\
0.01 \%\end{array}$ & $\frac{p}{11.11 \%}$ & $\begin{array}{c}\mathrm{d} \\
39.39 \%\end{array}$ & $\frac{f}{49.41 \%}$ & $\begin{array}{c}\mathrm{s} \\
0.04 \%\end{array}$ & $\begin{array}{c}\mathrm{p} \\
99.96 \%\end{array}$ & $\begin{array}{c}\frac{d}{0.00 \%} \\
0.00\end{array}$ & $\begin{array}{c}\mathrm{f} \\
0.00 \%\end{array}$ \\
\hline
\end{tabular}



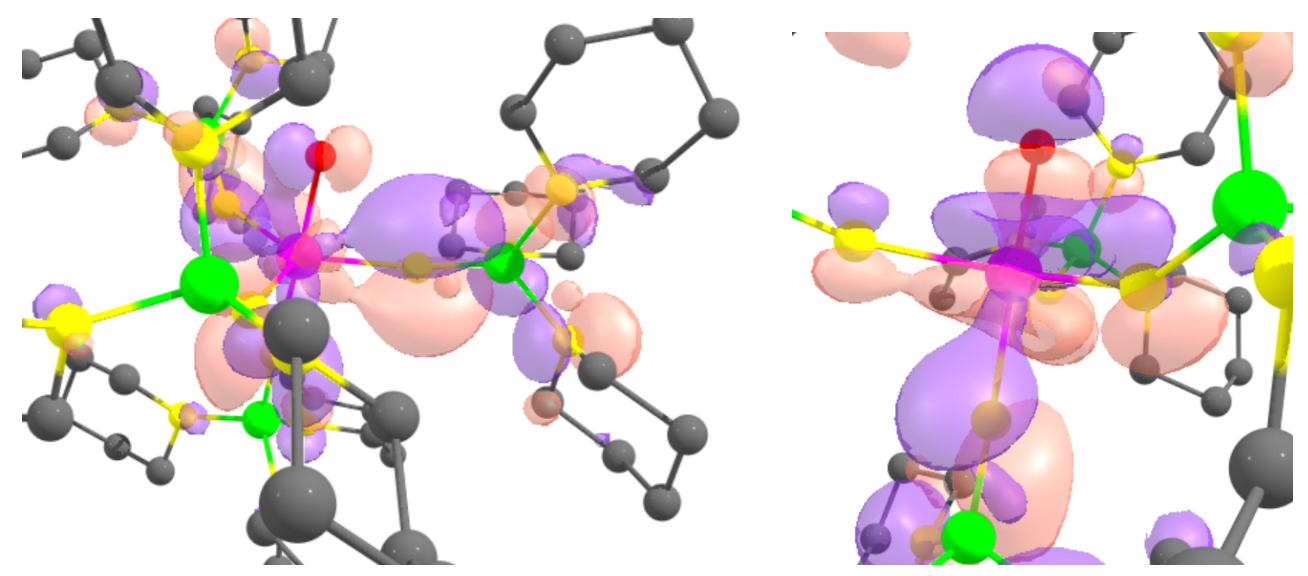

Figure S41. The above orbital image represents the $p$ interaction into the $d / f$ orbitals of the $U$ atom in 2-U(PN)O. In this instance, the coordinate in question is U1-N2(2). Furthermore, you can see the interaction of the lone pair on the oxygen atom towards the bonding between the $U$ atoms. This clearly shows the $\mathrm{P}-\mathrm{d}$ interaction from the lone pair as expected.

Table S41. Natural Population Analysis (NPA) for 2-U(PN)O.

\begin{tabular}{|c|c|}
\hline Atom & Natural Charge \\
\hline U1 & 1.5 \\
\hline O1 & -0.6 \\
\hline N2-1 & -1.3 \\
\hline P2-1 & 2.5 \\
\hline N2-2 & -1.3 \\
\hline P2-2 & 2.5 \\
\hline P2-3 & -1.3 \\
\hline N3 & 2.5 \\
\hline P3 & -1.2 \\
\hline
\end{tabular}

Table 42. Mulliken Charges for 2-U(PN)O.

\begin{tabular}{|c|c|}
\hline Atom & Mulliken Charge \\
\hline U1 & 1.5 \\
\hline O1 & -0.6 \\
\hline N2-1 & -0.6 \\
\hline P2-1 & 0.6 \\
\hline P2-2 & -0.6 \\
\hline N2-3 & 0.6 \\
\hline P2-3 & -0.6 \\
\hline N3 & 0.6 \\
\hline P3 & -0.5 \\
\hline & 0.6 \\
\hline
\end{tabular}


Table 43. Orbital $6 p$ data for $2-\mathrm{U}(\mathrm{PN}) \mathrm{O}$.

Occupancy Energy

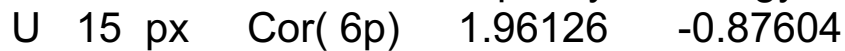

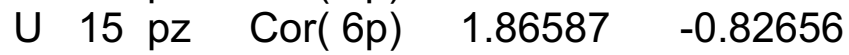

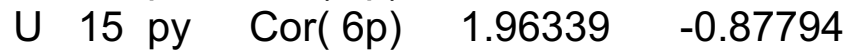




\section{References}

1. M. J. Monreal, R. K. Thomson, T. Cantat, N. E. Travia, B. L. Scott and J. L. Kiplinger, Organometallics, 2011, 30, 2031-2038.

2. N. T. Rice, J. Su, T. P. Gompa, D. R. Russo, J. Telser, L. Palatinus, J. Bacsa, P. Yang, E. R. Batista and H. S. La Pierre, Inorganic Chemistry, 2019, 58, 5289-5304.

3. S. A. Johnson, J. J. Kiernicki, P. E. Fanwick and S. C. Bart, Organometallics, 2015, 34, 2889-2895.

4. G. R. Fulmer, A. J. M. Miller, N. H. Sherden, H. E. Gottlieb, A. Nudelman, B. M. Stoltz, J. E. Bercaw and K. I. Goldberg, Organometallics, 2010, 29, 2176-2179.

5. G. Sheldrick, Acta Crystallographica Section A, 2015, 71, 3-8.

6. O. V. Dolomanov, L. J. Bourhis, R. J. Gildea, J. A. K. Howard and H. Puschmann, Journal of Applied Crystallography, 2009, 42, 339-341.

7. G. M. Sheldrick, Acta crystallographica. Section C, Structural chemistry, 2015, 71, 3-8.

8. A. D. Becke, The Journal of Chemical Physics, 1993, 98, 5648-5652.

9. J. P. Perdew and Y. Wang, Physical Review B, 1992, 45, 13244-13249.

10. Gaussian 09, Revision D.01, M. J. Frisch, G. W. Trucks, H. B. Schlegel, G. E. Scuseria, M. A. Robb, J. R. Cheeseman, G. Scalmani, V. Barone, G. A. Petersson, H. Nakatsuji, X. Li, M. Caricato, A. V. Marenich, J. Bloino, B. G. Janesko, R. Gomperts, B. Mennucci, H. P. Hratchian, J. V. Ortiz, A. F. Izmaylov, J. L. Sonnenberg, Williams, F. Ding, F. Lipparini, F. Egidi, J. Goings, B. Peng, A. Petrone, T. Henderson, D. Ranasinghe, V. G. Zakrzewski, J. Gao, N. Rega, G. Zheng, W. Liang, M. Hada, M. Ehara, K. Toyota, R.

Fukuda, J. Hasegawa, M. Ishida, T. Nakajima, Y. Honda, O. Kitao, H. Nakai, T. Vreven, K. Throssell, J. A. Montgomery Jr., J. E. Peralta, F. Ogliaro, M. J. Bearpark, J. J. Heyd, E. N. Brothers, K. N. Kudin, V. N. Staroverov, T. A. Keith, R. Kobayashi, J. Normand, K. Raghavachari, A. P. Rendell, J. C. Burant, S. S. Iyengar, J. Tomasi, M. Cossi, J. M. Millam, M. Klene, C. Adamo, R. Cammi, J. W. Ochterski, R. L. Martin, K. Morokuma, O. Farkas, J. B. Foresman and D. J. Fox, Gaussian, Inc., Wallingford Ct, 2009.

11. M. Dolg, U. Wedig, H. Stoll and H. Preuss, The Journal of Chemical Physics, 1987, 86, 866-872.

12. M. Dolg, Effective Core Potentials. Modern Methods and Algorithm of Quantum Chemistry, Vol. 1; Ed.: J. Grotendorst; John von Neuman Institute for Computing, Jülich, Germany, 2000; pp. 507.

13. D. Andrae, U. Häußermann, M. Dolg, H. Stoll and H. Preuß, Theoretica chimica acta, 1990, 77, 123-141.

14. A. W. Ehlers, M. Böhme, S. Dapprich, A. Gobbi, A. Höllwarth, V. Jonas, K. F. Köhler, R. Stegmann, A. Veldkamp and G. Frenking, Chemical Physics Letters, 1993, 208, 111 114.

15. W. Küchle, M. Dolg, H. Stoll and H. Preuss, The Journal of Chemical Physics, 1994, 100, 7535-7542.

16. X. Cao, M. Dolg and H. Stoll, The Journal of Chemical Physics, 2002, 118, 487-496.

17. X. Cao and M. Dolg, Journal of Molecular Structure: THEOCHEM, 2004, 673, 203-209.

18. A. Bergner, M. Dolg, W. Küchle, H. Stoll and H. Preuß, Molecular Physics, 1993, 80, 1431-1441.

19. P. C. Hariharan and J. A. Pople, Theoretica chimica acta, 1973, 28, 213-222.

20. A. E. Reed, L. A. Curtiss and F. Weinhold, Chemical Reviews, 1988, 88, 899-926. 